Review

\title{
Genus Stachys: A Review of Traditional Uses, Phytochemistry and Bioactivity
}

\author{
Ekaterina-Michaela Tomou, Christina Barda and Helen Skaltsa *
}

Department of Pharmacognosy and Chemistry of Natural Products, Faculty of Pharmacy, School of Health Sciences, National \& Kapodistrian University of Athens, Panepistimiopolis, Zografou, 15771 Athens, Greece; ktomou@pharm.uoa.gr (E.-M.T.); cbarda@pharm.uoa.gr (C.B.)

* Correspondence: skaltsa@pharm.uoa.gr; Tel.: +30-2107274593

Received: 11 August 2020; Accepted: 25 September 2020; Published: 29 September 2020

\begin{abstract}
Background: The genus Stachys L. (Lamiaceae) includes about 300 species as annual or perennial herbs or small shrubs, spread in temperate regions of Mediterranean, Asia, America and southern Africa. Several species of this genus are extensively used in various traditional medicines. They are consumed as herbal preparations for the treatment of stress, skin inflammations, gastrointestinal disorders, asthma and genital tumors. Previous studies have investigated the chemical constituents and the biological activities of these species. Thus, the present review compiles literature data on ethnomedicine, phytochemistry, pharmacological activities, clinical studies and the toxicity of genus Stachys. Methods: Comprehensive research of previously published literature was performed for studies on the traditional uses, bioactive compounds and pharmacological properties of the genus Stachys, using databases with different key search words. Results: This surey documented 60 Stachys species and 10 subspecies for their phytochemical profiles, including 254 chemical compounds and reported 19 species and 4 subspecies for their pharmacological properties. Furthermore, 25 species and 6 subspecies were found for their traditional uses. Conclusions: The present review highlights that Stachys spp. consist an important source of bioactive phytochemicals and exemplifies the uncharted territory of this genus for new research studies.
\end{abstract}

Keywords: Stachys L.; traditional uses; pharmacological activities; phytochemicals; bioactive compounds

\section{Introduction}

The genus Stachys L., a large member of the Lamiaceae family, comprises more than 300 species, dispersing in temperate and tropical regions of Mediterranean, Asia, America and southern Africa [1-3]. Up to now, the most established and comprehensive classification of the genus is introduced by Bhattacharjee (1980), categorizing into two subgenera Betonica L. and Stachys L. [2,3]. The subgenus Stachys includes 19 sections, while the subgenus Betonica comprises 2 sections [1]. However, the two subgenera present important botanical and phytochemical differences which differentiate them $[1,4,5]$.

Stachys species grow as annual or perennial herbs or small shrubs with simple petiolate or sessile leaves. The number of verticillate ranges from four to many-flowered, usually forming a terminal spike-like inflorescence. Calyx tubes are tubular-campanulate, 5 or 10 veined, regular or weakly bilabiate with five subequal teeth. Corolla has a narrow tube, 2-lipped; upper lip flat or hooded and generally hairy, while the lower lip is 3-lopped and glabrous to hairy. The nutlets are oblong to ovoid, rounded at apex [6].

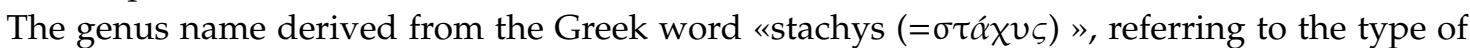
the inflorescence which is characterized as "spike of corn" and resembles to the inflorescences of the species of genus Triticum L. (Gramineae). In ancient times, the name "stachys" referred mainly to the 
species Stachys germanica L. whose inflorescence is like an ear and is covered with off-white trichome [7]. The Latin name of the genus is trifarium (=tomentose) [8].

Historically, Dioscorides mentioned the species S. germanica L. with the name "stachys" [9]. However, in late Byzantine era, 'Nikolaos Myrepsos' included some species of the genus Stachys (S. germanica L., S. officinalis (L.) Travis, S. alopecuros (L.) Benth.) in his medical manuscript "Dynameron". Precisely, S. officinalis and S. alopecuros were probably included in 11 recipes, under the names vetoniki, drosiovotanon, lauriole, kakambri, while S. germanica was added in 1 recipe referred as stachys [10].

Many species of the genus are extensively used in traditional medicine of several countries, having various names. For instance, the species $S$. recta, known as yellow woundwort, is called as "erba della paura" (="herb that keeps away fear") in Italy, attributing to the anxiolytic properties of its herbal tea, while S. lavandulifolia Vahl is called as "Chaaye Koohi" in Iran [11-13]. In addition, herbal preparations of Stachys spp. are widely consumed in folk medicine to treat a broad array of disorders and diseases, including stress, skin inflammations, stomach disorders and genital tumors $[3,14,15]$. Specially, the herbal teas of these plants, known as "mountain tea", are used for skin and stomach disorders $[12,16]$. The latter common name could lead to a misinterpretation since the herbal remedies of any Sideritis species are globally known with the same name.

In the international literature, Stachys species have been broadly studied through several phytochemical and pharmacological investigations, justifying their ethnopharmacological uses. Of special pharmacological interest are considered the anti-inflammatory, antioxidant, analgesic, renoprotective, anxiolytic and antidepressant activity [3,17-19]. The range of the therapeutic properties attributed to these species have been associated to their phytochemical content. Therefore, genus Stachys has received much attention for the screening of its bioactive secondary metabolites from different plant parts. In general, more than 200 compounds have been isolated from this genus, belonging to the following important chemical groups; terpenes (e.g., triterpenes, diterpenes, iridoids), polyphenols (e.g., flavone derivatives, phenylethanoid glycosides, lignans), phenolic acids and essential oils [3,5,14,20-22].

Consequently, plants of genus Stachys are considered a great source of phytochemicals with therapeutic and economic applications. Given the increasing demand for natural products, many Stachys species have been cultivated for uses in traditional medicine, in food market, in cosmetic industry and for ornamental reasons [21,22]. Despite the widely uses of the specific species and the large amount of research studies, there has been no recent comprehensive review including all the latest data of the specific genus and its contribution in medicine. Up to now, the available reviews are centered to the phytochemical profile and biological activities of Stachys spp. in correlation to chemotaxonomy approach [3,21-23]. Thus, this review summarizes the current state of knowledge on the traditional uses, phytochemistry, pharmacological activities, clinical studies and toxicity of the genus Stachys L.

\section{Materials and Methods}

A comprehensive search on previous studies was conducted on scientific databases such as PubMed, Scopus, Google scholar and Reaxys, including the years 1969-2020. The search terms "Stachys", "Stachys compounds", "Stachys phytochemicals", "Stachys pharmacological" and "Stachys traditional uses" were used for data collection. Searches were performed for other potential studies by manual screening references in the identified studies. In total, 161 publications describing the traditional uses, bioactive compounds, pharmacological properties and the toxicity of the genus Stachys were included, excluding articles focuses on taxonomy, botany and agronomy. The traditional medicinal uses of Stachys species were reported in Table 1, while the isolated specialized products were categorized by species in Tables 2-15, with the attempt of the discrimination between publications describing metabolites' isolation (including NMR data) or identification/screening (by means of HPLC, LC-MS, etc.). The chemical structures of the bioactive compounds were showed in Tables 16-29. The reported biological activities of extracts/compounds of the last five years were mentioned by Stachys species in Table 30. The general characteristics of the analyzed studies in the current review 
are showed in Table 31. According to recent publications which support the division of the genus Stachys based on Bhattacharjee (1980), the classification in the present review is formed on this latter study. The species name and their synonyms are quoted as reporting in databases "Plant list" or "Euro + Med" or "IPNI" [24-26].

\section{Traditional Medicinal Uses of Genus Stachys}

Several Stachys spp. have been used in various ethnomedicines for thousands of years. A plethora studies mentioned their diverse traditional medicinal uses. In the current review, a detailed description of the available data of the traditional uses of Stachys spp. is shown in Table 1, reporting 25 species and 6 subspecies of this genus. A careful overview of the specific table reveals that the ethnomedicinal use of Stachys spp. is particularly in the area covering of Mediterannean to Iran. Most of the species are consumed as herbal teas for the treatment of infections, common cold, gastrointestinal disorders, inflammation, skin disorders/wounds, asthma and anxiety.

The species $S$. affinis is widely used in Chinese traditional medicine for several uses such as common cold, heart disease, pain relief, antioxidant activity, ischemic brain injury, dementia and gastrointestinal related diseases [27-30]. Another species applied in Chinese folk medicine is S. geobombycis, known as DongChongXiaCao, which is recommended as tonic and interestingly, this species is also used in Europe and Japan [22].

In Iran, several species are applied as traditional therapeutic agents in various conditions, including S. acerosa [31], S. fruticulosa [32], S. byzantina (known in Farsi as "lamb's ear" or "lamb's tongue" or "sonbolehe noghrehi" or "zabanehe bare") [33-35], S. inflata (local names; "poulk" or "Ghol-e-Argavan") [31,36,37], S. lavandulifolia (known as "Chaaye Koohi") [12,13,31,38-44], S. pilifera [31,45], S. schtschegleevii [32,34,46], S. sylvatica [47] and S. turcomanica [34]. Of considerable interest, S. sylvatica (common name "hedge woundwort") is recommended for the treatment of women with polycystic ovary syndrome (PCOS) [47].

Furthermore, in Turkish folk medicine, the species S. cretica subsp. anatolica, S. cretica subsp. mersinaea, S. iberica subsp. georgica, S. iberica subsp. stenostachya, S. kurdica, S. lavandulifolia and S. obliqua are used mainly to treat colds, cough, stomach ache and as antipyretic agents, while S. sylvatica is applied in cardiac disorders [22,48-50].

In Italy, the infusions of the leaves of $S$. annua and S. recta are used to wash the face to reveal headache [51], whereas the aerial parts of the subspecies S. annua subsp. annua, known as "stregona annual" or "erba strega", are consumed as anti-catarrhal, febrifuge, tonic and vulnerary [52]. The decoction of the aerial parts of $S$. recta is also consumed as purative and for bad luck/spirit $[53,54]$. Interestingly, S. annua and S. arvensis, as well as the subspecies $S$. recta subsp. recta are applied against evil eye $[11,51,52,55]$. Moreover, in an area of central Italy, the species $S$. officinalis is used as oily extract to treat wounds and to dye wood yellow $[29,54]$. To be mentioned that $S$. recta is listed in the European Pharmacopeia, as well as S. officnalis is mentioned in Anthroposophic Pharmaceutical Codex (APC) [22]. However, Gören (2011) reported that some species (e.g., S. annua, S. recta and S. sylvatica) have been mentioned to be poisonous [22].

In North Greece, the infusion and decoction of $S$. iva are consumed against common cold and gastrointestinal disorders [56]. In addition, Fazio et al. (1994) reported different formulations of the Greek species S. mucronata applied in Greek tradition medicine. Precisely, the decoction of this species is consumed as an antirheumatic and antineuralgic agent, as well as the juice of fresh leaves is applied in wounds and ulcers. Moreover, the infusion of fresh leaves has antidiarrhoic effect, while the infusion of roots is purgative [57].

In addition to traditional medicinal uses, some species of genus Stachys are also consumed as edible plants, vegetables and food additives like the tubers of S. affinis (known as Chinese artichoke/chorogi; China/Japan) in China and Japan [22,27], the aerial parts of S. lavandulifolia in Iran [31], or the S. palustris in Poland [22,58]. The latter species is also included in the diet in Sweden, Ukraine and Great Britain [22]. Moreover, the dried powder of S. palustris is used as an additive for bread in Europe, thus it is known as "mayday flour" [22]. 
The infusion of the aerial parts of Stachys sp. LAM is used as traditional remedy for colic, gases and swollen stomach in Peru [22,59]. It is noteworthy to mention that a few species have been used in veterinary such as $S$. germanica and S. officinalis [30,54]. 
Table 1. Stachys species with reported traditional medicinal uses.

\begin{tabular}{|c|c|c|c|c|}
\hline Species & $\begin{array}{l}\text { Geographical Origin of } \\
\text { the Reported } \\
\text { Traditional Use }\end{array}$ & Traditional Medicinal Use & $\begin{array}{l}\text { Preparation and/or } \\
\text { Administration/ } \\
\text { Parts of the Plant }\end{array}$ & Ref. \\
\hline S. acerosa Boiss. & Iran & Common cold & Decoction & [31] \\
\hline \multirow{2}{*}{ S. affinis Bunge (=S. sieboldii Miq.) } & China & $\begin{array}{c}\text { Infections, colds, heart } \\
\text { diseases, tuberculosis, pneumonia }\end{array}$ & Edible food (tubers) & {$[27,28]$} \\
\hline & China & $\begin{array}{l}\text { Common cold, heart diseases, for pain relief, as antioxidant, } \\
\text { to treat ischemic brain injury, dementia, various gastrointestinal } \\
\text { related diseases }\end{array}$ & - & [29] \\
\hline S. annua (L.) L & Italy & Headache & $\begin{array}{c}\text { Infusion of leaves; also, external } \\
\text { use to wash face }\end{array}$ & [51] \\
\hline S. annua (L.) L subsp. annua & Italy & Anti-catarrhal, febrifuge, tonic, vulnerary, against evil eye & Aerial parts & [52] \\
\hline S. arvensis (L.) L. & - & Against evil eye & - & [55] \\
\hline S. balansae Boiss. \& Kotschy & - & Hypotonic diseases, cardiac neuroses & Liquid and alcoholic extracts & [23] \\
\hline \multirow{3}{*}{ S. byzantina K. Koch. } & - & $\begin{array}{l}\text { Anti-inflammatory, antitumor, anticancer, antispasmodic, } \\
\text { sedative and diuretic agent, } \\
\text { and in the treatment of digestive disorders, wounds, } \\
\text { infections, asthma, rheumatic and inflammatory disorders, } \\
\text { dysentery, epilepsy, common cold and neuropathy }\end{array}$ & - & [33] \\
\hline & Iran & Infected wounds, cutting & $\begin{array}{l}\text { Decoction, } \\
\text { Demulcent } \\
\text { (Leaves) }\end{array}$ & {$[34,35]$} \\
\hline & Brazil & Antiinflammatory & Infusion of leaves & [60] \\
\hline S. cretica subsp. anatolica Rech. f. & Turkey & Colds, stomach ailments & Infusion, decoction, internal & [49] \\
\hline $\begin{array}{l}\text { S. cretica L. subsp. mersinaea (Boiss.) } \\
\text { Rech. f. }\end{array}$ & Turkey & Colds, stomach ailments & Infusion, decoction, internal & [49] \\
\hline S. fruticulosa M. Bieb. & Iran & Anti- inflammatory & Aerial parts & [32] \\
\hline S. geobombycis C.Y.Wu & China, Japan and Europe & Tonic & - & [22] \\
\hline \multirow{2}{*}{ S. germanica L. } & Iran & Gastrodynia, for painful menstruation & Infusion of flowers & [34] \\
\hline & - & Skin disorders (Veterinary use) & - & [55] \\
\hline
\end{tabular}


Table 1. Cont

\begin{tabular}{|c|c|c|c|c|}
\hline Species & $\begin{array}{c}\text { Geographical Origin of } \\
\text { the Reported } \\
\text { Traditional Use }\end{array}$ & Traditional Medicinal Use & $\begin{array}{l}\text { Preparation and/or } \\
\text { Administration/ } \\
\text { Parts of the Plant }\end{array}$ & Ref. \\
\hline S. glutinosa L. & - & As antispasmoic and against chicken louse & - & [55] \\
\hline S. iberica subsp. georgica Rech. f. & Turkey & Colds, antipyretic & Decoction, internal & [49] \\
\hline $\begin{array}{l}\text { S. iberica subsp. stenostachya (Boiss.) } \\
\text { Rech. f. }\end{array}$ & Turkey & Colds, antipyretic, stomach ache & Decoction, internal & [49] \\
\hline \multirow{2}{*}{ S. inflata Benth. } & Iran & Infections, asthmatic, rheumatic, inflammatory disorders & $\begin{array}{l}\text { Extracts of aerial parts } \\
\text { (non flowering stems) }\end{array}$ & {$[36,37]$} \\
\hline & Iran & $\begin{array}{l}\text { Common cold, Analgesic, high } \\
\text { blood pressure }\end{array}$ & Decoction of aerial parts & [31] \\
\hline S. iva Griseb. & Greece & Common cold and gastrointestinal disorders & Decoction, infusion & [56] \\
\hline S. kurdica Boiss \& Hohen var. kurdica & Turkey & Cold, stomach-ache & $\begin{array}{l}\text { Decoction of branches/flowers } \\
\text { Drink one glass of the plant on } \\
\text { an empty stomach in } \\
\text { the morning }\end{array}$ & [50] \\
\hline \multirow{6}{*}{ S. lavandulifolia Vahl. } & Iran & Treat pain and inflammation & Boiled extracts of the aerial parts & [12] \\
\hline & Iran & $\begin{array}{l}\text { Sedative, gastrotonic and spasmolytic properties, treatment of } \\
\text { some gastrointestinal disorders, colds and flu }\end{array}$ & $\begin{array}{l}\text { Herbal tea of flowering } \\
\text { aerial parts }\end{array}$ & [13] \\
\hline & Iran & $\begin{array}{c}\text { Headache, renal calculus } \\
\text { common cold, sedative } \\
\text { flavoring agent, abdominal pain }\end{array}$ & $\begin{array}{l}\text { Decoction of aerial parts, } \\
\text { Food additive (aerial parts) }\end{array}$ & {$[31]$} \\
\hline & Turkey & Antipyretic, cough & Decoction, internal & [49] \\
\hline & Iran & Painful and inflammatory disorders & Boiled extracts of aerial parts & [41] \\
\hline & Iran & Anxiolytic influence & Herbal tea & [38-44] \\
\hline
\end{tabular}


Table 1. Cont

\begin{tabular}{|c|c|c|c|c|}
\hline Species & $\begin{array}{l}\text { Geographical Origin of } \\
\text { the Reported } \\
\text { Traditional Use }\end{array}$ & Traditional Medicinal Use & $\begin{array}{l}\text { Preparation and/or } \\
\text { Administration/ } \\
\text { Parts of the Plant }\end{array}$ & Ref. \\
\hline \multirow{4}{*}{ S. mucronata Sieb. } & \multirow{4}{*}{ Greece } & Antirheumatic and antineuralgic remedy & Decoction for massage & \multirow{4}{*}{ [57] } \\
\hline & & For wounds and ulcers & $\begin{array}{l}\text { Washed with the decoction and } \\
\text { covered with a poultice of fresh } \\
\text { leaves for cicatrization }\end{array}$ & \\
\hline & & Antidiarrhoic agent & Infusion of fresh leaves & \\
\hline & & Pugative & Infusion of roots & \\
\hline S. obliqua Waldst. \& Kit. & Turkey & Cold, stomach ailments, fever and cough & Herb, infusion, decoction & [22] \\
\hline \multirow{3}{*}{$\begin{array}{l}\text { S. officinalis (L.) Trevisan (=S. betonica } \\
\text { Benth.; Betonica officinalis L.) }\end{array}$} & $\begin{array}{l}\text { Serbia, Egypt, } \\
\text { Montenegro }\end{array}$ & $\begin{array}{l}\text { Skin disorders, antibacterial purposes, against headache, } \\
\text { nervous tension, anxiety, menopausal problems, as a } \\
\text { tobacco snuff }\end{array}$ & Tea of dried leaves & [22] \\
\hline & Italy & Dye wool yellow & Plant & [51] \\
\hline & Italy & $\begin{array}{c}\text { Wounds, } \\
\text { in the sores of pack animals }\end{array}$ & Oily extract of flowers & [54] \\
\hline \multirow{3}{*}{ S. palustris L. } & - & Disinfectant, anti-spasmodic and for treatment of wounds & - & {$[17,61]$} \\
\hline & Poland & Wounds, additive in food & - & [58] \\
\hline & - & Antiseptic, to relieve gout, to stop haemorrhage & - & [62] \\
\hline $\begin{array}{l}\text { S. parviflora Benth. } \\
\text { (=Phlomidoschema parviflorum } \\
\text { (Benth.) Vved.) }\end{array}$ & - & Cramps, arthralgia, epilepsy, falling sickness, dracunculiasis & - & {$[63,64]$} \\
\hline \multirow{2}{*}{ S. pilifera Benth. } & Iran & $\begin{array}{l}\text { Toothache, edible, tonic, } \\
\text { analgesic, edema, expectorant, tussive }\end{array}$ & Decoction of aerial parts & [31] \\
\hline & Iran & Asthma, rheumatoid arthritis and infections & - & [45] \\
\hline \multirow{3}{*}{ S. pumila Banks \& Sol. } & Anatolia & Antibacterial and healing effects & Tea of the whole part & [21] \\
\hline & Anatolia & Sedative, antispasmodic, diuretic and emmenagogic properties & Tea of the leaves & [21] \\
\hline & - & Bronchitis, asthma, stomach pain and gall and liver disorders & - & [65] \\
\hline
\end{tabular}


Table 1. Cont

\begin{tabular}{|c|c|c|c|c|}
\hline Species & $\begin{array}{l}\text { Geographical Origin of } \\
\text { the Reported } \\
\text { Traditional Use }\end{array}$ & Traditional Medicinal Use & $\begin{array}{l}\text { Preparation and/or } \\
\text { Administration/ } \\
\text { Parts of the Plant }\end{array}$ & Ref. \\
\hline \multirow{4}{*}{ S. recta $\mathrm{L}$. } & Europe & Anxiolytic properties & Herbal tea, Oral administration & [11] \\
\hline & Italy & Headache & Infusion of leaves to wash face & [51] \\
\hline & Italy & Bad influence/spirit & Decoction & [53] \\
\hline & Italy & Depurative & Decoction of the aerial parts & [54] \\
\hline \multirow[b]{2}{*}{ S. recta L. subsp. recta } & \multirow[b]{2}{*}{ Italy } & Tootache and other pain & $\begin{array}{l}\text { Aerial parts applied in } \\
\text { body parts }\end{array}$ & \multirow[b]{2}{*}{ [53] } \\
\hline & & against anxiety, pain and toothache & $\begin{array}{l}\text { Decoction of flowering tops for } \\
\text { bath or to wash face, hands and } \\
\text { wrists for } 3 \text { days }\end{array}$ & \\
\hline \multirow[b]{2}{*}{ S. schtschegleevii Sosn. ex Grossh. } & Iran & Antiinflamatory & Aerial parts & {$[32,34]$} \\
\hline & Iran & $\begin{array}{l}\text { Infectious diseases of the respiratoy tract (for colds and } \\
\text { sinusitis), for asthma, rheumatism and other } \\
\text { inflammatory disorders }\end{array}$ & - & [46] \\
\hline S. sieboldii Miq. (=S. affinis Bunge) & China & Cold and against infections, promoting blood circulation & Dried whole plant & [30] \\
\hline \multirow{3}{*}{ S. sylvatica L. } & - & Disinfectant, anti-spasmodic and for treatment of wounds & - & [17] \\
\hline & Iran & $\begin{array}{l}\text { Diuretic, digestive, emmenagogue, antispasmodic, } \\
\text { anti-inflammatory, sedative, tonic properties and for the } \\
\text { treatment of women with PCOS }\end{array}$ & - & [47] \\
\hline & Turkey & Cardiac disorders & Infusion of aerial parts & [48] \\
\hline S. tibetica Vatke & India & $\begin{array}{l}\text { For fever, cough, phobias and } \\
\text { various mental disorder }\end{array}$ & $\begin{array}{l}\text { Whole plant is boiled and made } \\
\text { into a decoction. Drink one } \\
\text { teacup decoction twice a day to } \\
\text { treat fever for } 5-7 \text { days }\end{array}$ & [66] \\
\hline S. turcomanica Trautv. & Iran & $\begin{array}{l}\text { Foot inflammation, toothache, bronchitis and } \\
\text { common cold }\end{array}$ & $\begin{array}{l}\text { Infusion, Demulcent, Vapor } \\
\text { (Whole plant) }\end{array}$ & [34] \\
\hline
\end{tabular}




\section{Chemical Composition}

Various non-volatile chemical constituents have been reported from different species of genus Stachys, categorizing into important chemical groups including fatty acids, alkaloids (e.g., stachydrine, turiaine), triterpenes, phytosterols, phytoecdysteroids, diterpenes, iridoids, flavonoids, phenylpropanoid glucosides, acetophenones, phenylethanoid glycosides, lignans, phenolic acids, megastigmanes and polysaccharides $[3,20,21,23,67]$. The present survey was focused on all the above groups, excluded fatty acids and alkaloids due to the limited available studies. This section summarizes the phytochemicals from the genus Stachys which are mainly responsible for its pharmacological benefits, presented in Tables 2-15. To be mentioned that large number of phytochemicals were mainly discovered from the aerial parts, leaves and a few were found in stems and roots. 
Table 2. Flavones isolated from Stachys spp

\begin{tabular}{|c|c|c|c|}
\hline Species & Plant Parts & Compound & Ref \\
\hline \multicolumn{4}{|c|}{ Subgenus Stachys } \\
\hline \multicolumn{4}{|c|}{ Section Ambleia } \\
\hline \multirow[t]{4}{*}{ S. aegyptiaca Pers. } & Aerial parts & 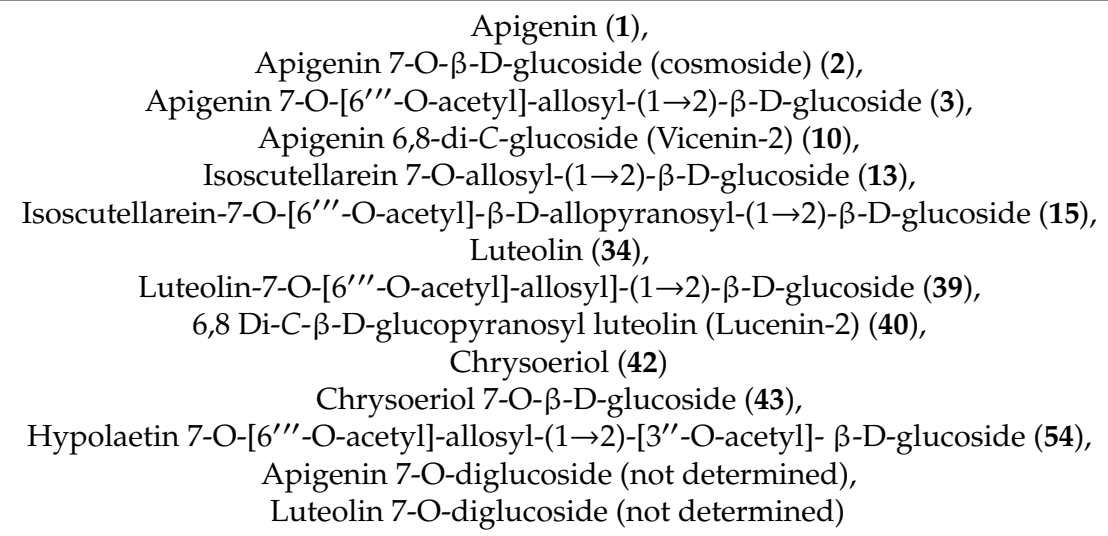 & [68] \\
\hline & Aerial parts & $\begin{array}{l}\text { Apigenin-7-(3"-E- } p \text {-coumaroyl)- } \beta \text {-D-glucoside (4), } \\
\text { Apigenin 7-(6"- }-p \text {-coumaroyl)- } \beta \text {-D-glucoside (6) }\end{array}$ & [69] \\
\hline & Aerial parts & $\begin{array}{c}\text { Isoscutellarein (11), } \\
3^{\prime}, 4^{\prime} \text {-Dimethyl-luteolin-7-O- } \beta \text {-D-glucoside (41) }\end{array}$ & [70] \\
\hline & & Isoscutellarein 8-O-(6" ${ }^{\prime \prime}$ trans- $p$-coumaroyl)- $\beta$-D-glucoside (18) & [71] \\
\hline \multirow[t]{2}{*}{ S. inflata Beth. } & & Scutellarein 7-O- $\beta$-D-mannopyranosyl-( $1 \rightarrow 2)-\beta$-D-glucoside (stachyflaside) (31) & [72] \\
\hline & & Isoscutellarein (11), 4'-Methyl-isoscutellarein (12), Scutellarein (29) & [73] \\
\hline S. schtschegleevii Sosn. ex Grossh. & Stems & $\begin{array}{c}\text { Apigenin 7-O- } \beta \text {-D-glucoside (2), } \\
\text { Apigenin 7-(6"-E- } p \text {-coumaroyl)- } \beta \text {-D-glucopyranoside (6), } \\
\text { 3'-Hydroxy-isoscutellarein-7-O-[6"'"-O-acetyl]- } \beta \text {-D-glucopyranoside (14), } \\
\text { Chrysoeriol 7-(6"-E- } p \text {-coumaroyl)- } \beta \text {-D-glucopyranoside (47) }\end{array}$ & [74] \\
\hline \multicolumn{4}{|c|}{ Section Campanistrum } \\
\hline S. arvensis (L.) L. & Aerial parts \# & 8-Hydroxyflavone-allosylglucosides (not determined) & [75] \\
\hline
\end{tabular}


Table 2. Cont.

\begin{tabular}{|c|c|c|c|}
\hline Species & Plant Parts & Compound & Ref \\
\hline \multicolumn{4}{|c|}{ Subgenus Stachys } \\
\hline \multicolumn{4}{|c|}{ Section Campanistrum } \\
\hline \multirow[t]{2}{*}{ S. ocymastrum (L.) Briq. (= S. hirta L.) } & Aerial parts \# & 8-Hydroxyflavone-allosylglucosides (not determined) & [75] \\
\hline & Aerial parts & $\begin{array}{c}\text { Apigenin (1), } \\
\text { Apigenin 7-(6"-E-p-coumaroyl)- } \beta \text {-D-glucopyranoside (6), } \\
\text { Isoscutellarein 7-O-allosyl-(1 } \rightarrow 2) \text { - glucopyranoside (13), } \\
\text { Luteolin (34) }\end{array}$ & [76] \\
\hline \multicolumn{4}{|c|}{ Section Candida } \\
\hline \multirow[t]{2}{*}{ S. candida Bory \& Chaubard } & Aerial parts & Chrysoeriol (42), Chrysoeriol 7-(3"'-E- $p$-coumaroyl)- $\beta$-D-glucopyranoside (46) & [77] \\
\hline & Aerial parts & 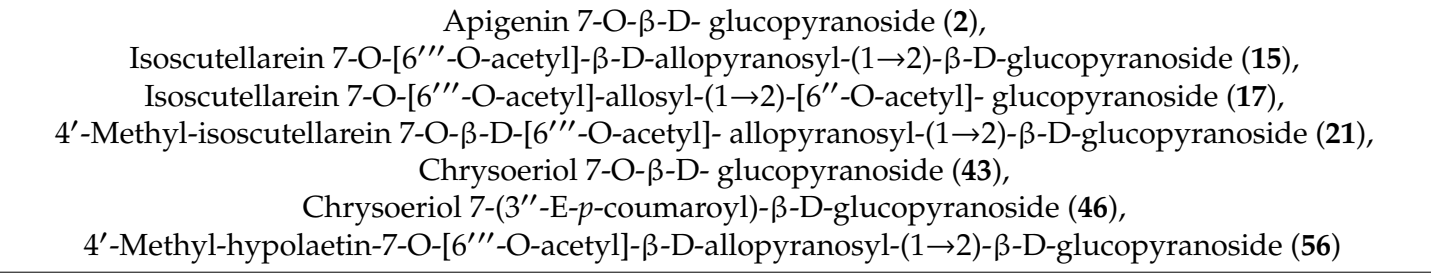 & [78] \\
\hline S. chrysantha Boiss. and Heldr. & Aerial parts & 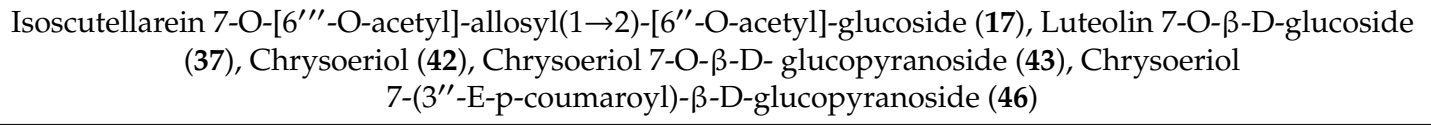 & [77] \\
\hline S. iva Griseb. & $\begin{array}{l}\text { Flowering } \\
\text { aerial parts }\end{array}$ & 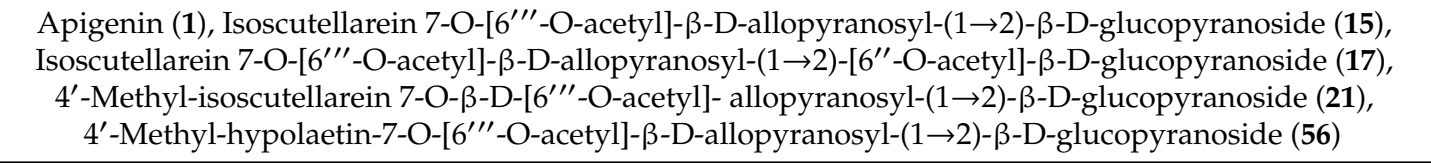 & [56] \\
\hline
\end{tabular}


Table 2. Cont

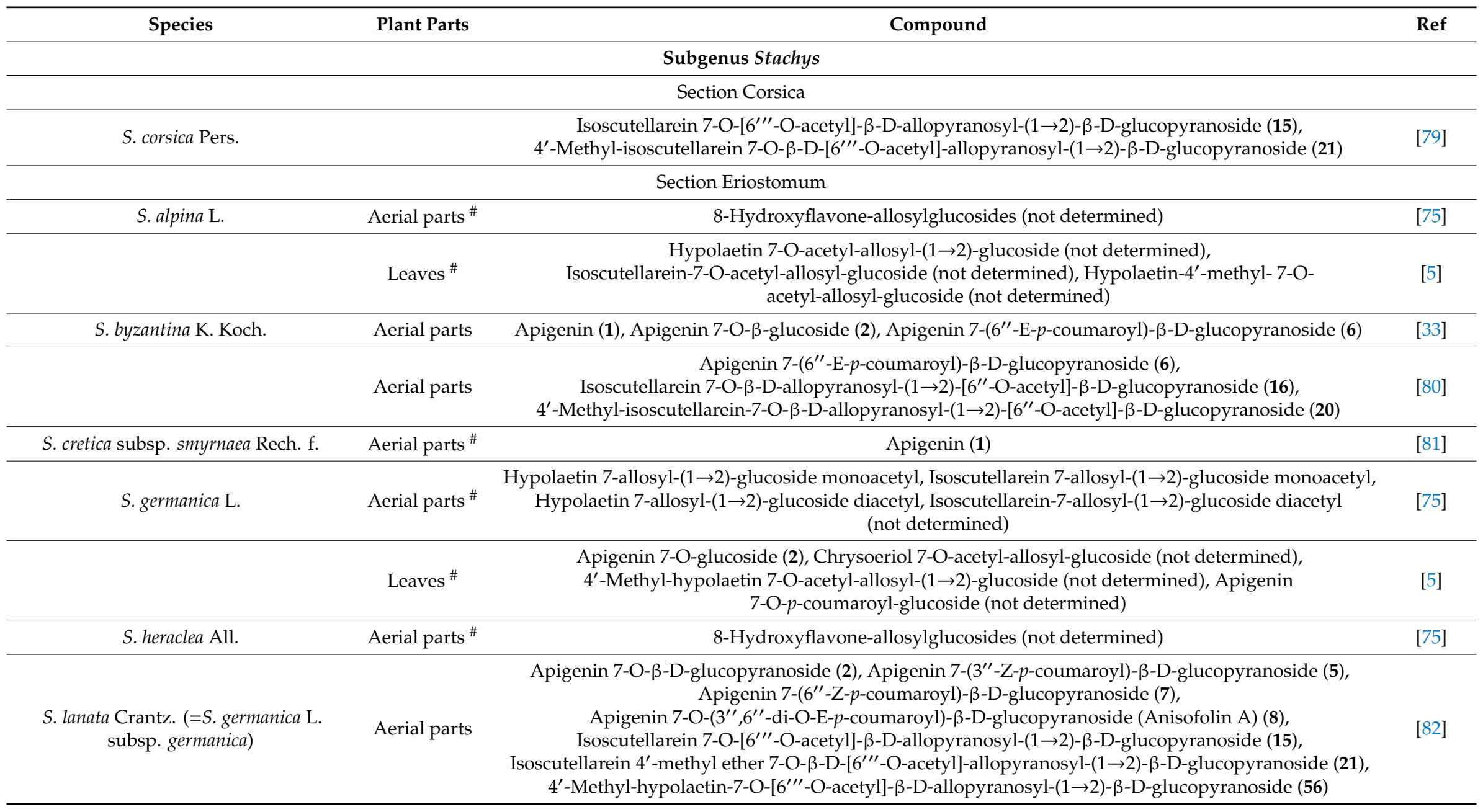


Table 2. Cont

\begin{tabular}{|c|c|c|c|}
\hline Species & Plant Parts & Compound & Ref \\
\hline \multicolumn{4}{|c|}{ Subgenus Stachys } \\
\hline \multicolumn{4}{|c|}{ Section Eriostomum } \\
\hline S. spectabilis Choisy ex DC. & Epigeal parts & $\begin{array}{c}\text { Isostachyflaside (25), } \\
\text { Spectabiflaside (28), Scutellarein 7-O- } \beta \text {-D-mannopyranosyl-(1 } \rightarrow 2)-\beta-D-\text { glucopyranoside (stachyflaside) }\end{array}$ & [83] \\
\hline S. thirkei K. Koch. & Whole plant $\#$ & Apigenin (1) & {$[84]$} \\
\hline S. tmolea Boiss. & Aerial parts \# & Apigenin (1), Apigenin-7-O-glucoside (2) & {$[85]$} \\
\hline $\begin{array}{l}\text { S. tymphaea Hausskn. (=S. germanica } \\
\text { subsp. tymphaea (Hausskn.) } \\
\text { R. Bhattacharjee) }\end{array}$ & $\begin{array}{l}\text { Flowering } \\
\text { aerial parts }\end{array}$ & 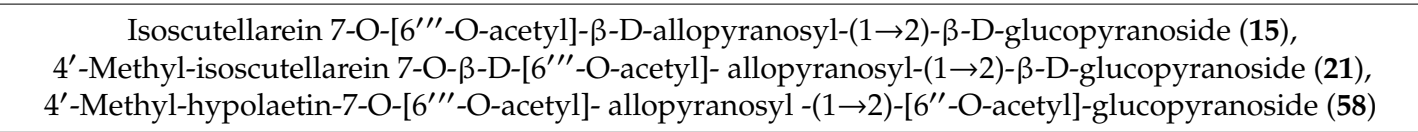 & {$[86]$} \\
\hline \multicolumn{4}{|c|}{ Section Fragilicaulis } \\
\hline S. subnuda Montbret \& Aucher ex Benth & Aerial parts & 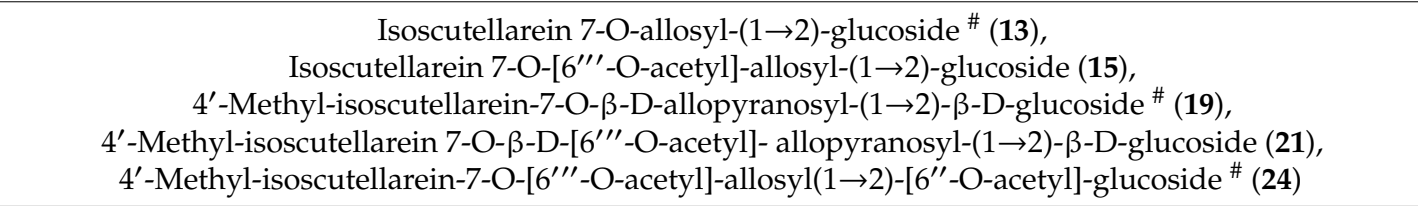 & [87] \\
\hline \multicolumn{4}{|c|}{ Section Olisia } \\
\hline \multirow{5}{*}{ S. atherocalyx C. Koch } & & Stachyflaside (31) & {$[72]$} \\
\hline & & Diacetylstachyflaside (not determined), Diacetylspectabiflaside (not determined), Spectabiflaside (28) & [88] \\
\hline & & $\begin{array}{c}\text { 5,8, } 4^{\prime} \text {-Trihydroxy-3'-methoxy-7-O-( } \beta \text {-D-glucopyranosyl-2" }- \text {-O- } \beta \text {-D-mannopyranosyl)-flavone } \\
\text { (Spectabiflaside) (28), Acetyl-sectabiflaside (not determined), }\end{array}$ & [89] \\
\hline & & $\begin{array}{l}\text { Acetyl-isostachyflaside (26), Di-acetyl-isostachyflaside (27), } \\
\text { Spectabiflaside (28) }\end{array}$ & {$[90]$} \\
\hline & Leaves $\#$ & 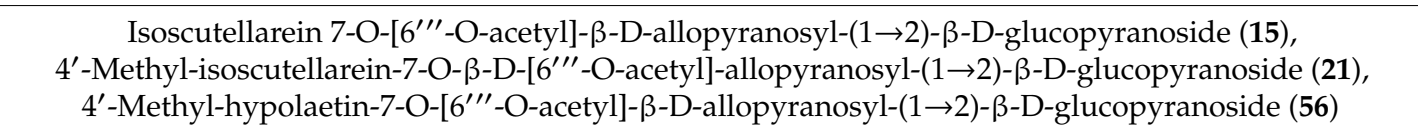 & [91] \\
\hline S. angustifolia M. Bieb. & & $\begin{array}{l}\text { Isoscutellarein 7-O-[6"' -O-acetyl]- } \beta \text {-D-allopyranosyl-( } 1 \rightarrow 2)-\beta \text {-D-glucopyranoside (15), } \\
4^{\prime} \text {-Methyl-isoscutellarein 7-O- } \beta \text {-D-[6" } 6^{\prime \prime}-\mathrm{O} \text {-acetyl]-allopyranosyl- }(1 \rightarrow 2)-\beta \text {-D-glucopyranoside (21) }\end{array}$ & [92] \\
\hline
\end{tabular}


Table 2. Cont

\begin{tabular}{|c|c|c|c|}
\hline Species & Plant Parts & Compound & Ref \\
\hline \multicolumn{4}{|c|}{ Subgenus Stachys } \\
\hline \multicolumn{4}{|c|}{ Section Olisia } \\
\hline \multirow{5}{*}{ S. annua (L.) L. } & Epigeal parts & 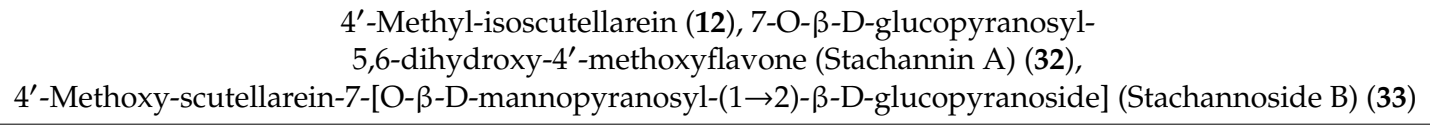 & [93] \\
\hline & Leaves \# & 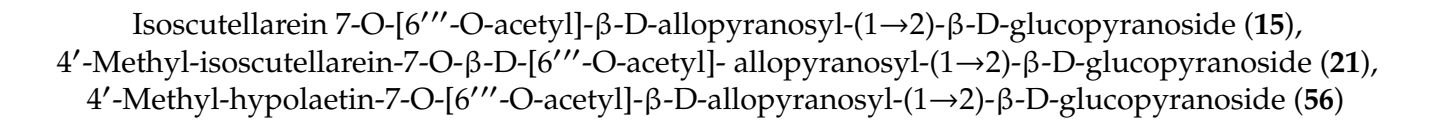 & [92] \\
\hline & Aerial parts & 4'-Methyl-isoscutellarein 7-O- $\beta$-D-[6"' -O-acetyl]-allopyranosyl-( $1 \rightarrow 2)$ - $\beta$-D-glucopyranoside (21) & [94] \\
\hline & Aerial parts & 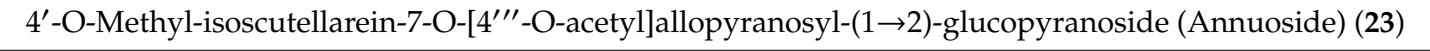 & [95] \\
\hline & $\begin{array}{l}\text { Subterranean } \\
\text { organs }\end{array}$ & 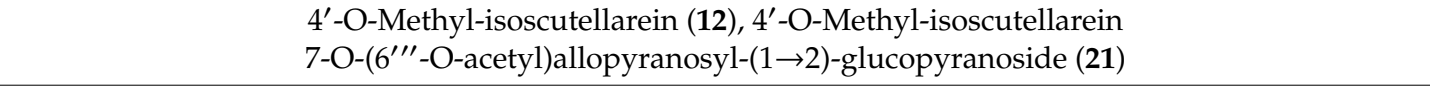 & [95] \\
\hline S.annua (L.) L. subsp. апnиa & $\begin{array}{l}\text { Flowering } \\
\text { aerial parts }\end{array}$ & 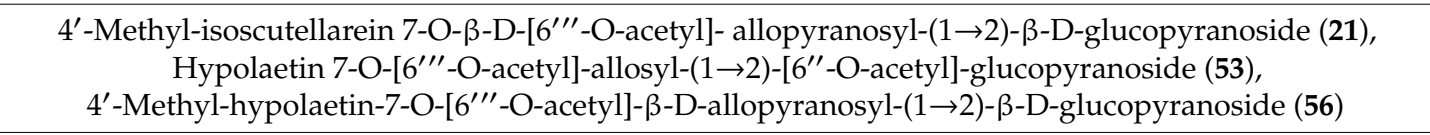 & [52] \\
\hline S. beckeana Dörfler \& Hayek & Leaves \# & 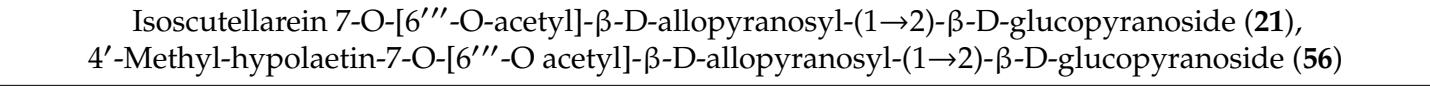 & [92] \\
\hline S. bombycina Boiss. & Aerial parts & Apigenin 7-(6"-E-p-coumaroyl)- $\beta$-D-glucopyranoside (6), Stachyspinoside (44) & [96] \\
\hline S. parolinii Vis. & Leaves \# & 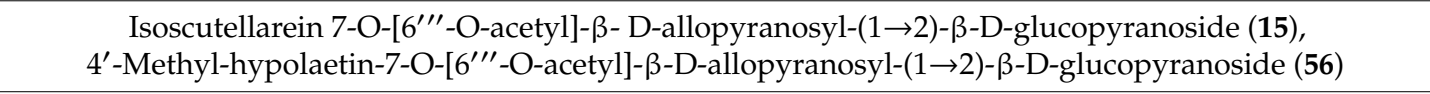 & [92] \\
\hline S. leucoglossa Griseb. & Leaves \# & 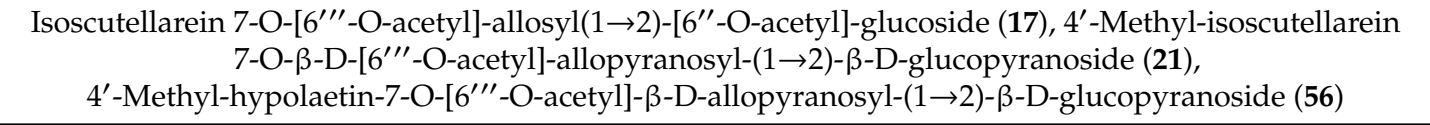 & [92] \\
\hline
\end{tabular}


Table 2. Cont

\begin{tabular}{|c|c|c|c|}
\hline Species & Plant Parts & Compound & Ref \\
\hline \multicolumn{4}{|c|}{ Subgenus Stachys } \\
\hline \multicolumn{4}{|c|}{ Section Olisia } \\
\hline $\begin{array}{l}\text { S. neglecta Klok. ex Kossko } \\
\text { (=S. annua (L.) L.) }\end{array}$ & & $\begin{array}{l}\text { Apigenin (1), } \\
\text { Apigenin 7-O- } \beta \text {-D-glucoside (2), Luteolin (34), Luteolin 7-O- } \beta \text {-D-glucoside (37) }\end{array}$ & [97] \\
\hline \multirow[b]{2}{*}{ S. recta $\mathrm{L}$. } & Leaves & 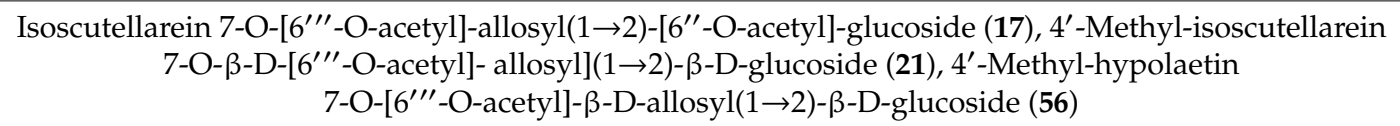 & {$[91,92]$} \\
\hline & Aerial parts & 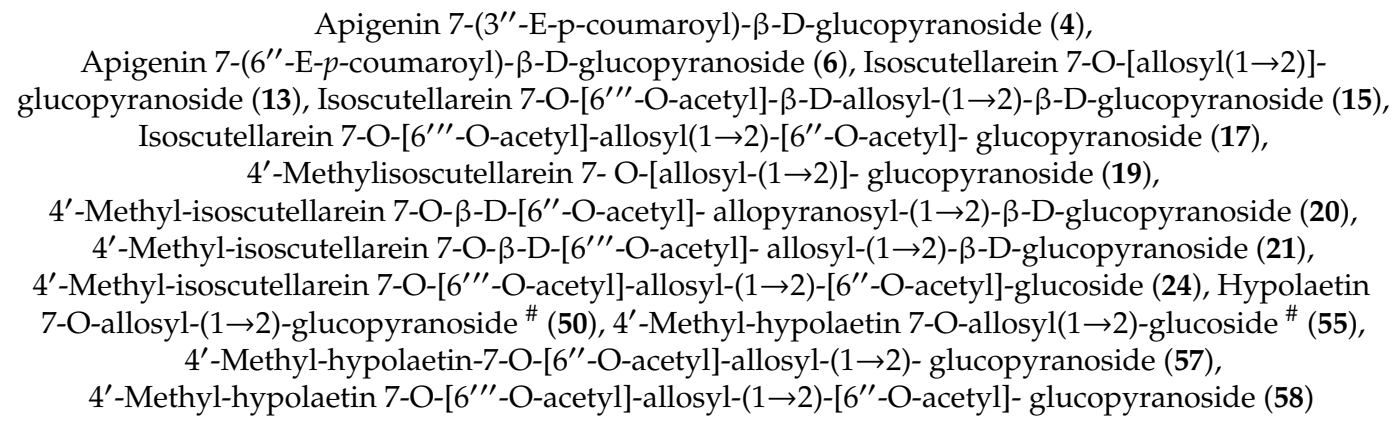 & [14] \\
\hline $\begin{array}{l}\text { S. labiosa Bertol. (=S. recta subsp. } \\
\text { labiosa (Bertol.) Briq.) }\end{array}$ & Leaves & 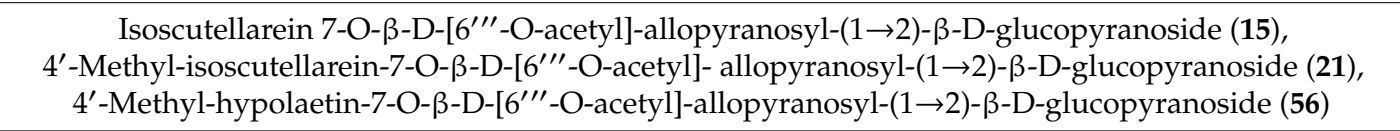 & [92] \\
\hline $\begin{array}{l}\text { S. subcrenata Vis.(=S. recta L. subsp. } \\
\text { subcrenata (Vis.) Briq.) }\end{array}$ & Leaves & 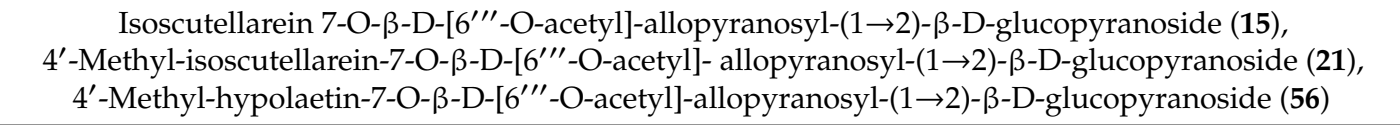 & [92] \\
\hline $\begin{array}{l}\text { S. baldaccii (Maly) Hand.-Mazz. } \\
\text { (=S. recta L. subsp. baldaccii } \\
\text { (K. Maly) Hayek) }\end{array}$ & Leaves \# & 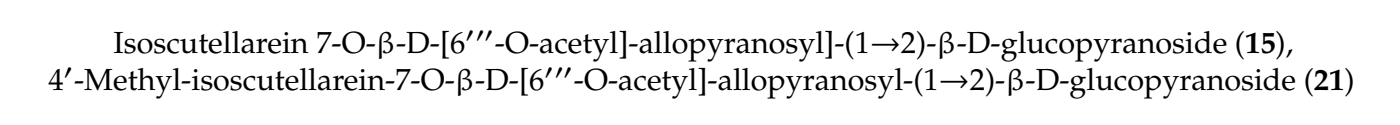 & [92] \\
\hline
\end{tabular}


Table 2. Cont

\begin{tabular}{|c|c|c|c|}
\hline Species & Plant Parts & Compound & Ref \\
\hline \multicolumn{4}{|c|}{ Subgenus Stachys } \\
\hline \multicolumn{4}{|c|}{ Section Olisia } \\
\hline \multirow{2}{*}{ S. spinosa L. } & Aerial parts & Chrysoeriol 7-O-[6"' -O-acetyl-allosyl]-(1 $\rightarrow 2)$-glucoside (Stachyspinoside) (44) & [98] \\
\hline & Aerial parts & Chrysoeriol 7-O-[6"'-O-acetyl-allosyl]-(1 $\rightarrow 2)$-glucoside (Isostachyspinoside) (45) & [99] \\
\hline \multirow{2}{*}{ S. tetragona Boiss. \& Hayek } & Leaves \# & $\begin{array}{l}\text { Isoscutellarein 7-O-[6"' }-\mathrm{O} \text {-acetyl]- } \beta \text {-D-allopyranosyl-( } 1 \rightarrow 2)-\beta \text {-D-glucopyranoside (15), } \\
4^{\prime} \text {-Methyl-isoscutellarein 7-O- } \beta \text {-D-[6'" }-\mathrm{O} \text {-acetyl]-allopyranosyl- }(1 \rightarrow 2)-\beta \text {-D-glucopyranoside (21) }\end{array}$ & [92] \\
\hline & Aerial parts & 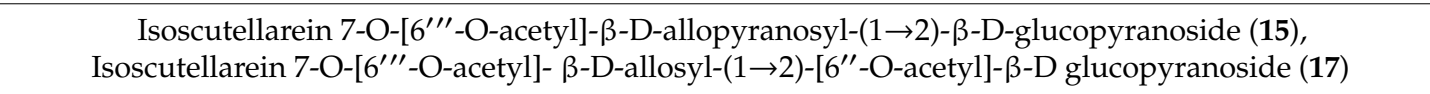 & {$[100]$} \\
\hline \multicolumn{4}{|c|}{ Section Swainsoniana } \\
\hline \multirow[t]{2}{*}{ S. anisochila Vis. \& Pancic } & Leaves & 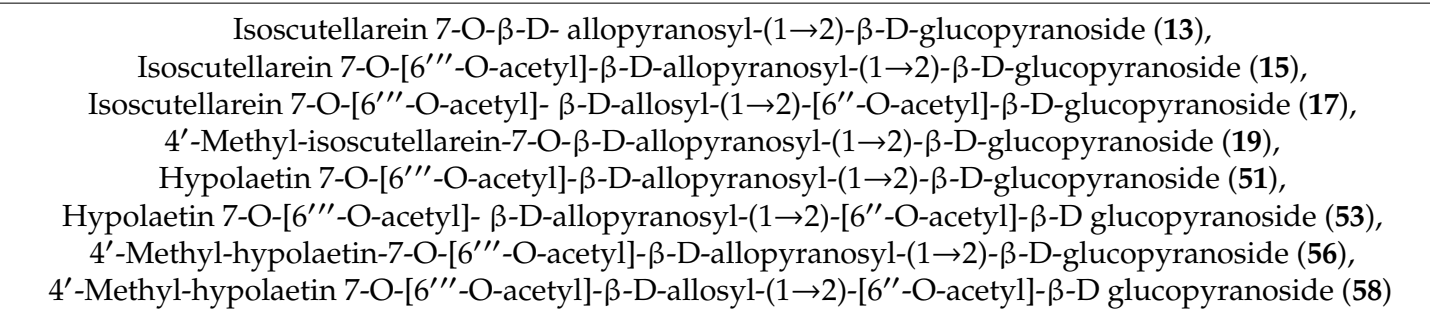 & {$[101]$} \\
\hline & Leaves & Apigenin 7-O-( $p$-coumaroyl)- $\beta$-D-glucopyranoside (not determined) & [5] \\
\hline $\begin{array}{l}\text { S. decumbens Pers. } \\
\text { (=S. mollissima Willd.) }\end{array}$ & Aerial parts \# & 8-Hydroxyflavone-allosylglucosides (not determined) & [75] \\
\hline S. menthifolia Vis. (=S. grandiflora Host.) & Leaves \# & 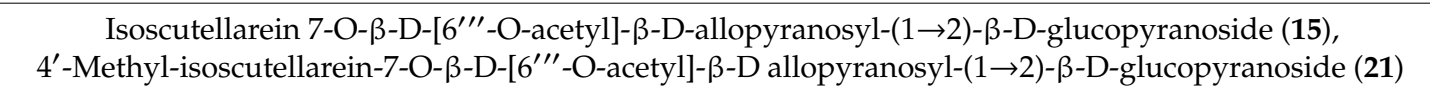 & [92] \\
\hline S. swainsonii Benth. subsp. swainsonii & Aerial parts & $\begin{array}{c}\text { Apigenin (1), Apigenin 7-O- } \beta \text {-D-glucopyranoside (2), Apigenin 7-O- } \beta \text {-D-glucoside (2), Luteolin } \\
\text { 7-O- } \beta \text {-D-glucopyranoside (37), Chrysoeriol (42), Chrysoeriol 7-O- } \beta \text {-D-glucopyranoside (43), } \\
\text { Stachyspinoside (44) }\end{array}$ & {$[102]$} \\
\hline $\begin{array}{l}\text { S. swainsonii subsp. argolica (Boiss.) } \\
\text { Phitos and Damboldt }\end{array}$ & Aerial parts & $\begin{array}{l}\text { Apigenin (1), Luteolin 7-O- } \beta \text {-D-glucopyranoside (37), Chrysoeriol (42), } \\
\text { Chrysoeriol-7-O- } \beta \text {-D-glucopyranoside (43), Chrysoeriol 7-(3"'-E- } p \text {-coumaroyl)- } \beta \text {-D-glucopyranoside (46) }\end{array}$ & {$[102]$} \\
\hline $\begin{array}{l}\text { S. swainsonii subsp. melangavica } \\
\text { D. Persson }\end{array}$ & Aerial parts & $\begin{array}{l}\text { Apigenin (1), Apigenin 7-O- } \beta \text {-D- glucopyranoside (2), Luteolin 7-O- } \beta \text {-D-glucopyranoside (37), } \\
\text { Chrysoeriol-7-O- } \beta \text {-D-glucopyranoside (43), Stachyspinoside (44) }\end{array}$ & {$[102]$} \\
\hline
\end{tabular}


Table 2. Cont

\begin{tabular}{|c|c|c|c|}
\hline Species & Plant Parts & Compound & Ref \\
\hline \multicolumn{4}{|c|}{ Subgenus Stachys } \\
\hline \multicolumn{4}{|c|}{ Section Swainsoniana } \\
\hline $\begin{array}{l}\text { S. swainsonii subsp. scyronica (Boiss.) } \\
\text { Phitos and Damboldt }\end{array}$ & Aerial parts & $\begin{array}{l}\text { Apigenin (1), Apigenin 7-O- } \beta \text {-D- glucopyranoside (2), Luteolin 7-O- } \beta \text {-D-glucopyranoside (37), } \\
\text { Chrysoeriol-7-O- } \beta \text {-D-glucopyranoside (43), Stachyspinoside (44) }\end{array}$ & [102] \\
\hline S. ionica Halácsy & Aerial parts & 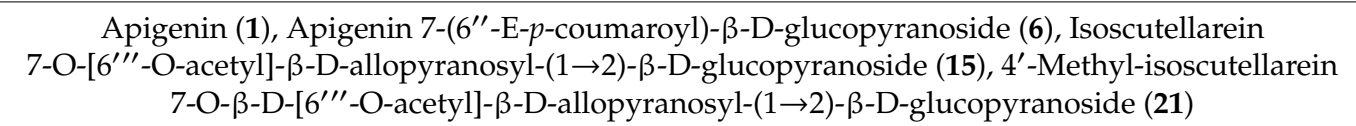 & {$[20]$} \\
\hline \multicolumn{4}{|c|}{ Section Stachys } \\
\hline S. sieboldii Miq. (=S. affinis Bunge) & Aerial parts & 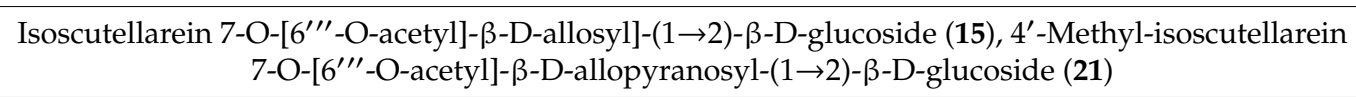 & {$[20]$} \\
\hline S. mialhesii Noé & Aerial parts & 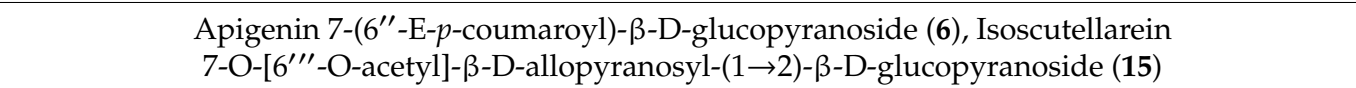 & [103] \\
\hline \multirow[t]{2}{*}{ S. palustris L. } & & $\begin{array}{l}\text { 5-(glycuroglucosyl)-7-methoxybaicalein (Palustrin) (63), 5-(glucuronosyl)-7-methoxybaicalein } \\
\text { (Palustrinoside) (64) }\end{array}$ & [104] \\
\hline & Leaves \# & Vicenin-2 (10), Apigenin 7-O- $p$-coumaroyl- $\beta$-D-glucopyranoside (not determined) & [5] \\
\hline \multirow[b]{2}{*}{ S. sylvatica $\mathrm{L}$. } & Aerial parts $\#$ & 8-Hydroxyflavone-allosyl-glucosides (not determined) & [75] \\
\hline & Leaves \# & $\begin{array}{l}\text { Chrysoeriol 7-O-acetylallosylglucoside (not determined), Apigenin } \\
\text { 7-O- } p \text {-coumaroyl- } \beta \text {-D-glucopyranoside (not determined) }\end{array}$ & [5] \\
\hline S. plumosa Griseb. & Leaves \# & $\begin{array}{l}\text { Apigenin-7-O- } \beta \text {-D-glucoside (2), Luteolin 7-O- } \beta \text {-D-glucoside (37), Chrysoeriol } \\
\text { 7-O-acetyl-allosyl-glucoside (not determined), Isoscutellarein 7-O-acetyl-allosyl-glucoside (not } \\
\text { determined), Apigenin 7-O- } p \text {-coumaroyl- } \beta \text {-D-glucopyranoside (not determined) }\end{array}$ & [5] \\
\hline \multicolumn{4}{|c|}{ Section Zietenia } \\
\hline S. lavandulifolia Vahl. & Aerial parts & Apigenin (1), Hydroxygenkwanin (Luteolin 7-Methyl ether) (35), Chrysoeriol (42) & [13] \\
\hline S. tibetica Vatke & Roots & Apigenin $7-\mathrm{O}-\beta$-D-glucoside (2) & [66] \\
\hline
\end{tabular}


Table 2. Cont

\begin{tabular}{|c|c|c|c|}
\hline Species & Plant Parts & Compound & Ref \\
\hline \multicolumn{4}{|c|}{ Subgenus Betonica } \\
\hline \multicolumn{4}{|c|}{ Section Betonica } \\
\hline & Aerial parts & $p$-coumaroyl-glucosides (not determined) $\#$ & {$[75]$} \\
\hline S. alopecuros (L.) Benth. & Leaves \# & $\begin{array}{c}\text { Isoscutellarein 7-O-glucoside (11a), } \\
\text { Luteolin 7-O-glucuronide (36), } \\
\text { Luteolin 7-O-glucoside (37), Chrysoeriol 7-O-glucoside (43), } \\
\text { Hypolaetin 7-O-glucoside (49), } \\
\text { Hypolaetin 7-O-glucuronide (49a), } \\
\text { Selgin 7-O-glucoside (59), } \\
\text { Tricin 7-O-glucuronide (60), } \\
\text { Tricin 7-O-glucoside (61), } \\
\text { Apigenin 7-O-p-coumaroyl glucopyranoside (not determined) }\end{array}$ & [5] \\
\hline $\begin{array}{l}\text { S. foliosa Regel. (=S. betoniciflora Rupr.; } \\
\text { Betonica foliosa Rupr.) }\end{array}$ & & Four flavonoids (not determined) & {$[105$} \\
\hline $\begin{array}{l}\text { S. monieri (Gouan) P.W. Ball. } \\
\text { (=S. officinalis (L.) Trevis } \\
\text { subsp. officinalis) }\end{array}$ & Aerial parts & $p$-coumaroyl-glucosides (not determined) $\#$ & [75] \\
\hline \multirow{3}{*}{$\begin{array}{l}\text { S. officinalis (L.) Trevis } \\
\text { (=Betonica officinalis L.) }\end{array}$} & & $\begin{array}{c}\text { Apigenin (1), } \\
\text { 5, 6, 4' -trihydroxyflavone-7-O- } \beta \text {-D-glucoside (30) }\end{array}$ & [20] \\
\hline & Leaves \# & $\begin{array}{c}\text { Apigenin 8-C-glucoside } \\
\text { (Vitexin) (9), Luteolin 7-O-glucuronide (36), } \\
\text { Luteolin 6-C-glucoside (isoorientin) (38), } \\
\text { Tricin 7-O-glucuronide (60), } \\
\text { Tricin 7-O-glucoside (61), } \\
\text { Tricetin 3', } 4^{\prime}, 5^{\prime} \text {-trimethyl-7-O-glucoside (62), } \\
\text { Apigenin 7-O-p-coumaroyl glucopyranoside (not determined) }\end{array}$ & [5] \\
\hline & Aerial parts & p-coumaroyl-glucosides (not determined) $\#$ & [75] \\
\hline
\end{tabular}


Table 2. Cont

\begin{tabular}{|c|c|c|c|}
\hline Species & Plant Parts & Compound & Ref \\
\hline \multicolumn{4}{|c|}{ Subgenus Betonica } \\
\hline \multicolumn{4}{|c|}{ Section Macrostachya } \\
\hline $\begin{array}{c}\text { S. scardica Griseb. } \\
\text { (=Betonica scardica Griseb.) }\end{array}$ & Leaves \# & $\begin{array}{c}\text { Apigenin 8-C-glucoside (9), Luteolin 7-O-glucoside (37), } \\
\text { Luteolin 6-C-glucoside (38), } \\
\text { Hypolaetin 7-O-glucoside (49), } \\
\text { Selgin 7-O-glucoside (59), } \\
\text { Tricin 7-O-glucuronide (60), } \\
\text { Tricin 7-O-glucoside (61), } \\
\text { Tricetin 3', } 4^{\prime}, 5^{\prime} \text {-trimethyl-7-O-glucoside (isolation) (62), } \\
\text { Apigenin 7-O- } p \text {-coumaroyl glucopyranoside (not determined) }\end{array}$ & [5] \\
\hline
\end{tabular}

\# identified compounds by means of HPLC, LC-MS, etc. 
Table 3. Poly-methylated flavonoids from Stachys spp.

\begin{tabular}{|c|c|c|c|}
\hline Species & Plant Parts & Compound & Ref \\
\hline \multicolumn{4}{|c|}{ Subgenus Stachys } \\
\hline \multicolumn{4}{|c|}{ Section Ambleia } \\
\hline \multirow{3}{*}{ S. aegyptiaca Pers. } & Aerial parts & 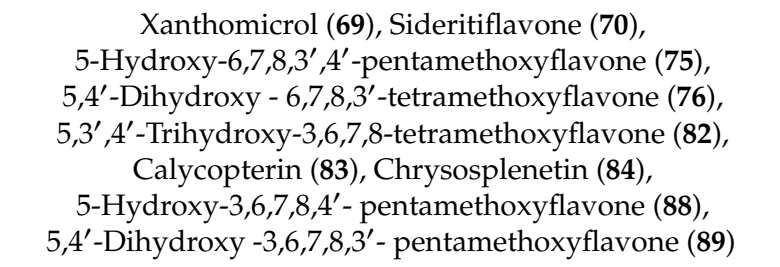 & [68] \\
\hline & Aerial parts & $\begin{array}{l}\text { 5,7,3'-Trihydroxy-6, } 4^{\prime} \text {-dimethoxyflavone }(67) \text {, } \\
5,7,3^{\prime} \text {-Trihydroxy-6,8,4'-trimethoxyflavone (68) }\end{array}$ & [70] \\
\hline & Aerial parts & $\begin{array}{c}\text { Xanthomicrol (69), Eupatilin-7-methyl ether (73), } \\
\text { Calycopterin (83), 5-Hydroxy-3,6,7,4'-tetramethoxy } \\
\text { flavone (85), 5,8-Dihydroxy-3,6,7,4'-tetramethoxy flavone } \\
\text { (86), 5-Hydroxy-auranetin }(\mathbf{8 8}), 4^{\prime} \text {-Hydroxy-3,5,7,3'- } \\
\text { tetramethoxy flavone (90) }\end{array}$ & [106] \\
\hline $\begin{array}{l}\text { S. schtschegleevii Sosn. } \\
\text { ex Grossh. }\end{array}$ & Stems & Cirsimaritin (66), Xanthomicrol (69) & [74] \\
\hline \multicolumn{4}{|c|}{ Section Aucheriana } \\
\hline S. glutinosa L. & & $\begin{array}{l}\text { Xanthomicrol (69), Sideritiflavone (70), } \\
\text { 8-Methoxycirsilineol (71), Eupatilin (72a) }\end{array}$ & [107] \\
\hline \multicolumn{4}{|c|}{ Section Candida } \\
\hline $\begin{array}{l}\text { S. candida Bory \& } \\
\text { Chaubard }\end{array}$ & Aerial parts & Xanthomicrol (69), Calycopterin (83) & {$[77,78]$} \\
\hline $\begin{array}{l}\text { S. chrysantha Boiss. and } \\
\text { Heldr. }\end{array}$ & Aerial parts & Xanthomicrol (69), Calycopterin (83) & [77] \\
\hline \multicolumn{4}{|c|}{ Section Swainsoniana } \\
\hline $\begin{array}{l}\text { S. swainsonii Benth. } \\
\text { subsp. swainsonii }\end{array}$ & Aerial parts & Eupatorin (72), Penduletin (81), 5-Hydroxyauranetin (88) & [102] \\
\hline $\begin{array}{l}\text { S. swainsonii subsp. } \\
\text { argolica (Boiss.) Phitos } \\
\text { and Damboldt }\end{array}$ & Aerial parts & $\begin{array}{l}\text { Xanthomicrol (69), Eupatorin (72), } \\
\text { Salvigenin (74) }\end{array}$ & [102] \\
\hline $\begin{array}{l}\text { S. swainsonii subsp. } \\
\text { melangavica D. Persson }\end{array}$ & Aerial parts & Eupatorin (72), 5-Hydroxyauranetin (88) & [102] \\
\hline $\begin{array}{l}\text { S. swainsonii subsp. } \\
\text { scyronica (Boiss.) Phitos } \\
\text { and Damboldt }\end{array}$ & Aerial parts & Eupatorin (72), Penduletin (81), 5-Hydroxyauranetin (88) & [102] \\
\hline S. ionica Halácsy & Aerial parts & $\begin{array}{l}\text { Xanthomicrol (69), Salvigenin (74), Chrysosplenetin (84), } \\
\text { 5-Hydroxy-3,6,7,4'-tetramethoxyflavone (85), Casticin (87) }\end{array}$ & [20] \\
\hline S. lavandulifolia Vahl. & Aerial parts & $\begin{array}{l}\text { Velutin (Luteolin 7,3'-dimethyl ether) (65), Viscosine } \\
\text { (5,7,4'-trihydroxy-3,6-dimethoxyflavone (78), } \\
\text { Kumatakenin (Kaempferol 3,7-dimethyl ether) (79), } \\
\text { Pachypodol (Quercetin 3,7,3'-trimethyl ether) (80), } \\
\text { Penduletin (81), Chrysosplenetin (84), }\end{array}$ & [13] \\
\hline \multicolumn{4}{|c|}{ Subgenus Betonica } \\
\hline \multicolumn{4}{|c|}{ Section Betonica } \\
\hline $\begin{array}{l}\text { S. officinalis (L.) Trevis = } \\
\text { (Betonica officinalis L.) }\end{array}$ & & 5,4'-Dyhydroxy-7, $3^{\prime}, 5^{\prime}$-trimethoxyflavone (77) & [20] \\
\hline
\end{tabular}


Table 4. Flavonols from Stachys spp.

\begin{tabular}{|c|c|c|c|}
\hline Species & Plant Parts & Compound & Ref \\
\hline \multicolumn{4}{|c|}{ Subgenus Stachys } \\
\hline \multicolumn{4}{|c|}{ Section Eriostomum } \\
\hline S. cretica subsp. smyrnaea Rech. f. & Aerial parts \# & Kaempferol (91) & [81] \\
\hline \multicolumn{4}{|c|}{ Section Olisia } \\
\hline S. tetragona Boiss. \& Hayek & Aerial parts & Kaempferol (91) & [100] \\
\hline \multicolumn{4}{|c|}{ Section Swainsoniana } \\
\hline S. swainsonii Benth. subsp. swainsonii & Aerial parts & Isorhamnetin (92) & [99] \\
\hline $\begin{array}{l}\text { S. swainsonii subsp. argolica (Boiss.) } \\
\text { Phitos and Damboldt }\end{array}$ & Aerial parts & Isorhamnetin (92) & [99] \\
\hline \multicolumn{4}{|c|}{ Section Stachys } \\
\hline S. palustris L. & Leaves \# & $\begin{array}{l}\text { Quercetin-3-O-rutinoside (93), } \\
\text { Isorhamnetin-3-O-rutinoside (94) }\end{array}$ & {$[5]$} \\
\hline
\end{tabular}

Table 5. Flavanones from Stachys spp.

\begin{tabular}{|c|c|c|c|}
\hline Species & Plant Parts & Compound & Ref \\
\hline \multicolumn{4}{|c|}{ Subgenus Stachys } \\
\hline \multicolumn{4}{|c|}{ Section Ambleia } \\
\hline S. aegyptiaca Pers. & Aerial parts & Naringenin (96) & [69] \\
\hline \multicolumn{4}{|c|}{ Section Eriostomum } \\
\hline S. cretica subsp. smyrnaea Rech. f. & Aerial parts \# & Hesperidin (97) & {$[81]$} \\
\hline \multicolumn{4}{|c|}{ Section Swainsoniana } \\
\hline S. swainsonii Benth. subsp. swainsonii & Aerial parts & Eriodictyol (95) & [102] \\
\hline S. swainsonii subsp. argolica (Boiss.) Phitos and Damboldt & Aerial parts & Eriodictyol (95) & [102] \\
\hline S. swainsonii subsp. melangavica D. Persson & Aerial parts & Eriodictyol (95) & [102] \\
\hline S. swainsonii subsp. scyronica (Boiss.) Phitos and Damboldt & Aerial parts & Eriodictyol (95) & [102] \\
\hline
\end{tabular}

Table 6. Biflavonoid from Stachys spp.

\begin{tabular}{|c|c|c|c|}
\hline Species & Plant Parts & Compound & Ref \\
\hline \multicolumn{4}{|c|}{ Subgenus Stachys } \\
\hline \multicolumn{4}{|c|}{ Section Ambleia } \\
\hline S. aegyptiaca Pers. & Aerial Parts & $\begin{array}{c}\text { Diapigenin-7-O-(6" } 6^{\prime \prime} \text {-trans, } 6^{\prime \prime} \text {-cis- } p \\
p^{\prime} \text {-dihydroxy- } \mu \text {-truxinyl)glucoside (stachysetin) (98) }\end{array}$ & [69] \\
\hline \multicolumn{4}{|c|}{ Section Eriostomum } \\
\hline $\begin{array}{c}\text { S. lanata Crantz. } \\
(=\text { S. germanica L. subsp. } \\
\text { germanica) }\end{array}$ & Aerial parts & Stachysetin (98) & [82] \\
\hline \multicolumn{4}{|c|}{ Section Candida } \\
\hline S. iva Griseb. & $\begin{array}{l}\text { Flowering } \\
\text { aerial parts }\end{array}$ & Stachysetin (98) & [56] \\
\hline
\end{tabular}


Table 7. Phenolic derivatives from Stachys spp.

\begin{tabular}{|c|c|c|c|}
\hline Species & Plant Parts & Compound & Ref \\
\hline \multicolumn{4}{|c|}{ Subgenus Stachys } \\
\hline \multicolumn{4}{|c|}{ Section Candida } \\
\hline S. candida Bory \& Chaubard & Aerial parts & Chlorogenic acid (103) & [78] \\
\hline S. iva Griseb & Flowering aerial parts & Chlorogenic acid (103) & [56] \\
\hline \multicolumn{4}{|c|}{ Section Eriostomum } \\
\hline S. cretica subsp. smyrnaea Rech. f. & Aerial parts \# & Chlorogenic acid (103) & [81] \\
\hline S. cretica subsp. vacillans Rech. f. & Aerial parts \# & $\begin{array}{c}\text { Vanillic acid (100), } \\
\text { Syringic acid (101), } \\
\text { Chlorogenic acid (103) }\end{array}$ & {$[105]$} \\
\hline S. cretica subsp. mersinaea (Boiss.) Rech. f. & Aerial parts \# & Chlorogenic acid (103) & {$[108]$} \\
\hline S. lanata Crantz. (=S. germanica L. subsp. germanica) & Roots & Chlorogenic acid (103) & [82] \\
\hline S. tmolea Boiss & Aerial parts \# & $\begin{array}{l}\text { 4-Hydroxybenzoic acid (99), } \\
\text { Chlorogenic acid (103) }\end{array}$ & [85] \\
\hline S. thirkei K. Koch & Aerial parts\# & Chlorogenic acid (103) & [84] \\
\hline S. germanica L. subsp. salviifolia (Ten.) Gams. & Aerial parts & Arbutin (107) & [109] \\
\hline \multicolumn{4}{|c|}{ Section Olisia } \\
\hline S. atherocalyx C. Koch. & & $\begin{array}{c}\text { Neochlorogenic acid (105), } \\
\text { p-Coumaric acid (106), } \\
\text { Caffeic acid (108) }\end{array}$ & {$[110]$} \\
\hline S. recta $\mathrm{L}$. & Aerial parts \# & $\begin{array}{l}\text { 1-Caffeoylquinic acid (102), } \\
\text { Chlorogenic acid (103), } \\
\text { 4-Caffeoylquinic acid (104) }\end{array}$ & [14] \\
\hline \multicolumn{4}{|c|}{ Section Stachys } \\
\hline \multirow[t]{2}{*}{ S. palustris L. } & & $\begin{array}{l}\text { 1-Caffeoylquinic acid (102), } \\
\text { Chlorogenic acid (103), } \\
\text { 4-Caffeoylquinic acid (104), } \\
\text { Caffeic acid (108) }\end{array}$ & {$[104]$} \\
\hline & & $\begin{array}{l}\text { Cryptochlorogenic acid (104), } \\
\text { Neochlorogenic acid (105) }\end{array}$ & [23] \\
\hline \multicolumn{4}{|c|}{ Subgenus Betonica } \\
\hline \multicolumn{4}{|c|}{ Section Betonica } \\
\hline S. officinalis L. (=Betonica officinalis L.) & Leaves \# & Chlorogenic acid (103) & {$[111]$} \\
\hline
\end{tabular}

Table 8. Acetophenone glycosides from Stachys spp.

\begin{tabular}{cccc}
\hline Species & Plant Parts & Compound & Ref \\
\hline & Subgenus Stachys & \\
\hline & Section Eriostomum & \\
\hline $\begin{array}{c}\text { S. lanata Crantz. (=S. germanica L. } \\
\text { subsp. germanica) }\end{array}$ & Roots & $\begin{array}{c}\text { Androsin (109), Neolloydosin (110), } \\
\text { Glucoacetosyringone (111) }\end{array}$ & [82] \\
\hline
\end{tabular}

Table 9. Lignans from Stachys spp.

\begin{tabular}{cccc}
\hline Species & Plant Parts & Compound & Ref \\
\hline \multicolumn{4}{c}{ Subgenus Stachys } \\
\hline \multicolumn{4}{c}{ Section Stachys } \\
\hline S. mialhesii Noé & Aerial Parts & $(+)$-Sesamin $(\mathbf{1 1 2}),(+)$-Paulownin $(\mathbf{1 1 3})$ & {$[103]$} \\
\hline \multicolumn{4}{c}{ Section Olisia } \\
\hline S. tetragona Boiss. \& Heldr. & Aerial parts & (7S-8R)-Urolignoside (114) & {$[100]$} \\
\hline
\end{tabular}


Table 10. Phenylethanoid glycosides from Stachys spp.

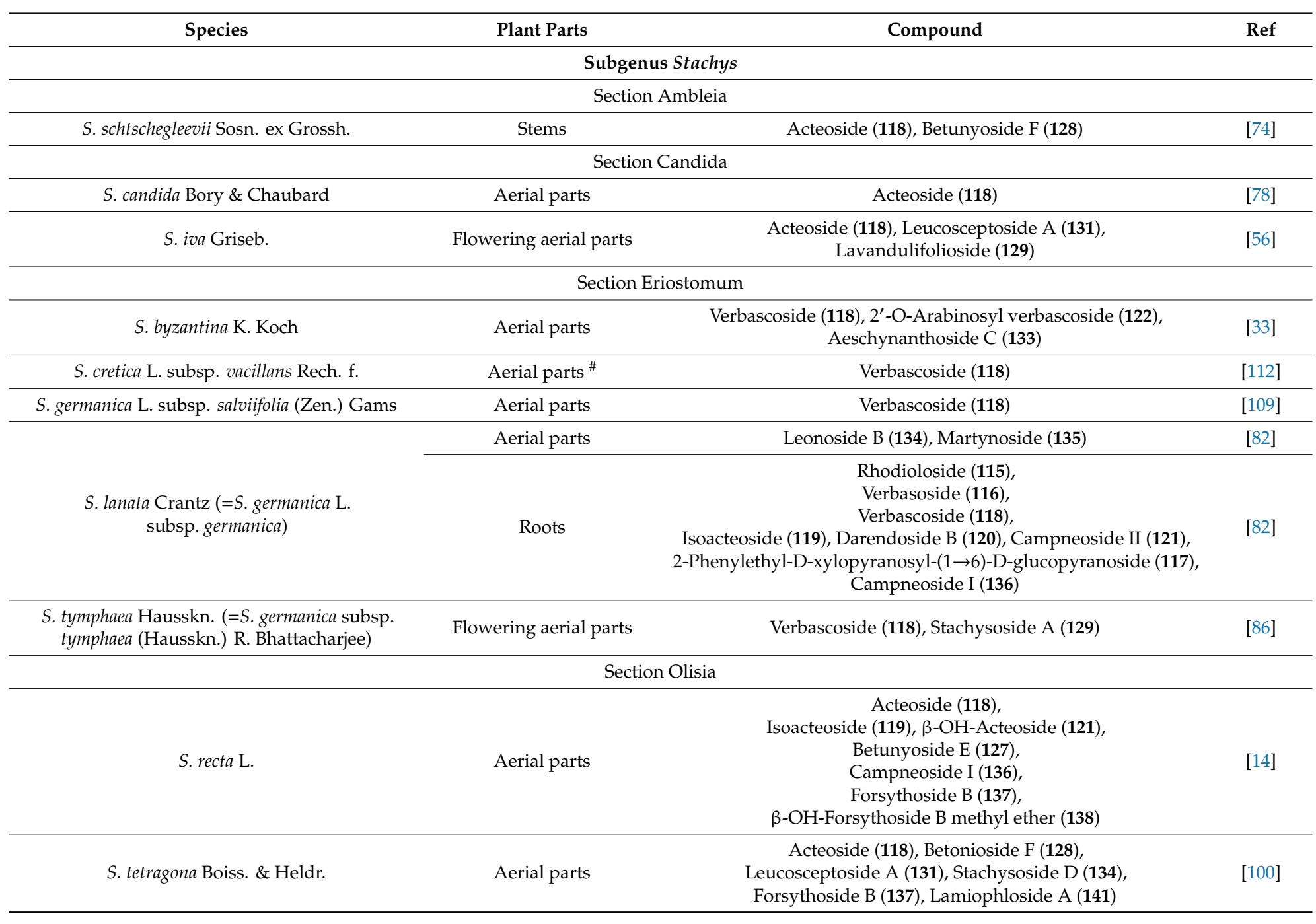


Table 10. Cont.

\begin{tabular}{|c|c|c|c|}
\hline Species & Plant Parts & Compound & Ref \\
\hline \multicolumn{4}{|c|}{ Subgenus Stachys } \\
\hline \multicolumn{4}{|c|}{ Section Stachys } \\
\hline S. affinis Bunge (=S. sieboldii Miq.) & & Stachysosides A (129), B (139), C (140) & [113] \\
\hline S. riederi Cham. & \multicolumn{2}{|c|}{ Section Zietenia } & \\
\hline \multirow[b]{2}{*}{ S. lavandulifolia Vahl } & Aerial parts & $\begin{array}{c}\text { Acteoside (118) } \\
\text { Lavandulifolioside (129) }\end{array}$ & [115] \\
\hline & Aerial parts & $\begin{array}{c}\text { Verbascoside (118), Lavandulofolioside A (129), } \\
\text { Lavandufolioside B (130), } \\
\text { Leucosceptoside A (131) }\end{array}$ & [12] \\
\hline $\begin{array}{l}\text { S. macrantha (C. Koch.) Stearn } \\
\text { (=Betonica grandiflora Willd.) }\end{array}$ & Aerial parts & $\begin{array}{l}\text { Verbascoside (118), } \\
\text { Leucosceptoside A (131), } \\
\text { Martynoside (135), } \\
\text { Lavandulifolioside (129) }\end{array}$ & [117] \\
\hline S. officinalis (L.) Trevis. (=Betonica officinalis L.) & Aerial parts & $\begin{array}{c}\text { Acteoside (118), } \\
\text { Acteoside isomer (isoacteoside) (119), Campneoside II (121), } \\
\text { Betonyosides A-F (123-128), } \\
\text { Leucosceptoside B (132), Forsythoside B (137) }\end{array}$ & [118] \\
\hline $\begin{array}{l}\text { S. alopecuros (L.) Benth subsp. divulsa } \\
\text { (Ten.) Grande }\end{array}$ & Flowering aerial parts & Verbascoside (118) & [119] \\
\hline \multicolumn{4}{|c|}{ Former Stachys species } \\
\hline $\begin{array}{l}\text { S. parviflora Benth. (=Phlomidoschema parviflorum } \\
\text { (Benth.) Vved.) }\end{array}$ & Whole plant & Parvifloroside A (142), Parvifloroside B (143) & [120] \\
\hline
\end{tabular}

\# identified compounds by means of HPLC, LC-MS, etc. 
Table 11. Phenylpropanoid glucosides from Stachys spp.

\begin{tabular}{ccccc}
\hline Species & Plant Parts & Compound & Ref \\
\hline \multicolumn{4}{c}{ Subgenus Stachys } \\
\hline \multicolumn{4}{c}{ Section Eriostomum } \\
\hline S. lanata Crantz. (=S. germanica L. subsp. germanica) & Roots & Coniferin (144), Syringin (145) & [82] \\
\hline
\end{tabular}

Table 12. Iridoids from Stachys spp.

\begin{tabular}{|c|c|c|c|}
\hline Species & Plant Parts & Compound & Ref \\
\hline \multicolumn{4}{|c|}{ Subgenus Stachys } \\
\hline \multicolumn{4}{|c|}{ Section Ambleia } \\
\hline S. inflata Benth. & & Ajugol (146), Ajugoside (147), & [121] \\
\hline \multicolumn{4}{|c|}{ Section Aucheriana } \\
\hline S. glutinosa L. & Aerial parts & $\begin{array}{c}\text { Harpagide (148), } \\
\text { Acetylharpagide (150), Monomelittoside (165), } \\
\text { Melittoside (166), } \\
\text { Allobetonicoside (161), } \\
\text { 5-Allosyloxy-aucubin (167) }\end{array}$ & [122] \\
\hline \multicolumn{4}{|c|}{ Section Campanistrum } \\
\hline $\begin{array}{l}\text { S. ocymastrum (L.) Briq. } \\
\text { (=S. hirta L.) }\end{array}$ & Leaves & $\begin{array}{c}\text { 6ß-Acetoxyipolamiide (172), } \\
\text { 6ß-Hydroxyipolamiide (173), Ipolamiide (174), } \\
\text { Ipolamiidoside (175), Lamiide (176) }\end{array}$ & [123] \\
\hline \multicolumn{4}{|c|}{ Section Candida } \\
\hline S. iva Griseb. & $\begin{array}{l}\text { Flowering Aerial } \\
\text { parts }\end{array}$ & $\begin{array}{c}\text { Harpagide (148), } \\
\text { 8-Acetylharpagide (150), } \\
\text { 8-Epi-loganic acid (157), } \\
\text { Gardoside (160), } \\
\text { 8-Epi-loganin (159), } \\
\text { Monomelittoside (165), } \\
\text { Melittoside (166) }\end{array}$ & {$[56]$} \\
\hline \multicolumn{4}{|c|}{ Section Corsica } \\
\hline S. corsica Pers. & & $\begin{array}{c}\text { Harpagide (148), } \\
\text { Acetylharpagide (150) }\end{array}$ & {$[79]$} \\
\hline \multicolumn{4}{|c|}{ Section Eriostomum } \\
\hline S. alpina L. & Stems, Leaves \# & $\begin{array}{c}\text { Ajugoside (147), } \\
\text { Harpagide (148), Acetylharpagide (150), } \\
\text { Harpagoside (154), Aucubin (164), Catalpol (163) }\end{array}$ & [124] \\
\hline S. balansae Boiss. \& Kotschy & & Ajugol (146), Ajugoside (147) & [125] \\
\hline \multirow[b]{2}{*}{ S. germanica L. } & & Harpagide (148) & [125] \\
\hline & $\begin{array}{c}\text { Leaf, } \\
\text { Inflorescence }\end{array}$ & $\begin{array}{c}\text { Ajugoside (147), } \\
\text { Harpagide (148), Acetylharpagide (150), } \\
\text { Harpagoside (154), Aucubin (164), Catalpol (163) }\end{array}$ & [124] \\
\hline S. spectabilis Choisy ex DC. & & Ajugol (146), Ajugoside (147), Harpagide (148) & [125] \\
\hline S. byzantina K. Koch. & Aerial parts $\#$ & $\begin{array}{c}\text { Ajugoside (147), } \\
\text { Harpagide (148), Acetylharpagide (150), } \\
\text { Harpagoside (154), Catalpol (163), } \\
\text { Aucubin (164) }\end{array}$ & [124] \\
\hline
\end{tabular}


Table 12. Cont.

\begin{tabular}{|c|c|c|c|}
\hline Species & Plant Parts & Compound & Ref \\
\hline \multicolumn{4}{|c|}{ Subgenus Stachys } \\
\hline \multicolumn{4}{|c|}{ Section Eriostomum } \\
\hline \multirow[b]{2}{*}{$\begin{array}{l}\text { S. germanica L. subsp. salviifolia } \\
\text { (Zen.) Gams }\end{array}$} & $\begin{array}{l}\text { Flowering Aerial } \\
\text { parts }\end{array}$ & Harpagide (148) & [86] \\
\hline & Aerial parts & $\begin{array}{c}\text { Ajugol (146), } \\
\text { Harpagide (148), } \\
\text { 7-Hydroxyharpagide (149), } \\
\text { 5-Allosyloxy-aucubin (167) }\end{array}$ & [109] \\
\hline \multirow{2}{*}{$\begin{array}{l}\text { S. lanata Crantz. (=S. germanica } \\
\text { L. subsp. germanica) }\end{array}$} & Roots & Stachysosides E (168), G (170), H (171) & [82] \\
\hline & Aerial parts & Stachysosides E (168), F (169) & [82] \\
\hline $\begin{array}{c}\text { S. tymphaea Hausskn. } \\
\text { (=S. germanica subsp. tymphaea } \\
\text { (Hausskn.) R. Bhattacharjee) }\end{array}$ & Aerial parts & Harpagide (148) & [86] \\
\hline \multicolumn{4}{|c|}{ Section Olisia } \\
\hline S. angustifolia M. Bieb. & & $\begin{array}{l}\text { Ajugoside (147), Acetylharpagide (150), } \\
\text { Harpagide (148), Melittoside (166) }\end{array}$ & [92] \\
\hline S. anпua (L.) L. & & $\begin{array}{l}\text { Ajugoside (147), } \\
\text { Acetylharpagide (150), } \\
\text { Melittoside (166) }\end{array}$ & [92] \\
\hline S. atherocalyx C. Koch. & & $\begin{array}{c}\text { Ajugol (146), Harpagide (148), Acetylharpagide } \\
\text { (150), } \\
\text { Melittoside (166) }\end{array}$ & $\begin{array}{l}{[92} \\
125]\end{array}$ \\
\hline S. beckeana Dörfl. \& Hayek & & $\begin{array}{c}\text { Harpagide (148), Ajugol (146), Acetylharpagide } \\
\text { (150), } \\
\text { Melittoside (166) }\end{array}$ & [92] \\
\hline S. iberica M. Bieb. & & $\begin{array}{c}\text { Ajugol (146), Ajugoside (147), Harpagide (148), } \\
\text { Acetylharpagide (150) }\end{array}$ & [121] \\
\hline \multirow{3}{*}{ S. recta $\mathrm{L}$. } & & $\begin{array}{c}\text { Ajugol (146), Harpagide (148), } \\
\text { Acetylharpagide (150), Melittoside (166) }\end{array}$ & [92] \\
\hline & Leaves & 8-Acetylharpagide (150), Melittoside ${ }^{\#}$ (166) & {$[14]$} \\
\hline & Aerial parts \# & $\begin{array}{c}\text { Ajugoside (147), Harpagide (148), } \\
\text { Acetylharpagide (150), } \\
\text { Harpagoside (154), Catalpol (163), } \\
\text { Aucubin (164) }\end{array}$ & [124] \\
\hline $\begin{array}{l}\text { S. baldaccii (Maly) Hand-Mazz } \\
\text { (=S. recta L. subsp. baldaccii (K. } \\
\text { Maly) Hayek) }\end{array}$ & & $\begin{array}{l}\text { Ajugol (146), Ajugoside (147), Harpagide (148), } \\
\text { Acetylharpagide (150), Melittoside (166) }\end{array}$ & [92] \\
\hline $\begin{array}{c}\text { S. subcrenata Vis. } \\
\text { (=S. recta subsp. subcrenata) }\end{array}$ & & $\begin{array}{c}\text { Ajugol (146), Harpagide (148), } \\
\text { Acetylharpagide (150), Melittoside (166) }\end{array}$ & [92] \\
\hline S. labiosa Bertol. & & $\begin{array}{l}\text { Ajugol (146), Harpagide (148), Acetylharpagide } \\
\text { (150), Melittoside (166) }\end{array}$ & [92] \\
\hline S. leucoglossa Griseb. & & $\begin{array}{c}\text { Ajugol (146), Harpagide (148), Acetylharpagide } \\
\text { (150), Melittoside (166) }\end{array}$ & [92] \\
\hline S. spinosa L. & Aerial parts & $\begin{array}{c}\text { Ajugol (146), Harpagide (148), } \\
\text { 7-O-Acetyl-8-epi-loganic acid (158) }\end{array}$ & [98] \\
\hline \multirow[t]{2}{*}{ S. tetragona Boiss. \& Heldr. } & & $\begin{array}{l}\text { Ajugol (146), Ajugoside (147), Harpagide (148), } \\
\text { Acetylharpagide (150), Melittoside (166) }\end{array}$ & [92] \\
\hline & Aerial parts & $\begin{array}{c}\text { 8-Acetyl-harpagide (150), } \\
\text { 5-O-Allopyranosyl-monomelittoside (167) }\end{array}$ & [100] \\
\hline
\end{tabular}


Table 12. Cont.

\begin{tabular}{|c|c|c|c|}
\hline Species & Plant Parts & Compound & Ref \\
\hline \multicolumn{4}{|c|}{ Subgenus Stachys } \\
\hline \multicolumn{4}{|c|}{ Section Stachys } \\
\hline $\begin{array}{l}\text { S. affinis Bunge } \\
\text { (= S. sieboldii Miq.) }\end{array}$ & Tubers & $\begin{array}{c}\text { Harpagide (148), Acetylharpagide (150), } \\
\text { Melittoside (166), 5-Allosyloxy-aucubin (167) }\end{array}$ & [27] \\
\hline S. palustris L. & Aerial parts \# & $\begin{array}{c}\text { Ajugoside (147), Harpagide (148), } \\
\text { Acetylharpagide (150), } \\
\text { Harpagoside (154), Catalpol (163), Aucubin (164) }\end{array}$ & {$[124$} \\
\hline S. sylvatica L. & Aerial parts \# & $\begin{array}{l}\text { Ajugoside (147), Harpagide (148), } \\
\text { Acetylharpagide (150), } \\
\text { Harpagoside (154), Catalpol (163), } \\
\text { Aucubin (164) }\end{array}$ & {$[124]$} \\
\hline \multicolumn{4}{|c|}{ Section Swainsoniana } \\
\hline S. anisochila Vis. \& Pancic & & $\begin{array}{l}\text { Acetylharpagide (150), } \\
\text { Melittoside (166) }\end{array}$ & [92] \\
\hline S. ionica Halácsy & & $\begin{array}{l}\text { 8-epi-loganic acid (157), } \\
\text { Gardoside (160) }\end{array}$ & [20] \\
\hline \multirow{2}{*}{$\begin{aligned} & \text { S. menthifolia Vis. } \\
(= & \text { S. grandiflora Host.) }\end{aligned}$} & & $\begin{array}{l}\text { Ajugol (146), Harpagide (148), } \\
\text { Acetylharpagide (150), } \\
\text { Melittoside (166) }\end{array}$ & [92] \\
\hline & Aerial parts \# & $\begin{array}{c}\text { Ajugoside (147) Harpagide (148), } \\
\text { Acetylharpagide (150), } \\
\text { Harpagoside (154), Catalpol (163), Aucubin (164) }\end{array}$ & {$[124$} \\
\hline \multicolumn{4}{|c|}{ Section Zietenia } \\
\hline \multirow{2}{*}{ S. lavandulifolia Vahl. } & & Ajugol (146), Ajugoside (147) & {$[125$} \\
\hline & Aerial parts & $\begin{array}{l}\text { Melittoside (166), Monomelittoside (165), } \\
\text { 5-O-Allopyranosyl-monomelittoside (167) }\end{array}$ & [12] \\
\hline \multicolumn{4}{|c|}{ Subgenus Betonica } \\
\hline \multicolumn{4}{|c|}{ Section Betonica } \\
\hline $\begin{array}{l}\text { S. alopecuros (L.) Benth subsp. } \\
\text { divulsa (Ten.) Grande }\end{array}$ & $\begin{array}{l}\text { Flowering } \\
\text { aerial parts }\end{array}$ & $\begin{array}{c}\text { Harpagide (148), Acetylharpagide (150), } \\
\text { 4'-O- } \beta \text {-D-galactopyranosyl-teuhircoside (162) }\end{array}$ & {$[119]$} \\
\hline $\begin{array}{l}\text { S. foliosa Rupr. (=S. betoniciflora } \\
\text { Rupr.; Betonica foliosa Rupr.) }\end{array}$ & & Harpagide (148), Acetylharpagide (150) & {$[126$} \\
\hline S. betonicaeflora Rupr. & & Harpagide (148), Acetylharpagide (150) & {$[126$} \\
\hline $\begin{array}{l}\text { S. macrantha (C. Koch.) Stearn } \\
\text { (=Betonica grandiflora Steph. } \\
\text { ex Willd.) }\end{array}$ & Aerial parts & $\begin{array}{c}\text { Ajugol (146), } \\
\text { Ajugoside (147), } \\
\text { Harpagide (148), } \\
\text { 8-O-Acetyl-harpagide (150), } \\
\text { Reptoside (153), } \\
\text { Macranthoside [=8-O- } \\
\text { Allobetonicoside (161) }\end{array}$ & {$[117]$} \\
\hline \multirow{3}{*}{$\begin{array}{l}\text { S. officinalis (L.) Trevis. } \\
\text { (=Betonica officinalis L.) }\end{array}$} & Aerial parts & $\begin{array}{l}\text { Acetylharpagide (150), } \\
\text { Reptoside (153), } \\
\text { 6-O-Acetylmioporoside (155), } \\
\text { Allobetonicoside (161) }\end{array}$ & {$[127]$} \\
\hline & & Harpagide (148), Acetylharpagide (150) & {$[128]$} \\
\hline & Aerial parts $\#$ & $\begin{array}{c}\text { Ajugoside (147), Harpagide (148), } \\
\text { Acetylharpagide (150), } \\
\text { Harpagoside (154), Catalpol (163), Aucubin (164) }\end{array}$ & {$[124]$} \\
\hline
\end{tabular}


Table 12. Cont.

\begin{tabular}{|c|c|c|c|}
\hline Species & Plant Parts & Compound & Ref \\
\hline \multicolumn{4}{|c|}{ Unknown Section } \\
\hline S. grandidentata Lindl. ** & Aerial parts & $\begin{array}{c}\text { Ajugol (146), Harpagide (148), } \\
\text { Acetylharpagide (150), 5-Desoxy-harpagide (151), } \\
\text { 5-Desoxy-8-acetyl-harpagide (152), } \\
\text { Monomelittoside (165), } \\
\text { Melittoside (166) }\end{array}$ & [129] \\
\hline
\end{tabular}

Table 13. Diterpenes from Stachys spp.

\begin{tabular}{|c|c|c|c|}
\hline Species & Plant Parts & Compound & Ref \\
\hline \multicolumn{4}{|c|}{ Subgenus Stachys } \\
\hline \multicolumn{4}{|c|}{ Section Ambleia } \\
\hline \multirow{5}{*}{ S. aegyptiaca Pers. } & & $\begin{array}{c}\text { Stachysolone (177), } \\
\text { 11a,18-Dihydroxy-ent-kaur-16-ene (210) }\end{array}$ & [130] \\
\hline & Aerial parts & $\begin{array}{c}\text { Stachysperoxide (189), Stachysolone (177), } \\
\text { 7,13-Diacetyl-stachysolone (180) }\end{array}$ & [131] \\
\hline & Aerial parts & $\begin{array}{l}\text { Stachaegyptin A-C (190-192), } \\
\text { Roseostachenone (184), } \\
\quad \text { Stachysolone (177), } \\
\text { 7,13-Diacetyl-stachysolone (180) }\end{array}$ & [106] \\
\hline & Aerial parts & Stachaegyptins D, E $(193,194)$ & [132] \\
\hline & Aerial parts & $\begin{array}{c}\text { Stachaegyptins A (190), F-H (195-197), } \\
\text { Stachysperoxide (189) }\end{array}$ & [133] \\
\hline S. inflata Benth. & & $\begin{array}{l}\text { Annuanone (181), Stachylone (182), } \\
\text { Stachone (183) }\end{array}$ & [134] \\
\hline \multicolumn{4}{|c|}{ Section Aucheriana } \\
\hline S. glutinosa L. & Aerial parts & $\begin{array}{c}\text { Roseostachenone (184), } \\
3 \alpha, 4 \alpha \text {-Epoxyroseostachenol (188) }\end{array}$ & [107] \\
\hline \multicolumn{4}{|c|}{ Section Eriostomum } \\
\hline S. balansae Boiss. \& Kotschy & & Annuanone (181), Stachylone (182) & [134] \\
\hline $\begin{array}{l}\text { S. lanata Crantz. (=S. germanica } \\
\text { L. subsp. germanica) }\end{array}$ & & $\begin{array}{c}\text { Ent-3 } \alpha \text {-acetoxy-kaur-16-en-19-oic acid (207), } \\
\text { Ent-3 } \alpha, 19 \text {-dihydroxy-kaur-16-ene (208), } \\
\text { Ent-3 } \alpha \text {-hydroxy-kaur-16-en-19-oic } \\
\text { acid (209) }\end{array}$ & [135] \\
\hline \multicolumn{4}{|c|}{ Section Mucronata } \\
\hline S. mucronata Sieb. & Aerial parts & $\begin{array}{c}\text { Ribenone [=3 } \beta \text {-hydroxy-13-epi-ent-manoyl } \\
\text { oxide] (198), Ribenol } \\
\text { [=3-keto-13-epi-ent-manoyl oxide] (199) }\end{array}$ & [57] \\
\hline \multicolumn{4}{|c|}{ Section Olisia } \\
\hline \multirow{2}{*}{ S. annua (L.) L. } & & Stachysolone (177) & {$[136,137]$} \\
\hline & & $\begin{array}{l}\text { Annuanone (181), Stachylone (182), } \\
\text { Stachone (183) }\end{array}$ & [138] \\
\hline
\end{tabular}


Table 13. Cont.

\begin{tabular}{|c|c|c|c|}
\hline Species & Plant Parts & Compound & Ref \\
\hline \multicolumn{4}{|c|}{ Subgenus Stachys } \\
\hline \multicolumn{4}{|c|}{ Section Olisia } \\
\hline S. atherocalyx C. Koch. & & $\begin{array}{l}\text { Annuanone (181), } \\
\text { Stachylone (182), } \\
\text { Stachone (183) }\end{array}$ & [134] \\
\hline S. distans Benth & Aerial parts & (+)-6-Deoxyandalusol (201) & [139] \\
\hline S. iberica M. Bieb. & & $\begin{array}{l}\text { Annuanone (181), } \\
\text { Stachylone (182), } \\
\text { Stachone (183) }\end{array}$ & [134] \\
\hline S. recta $\mathrm{L}$. & Aerial parts & $\begin{array}{c}\text { 7,13-Diacetate stachysolone (180), } \\
\text { 7-Acetate stachysolone (178), } \\
\text { 13-Acetate stachysolone (179) }\end{array}$ & [140] \\
\hline \multicolumn{4}{|c|}{ Section Roseostachys } \\
\hline S. rosea Boiss. & Aerial parts & $\begin{array}{c}\text { Roseostachenone (184), } \\
\text { Roseostachone (185), 13-epi-sclareol (200), } \\
\text { Roseostachenol (186), Roseotetrol (187) }\end{array}$ & [141] \\
\hline \multicolumn{4}{|c|}{ Section Stachys } \\
\hline S. mialhesii Noé & Aerial parts & Horminone (211) & [103] \\
\hline S. palustris L. & & Annuanone (181) & [134] \\
\hline \multirow{4}{*}{ S. sylvatica L. } & & Stachysic acid (204) & [142] \\
\hline & & $\begin{array}{l}\text { Annuanone (181), } \\
\text { Stachylone (182), } \\
\text { Stachone (183) }\end{array}$ & [134] \\
\hline & & $\begin{array}{c}\text { Stachysic acid (204), } \\
\text { 6ß-Hydroxy-ent-kaur-16-ene (205), } \\
\text { 6ß,18-Dihydroxy-ent-kaur-16-ene (206) }\end{array}$ & [142] \\
\hline & & Betolide (214) & [143] \\
\hline \multicolumn{4}{|c|}{ Section Swainsoniana } \\
\hline S. ionica Halácsy & Aerial parts & (+)-6-Deoxyandalusol (201) & [139] \\
\hline S. plumosa Griseb. & Aerial parts & $\begin{array}{c}\text { (+)-6-Deoxyandalusol (201), } \\
\text { 13-Epi-jabugodiol (202), } \\
\text { (+)-Plumosol (203) }\end{array}$ & [144] \\
\hline \multicolumn{4}{|c|}{ Section Zietenia } \\
\hline S. lavandulifolia Vahl. & Aerial parts & Stachysolone (177) & [116] \\
\hline \multicolumn{4}{|c|}{ Subgenus Betonica } \\
\hline \multicolumn{4}{|c|}{ Section Betonica } \\
\hline \multirow{3}{*}{$\begin{array}{l}\text { S. officinalis (L.) Trevis. } \\
\text { (=Betonica officinalis L.) }\end{array}$} & & Betolide (214) & [145] \\
\hline & & $\begin{array}{c}\text { Betonicolide (215), } \\
\text { Betonicosides A-D (216-219) }\end{array}$ & [145] \\
\hline & Roots & Betolide (214) & [143] \\
\hline $\begin{array}{l}\text { S. scardica (Griseb.) Hayek } \\
\text { (=Betonica scardica Griseb.) }\end{array}$ & Roots & Betolide (214) & [143] \\
\hline \multicolumn{4}{|c|}{ Former Stachys species } \\
\hline $\begin{array}{c}\text { S. parviflora Benth. } \\
\text { (=Phlomidoschema parviflorum } \\
\text { (Benth.) Vved.) }\end{array}$ & Whole plant & $\begin{array}{l}\text { Stachyrosane } 1 \text { (212) } \\
\text { Stachyrosane } 2 \text { (213) }\end{array}$ & [133] \\
\hline
\end{tabular}


Table 14. Triterpene derivatives, Phytosterols and Phytoecdysteroids from Stachys spp.

\begin{tabular}{|c|c|c|c|}
\hline Species & Plant Parts & Compound & Ref \\
\hline \multicolumn{4}{|c|}{ Subgenus Stachys } \\
\hline \multicolumn{4}{|c|}{ Section Eriostomum } \\
\hline \multirow[b]{2}{*}{ S. byzantina K. Koch } & Aerial parts & Stigmasterol (220), & [17] \\
\hline & & $\begin{array}{l}\text { } \beta \text {-Sitosterol (221), Lawsaritol (223), } \\
\text { Stigmastan-3,5-dien-7-one (224) }\end{array}$ & [35] \\
\hline S. hissarica Regel & - & $\begin{array}{l}\text { 20-Hydroxyecdysone (239), Polipodin B (240), } \\
\text { Integristeron A (241), } \\
\text { 2-Desoxy-20-hydroxyecdysone (242), } \\
\text { 2-Desoxyecdyson (243) }\end{array}$ & [67] \\
\hline \multicolumn{4}{|c|}{ Section Olisia } \\
\hline S. annua (L.) L. & Aerial parts & $\beta$-Sitosterol (221), Ursolic acid (226) & [95] \\
\hline S. spinosa L. & Aerial parts & $\begin{array}{c}\text { Stigmasterol (220), } \\
\beta \text {-Sitosterol (221), Oleanolic acid (227), } \\
12 \alpha \text {-Hydroxy-oleanolic lactone (228) }\end{array}$ & [99] \\
\hline S. tetragona Boiss. \& Heldr. & Aerial parts & $\begin{array}{c}\text { Stigmasterol (220), } \\
\beta \text {-Sitosterol (221), } \\
\text { Oleanolic acid (227), }\end{array}$ & [100] \\
\hline \multicolumn{4}{|c|}{ Section Stachys } \\
\hline S. palustris L. & & $\begin{array}{l}\beta \text {-Sitosterol (221), } \\
\alpha \text {-amyrin (225) }\end{array}$ & [146] \\
\hline S. riederi Cham. & Whole plant & Stachyssaponins I-VIII (231-238) & [147] \\
\hline \multicolumn{4}{|c|}{ Subgenus Betonica } \\
\hline \multicolumn{4}{|c|}{ Section Betonica } \\
\hline $\begin{array}{l}\text { S. alopecuros (L.) Benth subsp. } \\
\text { divulsa (Ten.) Grande }\end{array}$ & $\begin{array}{l}\text { Flowering } \\
\text { aerial parts }\end{array}$ & 3-O- $\beta$-Sitosterol-glucoside (222) & [119] \\
\hline \multicolumn{4}{|c|}{ Former Stachys species } \\
\hline $\begin{array}{l}\text { S. parviflora Benth. } \\
\text { (=Phlomidoschema parviflorum } \\
\text { (Benth.) Vved.) }\end{array}$ & Aerial parts & $\begin{array}{l}\text { Stachyssaponin A (229), } \\
\text { Stachyssaponin B (230) }\end{array}$ & [63] \\
\hline
\end{tabular}

Table 15. Megastigmane derivatives from Stachys spp.

\begin{tabular}{cccc}
\hline Species & Plant Parts & Compound & Ref \\
\hline \multicolumn{2}{c}{ Subgenus Stachys } \\
\hline \multicolumn{3}{c}{ Section Eriostomum } \\
S. byzantina K. Koch. & Aerial parts & $\begin{array}{c}\text { Byzantionoside B (245), Icariside B2 (246), } \\
\text { (6R, 9R)- and (6R, 9S)-3-oxo- } \alpha \text {-ionol } \\
\text { glucosides (247), Blumeol C glucoside (248) }\end{array}$ & [148] \\
\hline \multirow{2}{*}{$\begin{array}{c}\text { S. lanata Crantz (=S. germanica } \\
\text { L. subsp. germanica) }\end{array}$} & Aerial parts & Vomifoliol (249), Dehydrovomifoliol (250) & [82] \\
\cline { 2 - 4 } & Roots & Citroside A (251) & [82] \\
\hline
\end{tabular}

\subsection{Flavonoids}

The genus Stachys consists a rich source of flavonoids. Accumulating studies have reported the several types of flavonoids occurring in Stachys spp., including flavones (Tables 2 and 16), poly-methylated flavones (Tables 3 and 17), flavonols (Tables 4 and 18), flavanones (Tables 5 and 19) and one biflavonoid (Tables 6 and 20).

Regarding the flavone derivatives (Tables 2 and 16), 18 flavone 7-O-acetylallosylglucosides were mentioned in the most species of subgenus Stachys (31 species). The flavone 7-O-glucosides were 
also found in many species through the two subgenera. Marin et al. (2004) reported that tricetin $3^{\prime}, 4^{\prime}, 5^{\prime}$-trimethyl-7-O-glucoside (62) consists a chemotaxonomic marker for the subgenus Betonica [5]. Precisely, selgin 7-O-glucoside (59), tricin 7-O-glucoside (61) and tricetin $3^{\prime}, 4^{\prime}, 5^{\prime}$-trimethyl-7-Oglucoside (62) were identified from the leaves of three species of the latter subgenus; $S$. alopecuros (section Betonica), S. officinalis (section Betonica) and S. scardica (section Macrostachya) [5]. Furthermore, derivatives of apigenin $p$-coumaroyl glucosides and chrysoeriol $p$-coumaroyl glucosides were reported in Stachys species, though some $p$-coumaroyl glucosides (not determined) were also identified $[5,75]$. To be mentioned that chrysoeriol 7-O-glucoside $(43)$, chrysoeriol $p$-coumaroyl glucosides $(46,47)$ and chrysoeriol 7-O-[6"' -O-acetyl-allosyl]-(1 $\rightarrow 2)$-glucoside (stachyspinoside) (44) were mainly isolated from wild Greek taxa of the subgenus Stachys $[3,77,98,99,102]$, apart from the Greek species $S$. ionica [20], S. tetragona [100] and the cultivated species S. iva [56]. Nazemiyeh et al. (2006) investigated the phytochemical profile of the stems of $S$. schtschegleevii, reporting four flavonoids, among them were also two $p$-coumaroyl derivatives of apigenin and chrysoeriol [74]. Moreover, flavone 7-O-mannosylglucosides were reported from the two species S. atherocalyx (section Eriostomum) and S. spectabilis (section Olisia) $[72,89,90]$. Few flavone C-glucosides were mentioned in the species $S$. aegyptiaca (subg. Stachys; sect. Ambleia), S. officinalis (subg. Betonica; sect. Betonica), and S. scardica (subg. Betonica; sect. Macrostachya) $[5,68,104]$. Zinchenko (1973) reported the existence of two derivatives of methoxybaicalein, namely palustrin (63) and palustrinoside (64), from the species S. palustris of subgenus Stachys (section Stachys) [104]. Notably, the subterranean organs of S. annua were investigated and the isolation of two flavone derivatives was reported, namely $4^{\prime}$-O-methyl-isoscutellarein (12) and 4'-O-methyl-isoscutellarein-7-O-(6"'-O-acetyl)allopyranosyl-(1 $\rightarrow 2)$-glucopyranoside (21) [95].

Furthermore, our survey revealed the presence of poly-methylated flavones in the genus Stachys (Tables 3 and 17). Precisely, six species and four subspecies from subgenus Stachys, as well as one species from subgenus Betonica, are found to contain poly-methylated flavones. The most common representative was xanthomicrol (69) which was mentioned in seven Stachys species and subspecies of different sections from the subgenus Stachys $[20,68,74,77,78,102,107]$. In the stems of the species S. schtschegleevii, apart from xanthomicrol (69), was also found circimaritin (66) [74].

A few studies mentioned the existence of flavonols in Stachys spp. (Tables 4 and 18), mainly in species occurred in Greece. Afouxenidi and colleagues (2018) isolated kaempferol (91) from the $n$-butanol residue of the aerial parts of S. tetragona [100], which was also identified in the aerial parts of S. cretica subsp. smyrnaea [81]. Moreover, isorhamnetin (92) was isolated from the methanol extract of the aerial parts of S. swainsonii subsp. swainsonii and S. swainsonii subsp. argolica [102]. A study conducted by Marin et al. (2004) identified the presence of quercetin 3-O-rutinoside (93) and isorhamnetin 3-O-glucoside (94) from the aerial parts of S. palustris [5].

In addition, three flavanones were isolated from three species of the genus Stachys (Tables 5 and 19). Eriodictyol (95) was mentioned in S. cretica [108] and in one subspecies of S. swainsonii [102], while naringenin (96) was isolated from the aerial parts of the species S. aegyptiaca [104]. A flavanone rutinoside, known as hesperidin (97), was identified as one of the major compounds of the aerial parts of $S$. cretica subsp. smyrnaea [81].

Of great interest is the isolation of a rare diflavone ester of $\mu$-truxinic acid, namely stachysetin (98). It is well-known that diglycoside flavone esters of dicarboxylic acids are rare compounds in plant kingdom. Stachysetin was firstly isolated from the ethanol extract $(70 \% \mathrm{v} / \mathrm{v})$ of the aerial parts of S. aegyptiaca [69]. Then, Murata and co-workers (2008) reported it in the methanol residue $(80 \% \mathrm{v} / \mathrm{v})$ of the aerial parts of S. lanata [82]. In a current study carried out by Pritsas et al. (2020), stachysetin was isolated from the methanol: aqueous (5:1) extract from the flowering aerial parts of the cultivated $S$. iva (Tables 6 and 20) [56]. Up to now, there is no report of this secondary metabolite in the species of the subgenus Betonica. The presence of this rare natural compound in the sections Ambleia, Eriostomum and Candida of the subgenus Stachys might be considered as a chemotaxonomic marker among the two subgenera and of the genus Stachys. 
Table 16. Chemical structures of flavones isolated from Stachys spp.

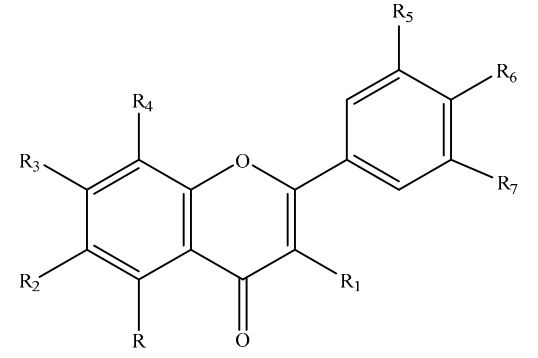

\begin{tabular}{|c|c|c|c|c|c|c|c|}
\hline Name & $\mathbf{R}_{1}$ & $\mathbf{R}_{\mathbf{2}}$ & $\mathbf{R}_{3}$ & $\mathbf{R}_{4}$ & $\mathbf{R}_{5}$ & $\mathbf{R}_{6}$ & $\mathbf{R}_{7}$ \\
\hline \multicolumn{8}{|c|}{$\mathrm{R}=\mathrm{OH}$} \\
\hline Apigenin (1) & $\mathrm{H}$ & $\mathrm{H}$ & $\mathrm{OH}$ & $\mathrm{H}$ & $\mathrm{H}$ & $\mathrm{OH}$ & $\mathrm{H}$ \\
\hline Apigenin 7-O- $\beta$-D-glucoside (cosmoside) (2) & $\mathrm{H}$ & $\mathrm{H}$ & O-glc & $\mathrm{H}$ & $\mathrm{H}$ & $\mathrm{OH}$ & $\mathrm{H}$ \\
\hline Apigenin 7-O-[6"'"-O-acetyl]- $\beta$-D-allosyl-(1 $\rightarrow 2)-\beta$-D-glucoside (3) & $\mathrm{H}$ & $\mathrm{H}$ & 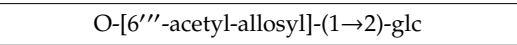 & $\mathrm{H}$ & $\mathrm{H}$ & $\mathrm{OH}$ & $\mathrm{H}$ \\
\hline Apigenin 7-(3"-E-p-coumaroyl)- $\beta$-D-glucoside (4) & $\mathrm{H}$ & $\mathrm{H}$ & O-(3"-E-p-coumaroyl)-glc & $\mathrm{H}$ & $\mathrm{H}$ & $\mathrm{OH}$ & $\mathrm{H}$ \\
\hline Apigenin 7-(3"-Z-p-coumaroyl)- $\beta$-D-glucoside (5) & $\mathrm{H}$ & $\mathrm{H}$ & O-(3"-Z-p-coumaroyl)-glc & $\mathrm{H}$ & $\mathrm{H}$ & $\mathrm{OH}$ & $\mathrm{H}$ \\
\hline Apigenin 7-(6"-E-p-coumaroyl)- $\beta$-D-glucoside (6) & $\mathrm{H}$ & $\mathrm{H}$ & $\mathrm{O}-\left(6^{\prime \prime}-\mathrm{E}-\mathrm{p}\right.$-coumaroyl)-glc & $\mathrm{H}$ & $\mathrm{H}$ & $\mathrm{OH}$ & $\mathrm{H}$ \\
\hline Apigenin 7-(6"-Z-p-coumaroyl)- $\beta$-D-glucoside (7) & $\mathrm{H}$ & $\mathrm{H}$ & $\mathrm{O}-\left(6^{\prime \prime}-\mathrm{Z}-\mathrm{p}\right.$-coumaroyl)-glc & $\mathrm{H}$ & $\mathrm{H}$ & $\mathrm{OH}$ & $\mathrm{H}$ \\
\hline Apigenin 7-(3", $6^{\prime \prime}-p$-dicoumaroyl)- $\beta$-D-glucoside (Anisofolin A) (8) & $\mathrm{H}$ & $\mathrm{H}$ & O-(3", $6^{\prime \prime}-p$-dicoumaroyl)-glc & $\mathrm{H}$ & $\mathrm{H}$ & $\mathrm{OH}$ & $\mathrm{H}$ \\
\hline Apigenin 8-C-glucoside (9) & $\mathrm{H}$ & $\mathrm{H}$ & $\mathrm{OH}$ & C-glc & $\mathrm{H}$ & $\mathrm{OH}$ & $\mathrm{H}$ \\
\hline Apigenin 6,8-di-C-glucoside (Vicenin-2) (10) & $\mathrm{H}$ & C-glc & $\mathrm{OH}$ & C-glc & $\mathrm{H}$ & $\mathrm{OH}$ & $\mathrm{H}$ \\
\hline Isoscutellarein (11) & $\mathrm{H}$ & $\mathrm{H}$ & $\mathrm{OH}$ & $\mathrm{OH}$ & $\mathrm{H}$ & $\mathrm{OH}$ & $\mathrm{H}$ \\
\hline Isoscutellarein 7-O-glucoside (11a) & $\mathrm{H}$ & $\mathrm{H}$ & O-glc & $\mathrm{OH}$ & $\mathrm{H}$ & $\mathrm{OH}$ & $\mathrm{H}$ \\
\hline 4'-Methyl-isoscutellarein (12) & $\mathrm{H}$ & $\mathrm{H}$ & $\mathrm{OH}$ & $\mathrm{OH}$ & $\mathrm{H}$ & $\mathrm{OCH}_{3}$ & $\mathrm{H}$ \\
\hline Isoscutellarein 7-O-allosyl-(1 $\rightarrow$ 2)-glucoside (13) & $\mathrm{H}$ & $\mathrm{H}$ & 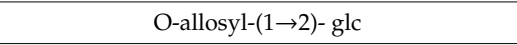 & $\mathrm{OH}$ & $\mathrm{H}$ & $\mathrm{OH}$ & $\mathrm{H}$ \\
\hline 3'-Hydroxy-isoscutellarein-7-O-[6"'-O-acetyl]- $\beta$-D-glucoside (14) & $\mathrm{H}$ & $\mathrm{H}$ & O-[6"'"-O-acetyl]- glc & $\mathrm{OH}$ & $\mathrm{OH}$ & $\mathrm{OH}$ & $\mathrm{H}$ \\
\hline Isoscutellarein 7-O-[6"'-O-acetyl]- $\beta$-D-allosyl-( $(\rightarrow 2)-\beta$-D-glucoside (15) & $\mathrm{H}$ & $\mathrm{H}$ & 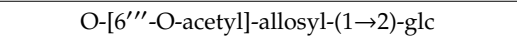 & $\mathrm{OH}$ & $\mathrm{H}$ & $\mathrm{OH}$ & $\mathrm{H}$ \\
\hline 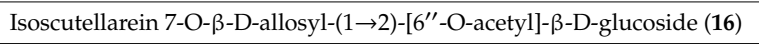 & $\mathrm{H}$ & $\mathrm{H}$ & 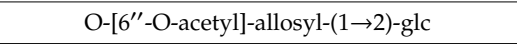 & $\mathrm{OH}$ & $\mathrm{H}$ & $\mathrm{OH}$ & $\mathrm{H}$ \\
\hline $\begin{array}{c}\text { Isoscutellarein 7-O-[6"' -O-acetyl]- } \beta \text {-D-allosyl-(1 } \rightarrow 2)-\left[6^{\prime \prime} \text {-O-acetyl]- }\right. \\
\beta \text {-D-glucoside (17) }\end{array}$ & $\mathrm{H}$ & $\mathrm{H}$ & 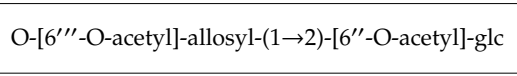 & $\mathrm{OH}$ & $\mathrm{H}$ & $\mathrm{OH}$ & $\mathrm{H}$ \\
\hline Isoscutellarein 8-O-(6"'-trans- - -coumaroyl)- $\beta$-D-glucoside (18) & $\mathrm{H}$ & $\mathrm{H}$ & $\mathrm{OH}$ & $\mathrm{O}$-(6"-trans-p-coumaroyl)-glc & $\mathrm{H}$ & $\mathrm{OH}$ & $\mathrm{H}$ \\
\hline $4^{\prime}$-Methyl-isoscutellarein 7-O- $\beta$-D-allosyl-( $(\rightarrow 2)-\beta$-D-glucoside (19) & $\mathrm{H}$ & $\mathrm{H}$ & O-allosyl-(1 $(\rightarrow 2)$-glc & $\mathrm{OH}$ & $\mathrm{H}$ & $\mathrm{OCH}_{3}$ & $\mathrm{H}$ \\
\hline
\end{tabular}


Table 16. Cont.

\begin{tabular}{|c|c|c|c|c|c|c|c|}
\hline Name & $\mathbf{R}_{1}$ & $\mathbf{R}_{2}$ & $\mathbf{R}_{3}$ & $\mathbf{R}_{4}$ & $\mathbf{R}_{5}$ & $\mathbf{R}_{6}$ & $\mathbf{R}_{7}$ \\
\hline \multicolumn{8}{|c|}{$\mathrm{R}=\mathrm{OH}$} \\
\hline 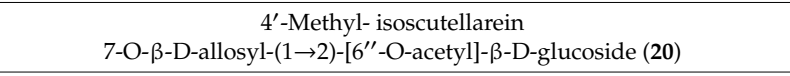 & $\mathrm{H}$ & $\mathrm{H}$ & 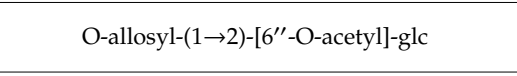 & $\mathrm{OH}$ & $\mathrm{H}$ & $\mathrm{OCH}_{3}$ & $\mathrm{H}$ \\
\hline $\begin{array}{c}4^{\prime}-\text { Methyl-isoscutellarein } \\
\text { 7-O- } \beta \text {-D-[6"' } \\
\end{array}$ & $\mathrm{H}$ & $\mathrm{H}$ & O-[6'"'-O-acetyl]-allosyl-(1 $\rightarrow 2)$-glc & $\mathrm{OH}$ & $\mathrm{H}$ & $\mathrm{OCH}_{3}$ & $\mathrm{H}$ \\
\hline $\begin{array}{c}\text { 4'-Methyl-isoscutellarein 7-O- } \\
\text { [2"-O-acetyl]- } \beta \text {-D-allosyl-( }(1 \rightarrow 2) \text { - } \beta \text {-D-glucoside (22) }\end{array}$ & $\mathrm{H}$ & $\mathrm{H}$ & 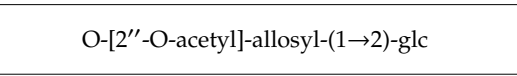 & $\mathrm{OH}$ & $\mathrm{H}$ & $\mathrm{OCH}_{3}$ & $\mathrm{H}$ \\
\hline $\begin{array}{c}4^{\prime} \text {-Methyl-isoscutellarein } \\
\text { 7-O- } \beta-D-\left[4^{\prime \prime \prime}-\mathrm{O}-\text { acetyl]-allosyl]-( }(\rightarrow 2)-\beta-D-\text { glucoside (annuoside) (23) }\right.\end{array}$ & $\mathrm{H}$ & $\mathrm{H}$ & 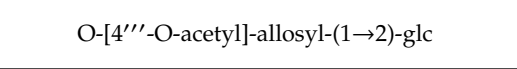 & $\mathrm{OH}$ & $\mathrm{H}$ & $\mathrm{OCH}_{3}$ & $\mathrm{H}$ \\
\hline $\begin{array}{c}4^{\prime} \text {-Methyl-isoscutellarein } \\
\text { 7-O-[6"' -O-acetyl]-allosyl-( }(1 \rightarrow 2)-\left[6^{\prime \prime} \text {-O-acety]]-glucoside (24) }\right.\end{array}$ & $\mathrm{H}$ & $\mathrm{H}$ & 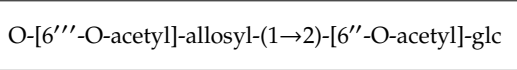 & $\mathrm{OH}$ & $\mathrm{H}$ & $\mathrm{OCH}_{3}$ & $\mathrm{H}$ \\
\hline Isostachyflaside (25) & $\mathrm{H}$ & $\mathrm{H}$ & $\mathrm{OH}$ & $\mathrm{OH}$ & $\mathrm{H}$ & O-mannosyl- $(1 \rightarrow 2)$-glc & $\mathrm{H}$ \\
\hline Acetyl-isostachyflaside (26) & $\mathrm{H}$ & $\mathrm{H}$ & $\mathrm{OH}$ & $\mathrm{OH}$ & $\mathrm{H}$ & O-[acetyl]-mannosyl- $(1 \rightarrow 2)$-glc & $\mathrm{H}$ \\
\hline Di-acetyl- isostachyflaside (27) & $\mathrm{H}$ & $\mathrm{H}$ & $\mathrm{OH}$ & $\mathrm{OH}$ & $\mathrm{H}$ & O-[diacetyl-mannosyl]- $(1 \rightarrow 2)$-glc & $\mathrm{H}$ \\
\hline Spectabiflaside (28) & $\mathrm{H}$ & $\mathrm{H}$ & O-mannosyl- (1 $\rightarrow 2)$-glc & $\mathrm{OH}$ & $\mathrm{OCH}_{3}$ & $\mathrm{OH}$ & $\mathrm{H}$ \\
\hline Scutellarein (29) & $\mathrm{H}$ & $\mathrm{OH}$ & $\mathrm{OH}$ & $\mathrm{H}$ & $\mathrm{H}$ & $\mathrm{OH}$ & $\mathrm{H}$ \\
\hline $\begin{array}{c}\text { Scutellarein 7-O- } \beta \text {-D-glucoside[5,6, } \\
4^{\prime} \text {-trihydroxyflavone-7-O- } \beta \text {-D-glucoside] (30) }\end{array}$ & $\mathrm{H}$ & $\mathrm{OH}$ & O-glc & $\mathrm{H}$ & $\mathrm{H}$ & $\mathrm{OH}$ & $\mathrm{H}$ \\
\hline Scutellarein 7-O- $\beta$-D-mannnosyl- (1 $\rightarrow 2)$ - $\beta$-D-glucoside (stachyflaside) (31) & $\mathrm{H}$ & $\mathrm{OH}$ & O-mannosyl- (1 $\rightarrow 2)$-glc & $\mathrm{H}$ & $\mathrm{H}$ & $\mathrm{OH}$ & $\mathrm{H}$ \\
\hline $\begin{array}{c}\text { 7-O- } \beta \text {-D-glucopyranosyl-5,6-dihydroxy-4'-methoxyflavone } \\
\text { (Stachannin A) (32) }\end{array}$ & $\mathrm{H}$ & $\mathrm{OH}$ & O-glc & $\mathrm{H}$ & $\mathrm{H}$ & $\mathrm{OCH}_{3}$ & $\mathrm{H}$ \\
\hline 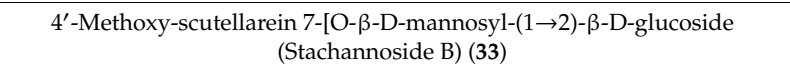 & $\mathrm{H}$ & $\mathrm{OH}$ & O-mannosyl- $(1 \rightarrow 2)$-glc & $\mathrm{H}$ & $\mathrm{H}$ & $\mathrm{OCH}_{3}$ & $\mathrm{H}$ \\
\hline Luteolin (34) & $\mathrm{H}$ & $\mathrm{H}$ & $\mathrm{OH}$ & $\mathrm{H}$ & $\mathrm{OH}$ & $\mathrm{OH}$ & $\mathrm{H}$ \\
\hline Luteolin 7-methyl ether (35) & $\mathrm{H}$ & $\mathrm{H}$ & $\mathrm{OCH}_{3}$ & $\mathrm{H}$ & $\mathrm{OH}$ & $\mathrm{OH}$ & $\mathrm{H}$ \\
\hline Luteolin 7-O- $\beta$-D-glucuronide (36) & $\mathrm{H}$ & $\mathrm{H}$ & O-glcA & $\mathrm{H}$ & $\mathrm{OH}$ & $\mathrm{OH}$ & $\mathrm{H}$ \\
\hline Luteolin 7-O- $\beta$-D-glucoside (37) & $\mathrm{H}$ & $\mathrm{H}$ & O-glc & $\mathrm{H}$ & $\mathrm{OH}$ & $\mathrm{OH}$ & $\mathrm{H}$ \\
\hline Luteolin 6-C-glucoside (isoorientin) (38) & $\mathrm{H}$ & -C-glc & $\mathrm{OH}$ & $\mathrm{H}$ & $\mathrm{OH}$ & $\mathrm{OH}$ & $\mathrm{H}$ \\
\hline Luteolin 7-O-[6"''-O-acetyl]-allosyl-(1 $\rightarrow 2$ )-glucoside (39) & $\mathrm{H}$ & $\mathrm{H}$ & 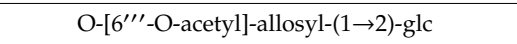 & $\mathrm{H}$ & $\mathrm{OH}$ & $\mathrm{OH}$ & $\mathrm{H}$ \\
\hline 6,8 Di-C- $\beta$-D-glucopyranosyl luteolin (Lucenin-2) (40) & $\mathrm{H}$ & C-glc & $\mathrm{OH}$ & C-glc & $\mathrm{OH}$ & $\mathrm{OH}$ & $\mathrm{H}$ \\
\hline $3^{\prime}, 4^{\prime}$-Dimethyl-luteolin-7-O- $\beta$-D-glucoside (41) & $\mathrm{H}$ & $\mathrm{H}$ & O-glc & $\mathrm{H}$ & $\mathrm{OCH}_{3}$ & $\mathrm{OCH}_{3}$ & $\mathrm{H}$ \\
\hline Chrysoeriol (42) & $\mathrm{H}$ & $\mathrm{H}$ & $\mathrm{OH}$ & $\mathrm{H}$ & $\mathrm{OCH}_{3}$ & $\mathrm{OH}$ & $\mathrm{H}$ \\
\hline Chrysoeriol 7-O- $\beta$-D-glucoside (43) & $\mathrm{H}$ & $\mathrm{H}$ & O-glc & $\mathrm{H}$ & $\mathrm{OCH}_{3}$ & $\mathrm{OH}$ & $\mathrm{H}$ \\
\hline
\end{tabular}


Table 16. Cont.

\begin{tabular}{|c|c|c|c|c|c|c|c|}
\hline Name & $\mathbf{R}_{1}$ & $\mathbf{R}_{2}$ & $\mathbf{R}_{3}$ & $\mathbf{R}_{4}$ & $\mathbf{R}_{5}$ & $\mathbf{R}_{6}$ & $\mathbf{R}_{7}$ \\
\hline \multicolumn{8}{|c|}{$\mathrm{R}=\mathrm{OH}$} \\
\hline $\begin{array}{c}\text { Chrysoeriol 7-O-[6"'"-O-acetyl]- } \beta \text {-D-allosyl-(1 } \rightarrow 2 \text { )-glucoside } \\
\text { (Stachyspinoside) (44) }\end{array}$ & $\mathrm{H}$ & $\mathrm{H}$ & O-[6"' ${ }^{\prime \prime \prime}$-O-acetyl]- allosyl-(1 $\left.\rightarrow 2\right)$-glc & $\mathrm{H}$ & $\mathrm{OCH}_{3}$ & $\mathrm{OH}$ & $\mathrm{H}$ \\
\hline $\begin{array}{c}\text { Chrysoeriol 7-O-[6"-O-acetyl]- } \beta \text {-D-allosyl-(1 } \rightarrow 2 \text { )-glucoside } \\
\text { (Isostachyspinoside) (45) }\end{array}$ & $\mathrm{H}$ & $\mathrm{H}$ & O-[6"-O-acetyl]- allosyl-(1 $\rightarrow 2)$-glc & $\mathrm{H}$ & $\mathrm{OCH}_{3}$ & $\mathrm{OH}$ & $\mathrm{H}$ \\
\hline Chrysoeriol 7-(3"-E- - -coumaroyl)- $\beta$-D-glucoside (46) & $\mathrm{H}$ & $\mathrm{H}$ & O-(3"-E-p-coumaroyl)-glc & $\mathrm{H}$ & $\mathrm{OCH}_{3}$ & $\mathrm{OH}$ & $\mathrm{H}$ \\
\hline Chrysoeriol 7-(6"-E- $p$-coumaroyl)- $\beta$-D-glucoside (47) & $\mathrm{H}$ & $\mathrm{H}$ & O-(6"'-E-p-coumaroyl)-glc & $\mathrm{H}$ & $\mathrm{OCH}_{3}$ & $\mathrm{OH}$ & $\mathrm{H}$ \\
\hline Hypolaetin (48) & $\mathrm{H}$ & $\mathrm{H}$ & $\mathrm{OH}$ & $\mathrm{OH}$ & $\mathrm{OH}$ & $\mathrm{OH}$ & $\mathrm{H}$ \\
\hline Hypolaetin-7-O-glucoside (49) & $\mathrm{H}$ & $\mathrm{H}$ & O-glc & $\mathrm{OH}$ & $\mathrm{OH}$ & $\mathrm{OH}$ & $\mathrm{H}$ \\
\hline Hypolaetin-7-O-glucuronide (49a) & $\mathrm{H}$ & $\mathrm{H}$ & O-glcA & $\mathrm{OH}$ & $\mathrm{OH}$ & $\mathrm{OH}$ & $\mathrm{H}$ \\
\hline 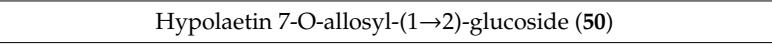 & $\mathrm{H}$ & $\mathrm{H}$ & O-allosyl-(1 $(\rightarrow 2)$-glc & $\mathrm{OH}$ & $\mathrm{OH}$ & $\mathrm{OH}$ & $\mathrm{H}$ \\
\hline Hypolaetin 7-O-[6"' -O-acetyl]- $\beta$-D-allosyl-( $(1 \rightarrow 2)-\beta-D$-glucoside (51) & $\mathrm{H}$ & $\mathrm{H}$ & 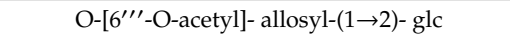 & $\mathrm{OH}$ & $\mathrm{OH}$ & $\mathrm{OH}$ & $\mathrm{H}$ \\
\hline Hypolaetin 7-O-[6"-O-acetyl]-allosyl-(1 $\rightarrow 2$ )glucoside (52) & $\mathrm{H}$ & $\mathrm{H}$ & O-[6"-O-acetyl]- allossyl-(1-2)-glc & $\mathrm{OH}$ & $\mathrm{OH}$ & $\mathrm{OH}$ & $\mathrm{H}$ \\
\hline 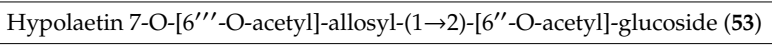 & $\mathrm{H}$ & $\mathrm{H}$ & 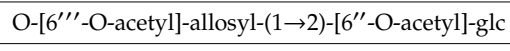 & $\mathrm{OH}$ & $\mathrm{OH}$ & $\mathrm{OH}$ & $\mathrm{H}$ \\
\hline Hypolaetin 7-O-[6"'-O-acetyl]-allosyl-( $(1 \rightarrow 2)$-[3"'-O-acetyl]-glucoside (54) & $\mathrm{H}$ & $\mathrm{H}$ & 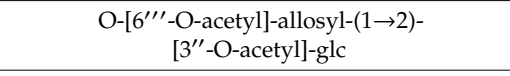 & $\mathrm{OH}$ & $\mathrm{OH}$ & $\mathrm{OH}$ & $\mathrm{H}$ \\
\hline 4'-Methyl-hypolaetin-7-O-allosyl-(1 $\rightarrow 2$ )-glucoside (55) & $\mathrm{H}$ & $\mathrm{H}$ & O-allosyl-(1 $(\rightarrow 2)$-glc & $\mathrm{OH}$ & $\mathrm{OH}$ & $\mathrm{OCH}_{3}$ & $\mathrm{H}$ \\
\hline $\begin{array}{c}4^{\prime} \text {-Methyl-hypolaetin-7-O-[6"' -O-acetyl]- } \beta \text {-D-allopyranosyl-( }(1 \rightarrow 2)-\beta \text { - } \\
\text { D-glucopyranoside (56) }\end{array}$ & $\mathrm{H}$ & $\mathrm{H}$ & O-[6"' & $\mathrm{OH}$ & $\mathrm{OH}$ & $\mathrm{OCH}_{3}$ & $\mathrm{H}$ \\
\hline $\begin{array}{l}4^{\prime} \text {-Methyl-hypolaetin-7-O-[6"-O-acetyl]- } \beta \text {-D-allopyranosyl-(1 } \rightarrow 2 \text { )- } \beta \text {-D- } \\
\text { glucopyranoside (57) }\end{array}$ & $\mathrm{H}$ & $\mathrm{H}$ & O-[6"-O-acetyl]-allosyl-(1 $\rightarrow 2)$ - glc & $\mathrm{OH}$ & $\mathrm{OH}$ & $\mathrm{OCH}_{3}$ & $\mathrm{H}$ \\
\hline $\begin{array}{l}\text { 4'-Methyl-hypolaetin-7-O-[6"'"-O-acetyl]-allosyl-(1 } \rightarrow 2) \text { - } \\
\text { [6"-O-acetyl]-glucoside (58) }\end{array}$ & $\mathrm{H}$ & $\mathrm{H}$ & 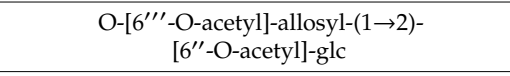 & $\mathrm{OH}$ & $\mathrm{OH}$ & $\mathrm{OCH}_{3}$ & $\mathrm{H}$ \\
\hline Selgin 7-O-glucoside (59) & $\mathrm{H}$ & $\mathrm{H}$ & O-glc & $\mathrm{H}$ & $\mathrm{OCH}_{3}$ & $\mathrm{OH}$ & $\mathrm{OH}$ \\
\hline Tricin 7-O-glucuronide (60) & $\mathrm{H}$ & $\mathrm{H}$ & O-glcA & $\mathrm{H}$ & $\mathrm{OCH}_{3}$ & $\mathrm{OH}$ & $\mathrm{OCH}$ \\
\hline Tricin 7-O-glucoside (61) & $\mathrm{H}$ & $\mathrm{H}$ & O-glc & $\mathrm{H}$ & $\mathrm{OCH}_{3}$ & $\mathrm{OH}$ & $\mathrm{OCH}$ \\
\hline Tricetin $3^{\prime}, 4^{\prime}, 5^{\prime}$-trimethyl-7-O-glucoside (62) & $\mathrm{H}$ & $\mathrm{H}$ & O-glc & $\mathrm{H}$ & $\mathrm{OCH}_{3}$ & $\mathrm{OCH}_{3}$ & $\mathrm{OCH}$ \\
\hline \multicolumn{8}{|c|}{$\mathrm{R}=\mathrm{O}$-glcA-glc $(2 \rightarrow 1)$} \\
\hline Palustrin (63) & $\mathrm{H}$ & $\mathrm{OH}$ & $\mathrm{OCH}_{3}$ & $\mathrm{H}$ & $\mathrm{H}$ & $\mathrm{H}$ & $\mathrm{H}$ \\
\hline \multicolumn{8}{|c|}{$\mathrm{R}=\mathrm{O}$-glcA } \\
\hline Palustrinoside (64) & $\mathrm{H}$ & $\mathrm{OH}$ & $\mathrm{OCH}_{3}$ & $\mathrm{H}$ & $\mathrm{H}$ & $\mathrm{H}$ & $\mathrm{H}$ \\
\hline
\end{tabular}

glc: glucose, glcA: glucuronide. 
Table 17. Chemical structures of poly-methylated flavonoids from Stachys spp.<smiles>[Y9]c1cc(-c2oc3c([NH])c(Br)c([R])c([Y])c3c(=O)c2[R3])cc([Y])c1[R3]</smiles>

\begin{tabular}{|c|c|c|c|c|c|c|c|}
\hline Name & $\mathbf{R}_{\mathbf{1}}$ & $\mathbf{R}_{\mathbf{2}}$ & $\mathbf{R}_{3}$ & $\mathbf{R}_{\mathbf{4}}$ & $\mathbf{R}_{\mathbf{5}}$ & $\mathbf{R}_{6}$ & $\mathbf{R}_{7}$ \\
\hline \multicolumn{8}{|c|}{$\mathrm{R}=\mathrm{OH}$} \\
\hline Velutin (luteolin 7,3'-dimethyl ether) (65) & $\mathrm{H}$ & $\mathrm{H}$ & $\mathrm{OCH}_{3}$ & $\mathrm{H}$ & $\mathrm{OCH}_{3}$ & $\mathrm{OH}$ & $\mathrm{H}$ \\
\hline Cirsimaritin (66) & $\mathrm{H}$ & $\mathrm{OCH}_{3}$ & $\mathrm{OCH}_{3}$ & $\mathrm{H}$ & $\mathrm{H}$ & $\mathrm{OH}$ & $\mathrm{H}$ \\
\hline 5,7,3'-Trihydroxy-6,4'-dimethoxyflavone (67) & $\mathrm{H}$ & $\mathrm{OCH}_{3}$ & $\mathrm{OH}$ & $\mathrm{H}$ & $\mathrm{OH}$ & $\mathrm{OCH}_{3}$ & $\mathrm{H}$ \\
\hline $5,7,3^{\prime}$-Trihydroxy- $6,8,4^{\prime}$-trimethoxyflavone (68) & $\mathrm{H}$ & $\mathrm{OCH}_{3}$ & $\mathrm{OH}$ & $\mathrm{OCH}_{3}$ & $\mathrm{OH}$ & $\mathrm{OCH}_{3}$ & $\mathrm{H}$ \\
\hline Xanthomicrol (69) & $\mathrm{H}$ & $\mathrm{OCH}_{3}$ & $\mathrm{OCH}_{3}$ & $\mathrm{OCH}_{3}$ & $\mathrm{H}$ & $\mathrm{OH}$ & $\mathrm{H}$ \\
\hline Sideritiflavone (70) & $\mathrm{H}$ & $\mathrm{OCH}_{3}$ & $\mathrm{OCH}_{3}$ & $\mathrm{OCH}_{3}$ & $\mathrm{OH}$ & $\mathrm{OH}$ & $\mathrm{H}$ \\
\hline 8-Methoxycirsilineol (71) & $\mathrm{H}$ & $\mathrm{OCH}_{3}$ & $\mathrm{OCH}_{3}$ & $\mathrm{OCH}_{3}$ & $\mathrm{OCH}_{3}$ & $\mathrm{OH}$ & $\mathrm{H}$ \\
\hline Eupatorin (72) & $\mathrm{H}$ & $\mathrm{OCH}_{3}$ & $\mathrm{OCH}_{3}$ & $\mathrm{H}$ & $\mathrm{OH}$ & $\mathrm{OCH}_{3}$ & $\mathrm{H}$ \\
\hline Eupatilin (72a) & $\mathrm{H}$ & $\mathrm{OCH}_{3}$ & $\mathrm{OH}$ & $\mathrm{H}$ & $\mathrm{OCH}_{3}$ & $\mathrm{OCH}_{3}$ & $\mathrm{H}$ \\
\hline Eupatilin-7-methyl ether (73) & $\mathrm{H}$ & $\mathrm{OCH}_{3}$ & $\mathrm{OCH}_{3}$ & $\mathrm{H}$ & $\mathrm{OCH}_{3}$ & $\mathrm{OCH}_{3}$ & $\mathrm{H}$ \\
\hline Salvigenin (74) & $\mathrm{H}$ & $\mathrm{OCH}_{3}$ & $\mathrm{OCH}_{3}$ & $\mathrm{H}$ & $\mathrm{H}$ & $\mathrm{OCH}_{3}$ & $\mathrm{H}$ \\
\hline 5-Hydroxy-6,7,8,3', $4^{\prime}$-pentamethoxyflavone (75) & $\mathrm{H}$ & $\mathrm{OCH}_{3}$ & $\mathrm{OCH}_{3}$ & $\mathrm{OCH}_{3}$ & $\mathrm{OCH}_{3}$ & $\mathrm{OCH}_{3}$ & $\mathrm{H}$ \\
\hline 5, 4'-Dihydroxy - 6,7,8,3'-tetramethoxyflavone (76) & $\mathrm{H}$ & $\mathrm{OCH}_{3}$ & $\mathrm{OCH}_{3}$ & $\mathrm{OCH}_{3}$ & $\mathrm{OCH}_{3}$ & $\mathrm{OH}$ & $\mathrm{H}$ \\
\hline 5, 4' -Dihydroxy-7, $3^{\prime}, 5^{\prime}$-trimethoxyflavone (77) & $\mathrm{H}$ & $\mathrm{H}$ & $\mathrm{OCH}_{3}$ & $\mathrm{H}$ & $\mathrm{OCH}_{3}$ & $\mathrm{OH}$ & $\mathrm{OCH}_{3}$ \\
\hline Viscosine (5,7, $4^{\prime}$-trihydroxy-3,6-dimethoxyflavone) (78) & $\mathrm{OCH}_{3}$ & $\mathrm{OCH}_{3}$ & $\mathrm{OH}$ & $\mathrm{H}$ & $\mathrm{H}$ & $\mathrm{OH}$ & $\mathrm{H}$ \\
\hline Kumatakenin (kaempferol 3,7-dimethyl ether) (79) & $\mathrm{OCH}_{3}$ & $\mathrm{H}$ & $\mathrm{OCH}_{3}$ & $\mathrm{H}$ & $\mathrm{H}$ & $\mathrm{OH}$ & $\mathrm{H}$ \\
\hline Pachypodol (quercetin 3,7,3'-trimethyl ether) (80) & $\mathrm{OCH}_{3}$ & $\mathrm{H}$ & $\mathrm{OCH}_{3}$ & $\mathrm{H}$ & $\mathrm{OCH}_{3}$ & $\mathrm{OH}$ & $\mathrm{H}$ \\
\hline Penduletin (81) & $\mathrm{OCH}_{3}$ & $\mathrm{OCH}_{3}$ & $\mathrm{OCH}_{3}$ & $\mathrm{H}$ & $\mathrm{H}$ & $\mathrm{OH}$ & $\mathrm{H}$ \\
\hline $5,3^{\prime}, 4^{\prime}$-Trihydroxy-3,6,7,8-tetramethoxyflavone (82) & $\mathrm{OCH}_{3}$ & $\mathrm{OCH}_{3}$ & $\mathrm{OCH}_{3}$ & $\mathrm{OCH}_{3}$ & $\mathrm{OH}$ & $\mathrm{OH}$ & $\mathrm{H}$ \\
\hline Calycopterin (83) & $\mathrm{OCH}_{3}$ & $\mathrm{OCH}_{3}$ & $\mathrm{OCH}_{3}$ & $\mathrm{OCH}_{3}$ & $\mathrm{H}$ & $\mathrm{OH}$ & $\mathrm{H}$ \\
\hline Chrysosplenetin (84) & $\mathrm{OCH}_{3}$ & $\mathrm{OCH}_{3}$ & $\mathrm{OCH}_{3}$ & $\mathrm{H}$ & $\mathrm{OCH}_{3}$ & $\mathrm{OH}$ & $\mathrm{H}$ \\
\hline 5-Hydroxy-3,6,7,4'-tetramethoxyflavone (85) & $\mathrm{OCH}_{3}$ & $\mathrm{OCH}_{3}$ & $\mathrm{OCH}_{3}$ & $\mathrm{H}$ & $\mathrm{H}$ & $\mathrm{OCH}_{3}$ & $\mathrm{H}$ \\
\hline 5,8-Dihydroxy-3,6,7,4'-tetramethoxyflavone (86) & $\mathrm{OCH}_{3}$ & $\mathrm{OCH}_{3}$ & $\mathrm{OCH}_{3}$ & $\mathrm{OH}$ & $\mathrm{H}$ & $\mathrm{OCH}_{3}$ & $\mathrm{H}$ \\
\hline Casticin (87) & $\mathrm{OCH}_{3}$ & $\mathrm{OCH}_{3}$ & $\mathrm{OCH}_{3}$ & $\mathrm{H}$ & $\mathrm{OH}$ & $\mathrm{OCH}_{3}$ & $\mathrm{H}$ \\
\hline $\begin{array}{c}\text { 5-Hydroxy-3,6,7,8,4' - pentamethoxyflavone } \\
\text { (5-hydroxyauranetin) (88) }\end{array}$ & $\mathrm{OCH}_{3}$ & $\mathrm{OCH}_{3}$ & $\mathrm{OCH}_{3}$ & $\mathrm{OCH}_{3}$ & $\mathrm{H}$ & $\mathrm{OCH}_{3}$ & $\mathrm{H}$ \\
\hline $5,4^{\prime}$-Dihydroxy $-3,6,7,8,3^{\prime}$ - pentamethoxyflavone (89) & $\mathrm{OCH}_{3}$ & $\mathrm{OCH}_{3}$ & $\mathrm{OCH}_{3}$ & $\mathrm{OCH}_{3}$ & $\mathrm{OCH}_{3}$ & $\mathrm{OH}$ & $\mathrm{H}$ \\
\hline \multicolumn{8}{|c|}{$\mathrm{R}=\mathrm{OCH}_{3}$} \\
\hline 4'-Hydroxy- 3,5,7,3'-tetramethoxyflavone (90) & $\mathrm{OCH}_{3}$ & $\mathrm{H}$ & $\mathrm{OCH}_{3}$ & $\mathrm{H}$ & $\mathrm{OCH}_{3}$ & $\mathrm{OH}$ & $\mathrm{H}$ \\
\hline
\end{tabular}

Table 18. Chemical structures of flavonols from Stachys spp.<smiles>[Y9]c1ccc(-c2oc3cc([14CH3])cc(O)c3c(=O)c2Br)cc1[135I]</smiles>

\begin{tabular}{ccccc}
\hline Name & $\mathbf{R}_{\mathbf{1}}$ & $\mathbf{R}_{\mathbf{2}}$ & $\mathbf{R}_{\mathbf{3}}$ & $\mathbf{R}_{\mathbf{4}}$ \\
\hline Kaempferol (91) & $\mathrm{OH}$ & $\mathrm{OH}$ & $\mathrm{H}$ & $\mathrm{OH}$ \\
Isorhamnetin (92) & $\mathrm{OH}$ & $\mathrm{OH}$ & $\mathrm{OCH}_{3}$ & $\mathrm{OH}$ \\
Quercetin 3-O-rutinoside (93) & O-rut & $\mathrm{OH}$ & $\mathrm{OH}$ & $\mathrm{OH}$ \\
Isorhamnetin 3-O-rutinoside (94) & O-rut & $\mathrm{OH}$ & $\mathrm{OCH}_{3}$ & $\mathrm{OH}$ \\
\hline
\end{tabular}

rut: rutinoside. 
Table 19. Chemical structures of flavanones from Stachys spp.<smiles>[R3]c1cc(O)c2c(c1)OC(c1ccc([123I])c([Y])c1)CC2=O</smiles>

\begin{tabular}{cccc}
\hline Name & $\mathbf{R}$ & $\mathbf{R}_{\mathbf{1}}$ & $\mathbf{R}_{\mathbf{2}}$ \\
\hline Eriodictyol (95) & $\mathrm{OH}$ & $\mathrm{OH}$ & $\mathrm{OH}$ \\
Naringenin (96) & $\mathrm{H}$ & $\mathrm{OH}$ & $\mathrm{OH}$ \\
Hesperidin (97) & $\mathrm{OH}$ & $\mathrm{OCH}_{3}$ & O-rut \\
\hline \multicolumn{3}{c}{ rut: rutinoside. }
\end{tabular}

Table 20. Chemical structure of biflavonoid from Stachys spp.

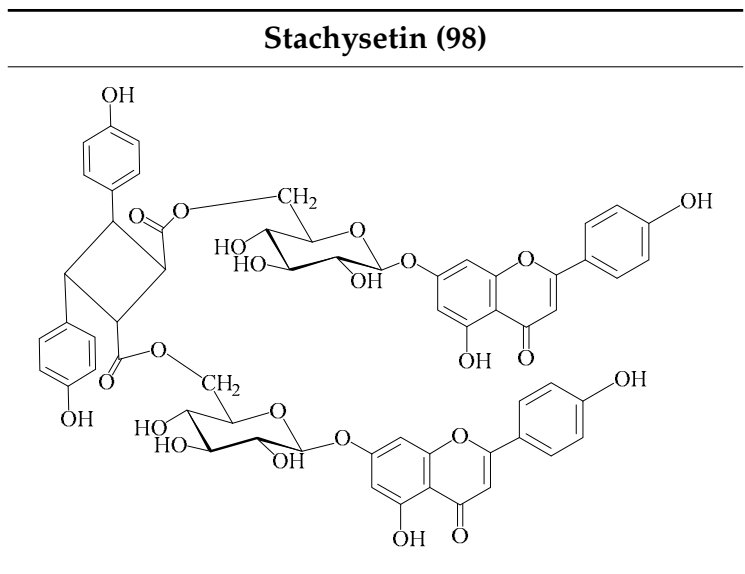

\subsection{Phenolic Derivatives; Acetophenone Derivatives}

Regarding the phenolic derivatives of genus Stachys (Tables 7 and 21), mainly chlorogenic acid (103) was appeared in nine Stachys species; S. candida [78], S. iva [56], S. cretica (S. cretica subsp. smyrnaea [81], S. cretica subsp. mersinaea [108], S. cretica subsp. vacillans [112]), S. lanata [82], S. tmolea [85], S. thirkei [84], S. recta [14], S. palustris [104] and S. officinalis [111]. The isomers of chlorogenic acid $(\mathbf{1 0 2}, \mathbf{1 0 4}, \mathbf{1 0 5})$ also reported in S. atherocalyx [110], S. recta [14] and S. palustris [23,104]. Caffeic (108) and p-coumaric (106) acids were found in two Stachys spp. [104,110]. Moreover, Kirkan (2019) identified vanillic (100) and syringic (101) acids from the aerial parts of S. cretica subsp. vacillans [112]. Though, 4-hydroxybenzoic acid (99) was reported from S. tmolea [85]. Arbutin (107) was also identified in the aerial parts of S. germanica subsp. salviifolia [109]. One study also reported the presence of acetophenone derivatives from the roots of $S$. lanata, namely androsin (109), neolloydosin (110) and glucoacetosyringone (111) (Tables 8 and 22) [82]. The isolation of the latter compounds might be attributed to the different investigated plant parts (roots). 
Table 21. Chemical structures of phenolic derivatives from Stachys spp.

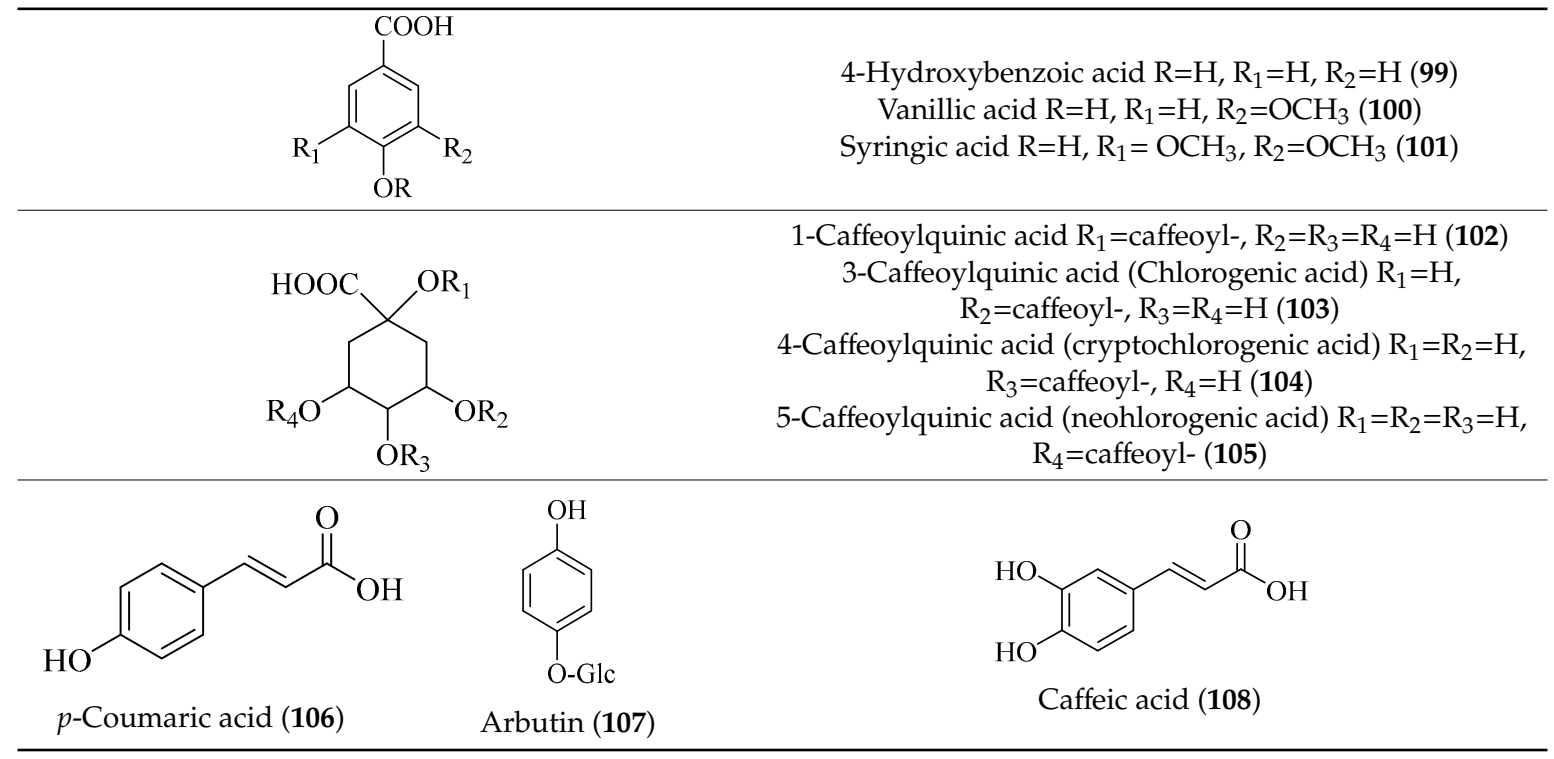

Glc: glucose.

Table 22. Chemical structures of acetophenone glycosides from Stachys spp.

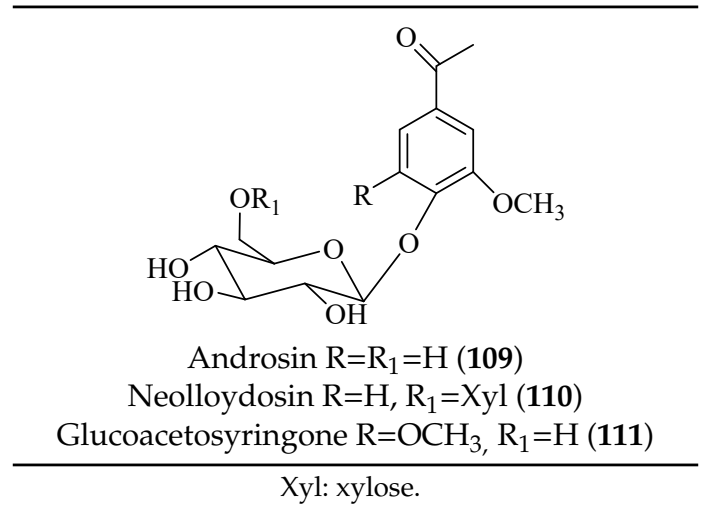

\subsection{Lignans}

Lignans are types of polyphenols with diverse structures. Although these bioactive compounds were presented in Lamiaceae family [149], a few studies reported their existence in plants of genus Stachys. Specifically, three lignans categorizing into two furanofuran-type derivatives (sesamin and paulownin) and one benzofuran-type lignan (urolignoside) were reported in two species of the subgenus Stachys (Tables 9 and 23). Laggoune et al. (2016) isolated sesamin (112) and paulownin (113) from the aerial parts of $S$. mialhesii [103], while urolignoside (114) was isolated from the aerial parts of S. tetragona [100]. Given that up to now there is no study reported the presence of lignans in the subgenus Betonica, the identification of lignans might be considered as a chemotaxonomic difference between the two subgenera Stachys and Betonica. 
Table 23. Chemical structures of lignans from Stachys spp.

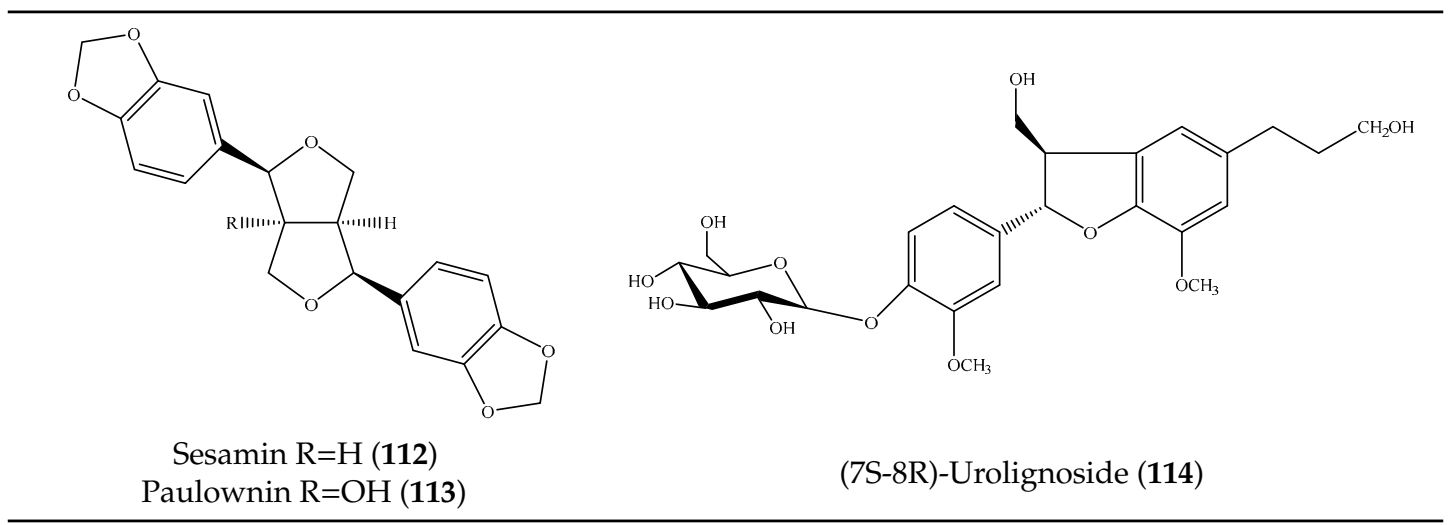

\subsection{Phenylethanoid Glycosides; Phenylpropanoid Glucosides}

The present review unveiled 29 phenylethanoid glycosides in 17 Stachys species (Tables 10 and 24). Acteoside or verbascoside (118) was the most abundant found in 16 Stachys spp. of all sections through this survey. Additional phenylethanoid glycosides isolated and identified from this genus includes martynoside, leucosceptoside A and lavandulifoliosides. Lavandulifolioside A (or stachysoside A) (129) was firstly isolated from the methanol extract of the aerial parts of S. lavandulifolia in 1988 [115], while in 2011 Delazar et al. (2011) isolated lavandulifolioside B (130) from the same plant, for the first time [12]. Moreover, three phenylethanoid glycosides were reported from the aerial parts of S. byzantina (section Eriostomum), including verbascoside (118), 2'-O-arabinosyl verbascoside (122) and aeschynanthoside C (133) [35]. Among them, the first and the last compound has been isolated only from the specific species. A survey conducted by Murata and co-workers (2008) reported ten phenylethanoid glycosides from different plant parts [82]. In the aforementioned study, leonoside B (or stachysoside D) (134) and martynoside (135) were mentioned from the aerial parts of S. lanata, while from the roots of the specific species were reported eight phenylethanoid glycosides, namely rhodioloside (115), verbasoside (116), 2-phenylethyl-D-xylopyranosyl-(1 $\rightarrow 6)$-D-glucopyranoside (117), verbascoside (118), isoacteoside (119), darendoside B (120), campneoside II (121) and campneoside I (136). It is remarkable to point out that compounds 115, 117 and 120 haven't been reported in other Stachys species. This might be attributed to the fact that the plant material was roots. Another study carried out by Karioti et al. (2010) focused on the phenolic compounds from the aerial parts of S. recta, and reported many phenylethanoid glycosides from its aerial parts, including acteoside (118), isoacteoside (119), $\beta-\mathrm{OH}$-acteoside (121), betunyoside $\mathrm{E}$ (127), campneoside I (136), forsythoside $\mathrm{B}$ (137), $\beta$-OH-forsythoside B methyl ether (138) [14]. Furthermore, lamiophloside A (141) was isolated with some other phenylethanoid glycosides from the aerial parts of $S$. tetragona [100]. Of great interest is that our survey revealed that this constituent is mentioned only in the specific species. Two rare phenylethanoid glycosides, parviflorosides A-B (142-143) were isolated from the whole plant of S. parviflora [120]. These two compounds are characterised by the presence of a third saccharide (rhamnose) linked to the proton $\mathrm{H}-2^{\prime}$ of glucose, comparing to others common phenylethanoid glycosides where the connection of the third saccharide is in proton $\mathrm{H}-3^{\prime}$ of glucose. Of great interest is that S. parviflora is now considered as the monotypic genus Phlomidoschema (only P. parviflorum (Benth.) Vved.) [2]. Furthermore, leonoside A (or stachysoside B) (139) was isolated with other three phenylethanoid glucosides from the whole plant of S. riederi [114]. To be mentioned that phenylethanoid glycosides were reported in both subgenera of genus Stachys.

Apart from phenylethanoid glucosides, Murata et al. (2008) mentioned two phenylpropanoid glucosides in the roots of S. lanata (subg. Stachys; sect. Eriostomum), coniferin (144) and syringin (145) (Tables 11 and 25) [82]. It is worth to mention that the isolation of phenylpropanoid glucosides only from the specific plant, might be assigned to the different studied plant material (roots). 
Table 24. Chemical structures of phenylethanoid glycosides from Stachys spp.

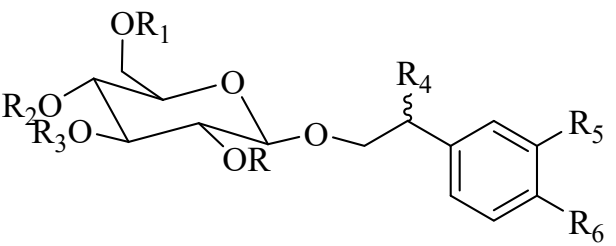

\begin{tabular}{|c|c|c|c|c|c|c|c|}
\hline \multicolumn{3}{|c|}{ Caffeic acid } & \multicolumn{2}{|c|}{$\mathrm{HO}^{-}$} & \multicolumn{3}{|c|}{ Ferulic acid } \\
\hline Name & $\mathbf{R}_{\mathbf{1}}$ & $\mathbf{R}_{\mathbf{2}}$ & $\mathbf{R}_{3}$ & $\mathbf{R}_{4}$ & $\mathbf{R}_{5}$ & $\mathbf{R}_{6}$ & $\mathbf{R}$ \\
\hline Rhodioloside (Salidroside) (115) & $\mathrm{H}$ & $\mathrm{H}$ & $\mathrm{H}$ & $\mathrm{H}$ & $\mathrm{H}$ & $\mathrm{OH}$ & $\mathrm{H}$ \\
\hline Verbasoside (decaffeoyl-acteoside) (116) & $\mathrm{H}$ & $\mathrm{H}$ & Rha & $\mathrm{H}$ & $\mathrm{OH}$ & $\mathrm{OH}$ & $\mathrm{H}$ \\
\hline 2-Phenylethyl-D-xylopyranosyl-(1 $\rightarrow 6)$-D-glucopyranoside (117) & Xyl & $\mathrm{H}$ & $\mathrm{H}$ & $\mathrm{H}$ & $\mathrm{H}$ & $\mathrm{H}$ & $\mathrm{H}$ \\
\hline Acteoside (Verbascoside) (118) & $\mathrm{H}$ & Caf & Rha & $\mathrm{H}$ & $\mathrm{OH}$ & $\mathrm{OH}$ & $\mathrm{H}$ \\
\hline Isoacteoside (119) & Caf & $\mathrm{H}$ & Rha & $\mathrm{H}$ & $\mathrm{OH}$ & $\mathrm{OH}$ & $\mathrm{H}$ \\
\hline Darendoside B (deacyl-martynoside) (120) & $\mathrm{H}$ & $\mathrm{H}$ & Rha & $\mathrm{H}$ & $\mathrm{OH}$ & $\mathrm{OCH}_{3}$ & $\mathrm{H}$ \\
\hline$\beta$-OH-Acteoside (Campneoside II) (121) & $\mathrm{H}$ & Caf & Rha & $\mathrm{OH}$ & $\mathrm{OH}$ & $\mathrm{OH}$ & $\mathrm{H}$ \\
\hline 2'-O-Arabinosyl verbascoside (122) & $\mathrm{H}$ & Caf & Rha & $\mathrm{H}$ & $\mathrm{OH}$ & $\mathrm{OH}$ & Ara \\
\hline Betonyoside A (123) & $\mathrm{H}$ & Fer & Rha & $\mathrm{OH}$ & $\mathrm{OH}$ & $\mathrm{OH}$ & $\mathrm{H}$ \\
\hline Betonyoside B/C (isomers) (124/125) & Fer & $\mathrm{H}$ & Rha & $\mathrm{OH}$ & $\mathrm{OH}$ & $\mathrm{OH}$ & $\mathrm{H}$ \\
\hline Betonyoside D (126) & Api & Cis-fer & Rha & $\mathrm{H}$ & $\mathrm{OH}$ & $\mathrm{OCH}_{3}$ & $\mathrm{H}$ \\
\hline Betonyoside E (127) & Api & Fer & Rha & $\mathrm{OH}$ & $\mathrm{OH}$ & $\mathrm{OH}$ & $\mathrm{H}$ \\
\hline Betonyoside F (128) & $\mathrm{H}$ & Caf & Rha-Api & $\mathrm{H}$ & $\mathrm{OH}$ & $\mathrm{OH}$ & $\mathrm{H}$ \\
\hline Lavandulifolioside A (Stachysoside A) (129) & $\mathrm{H}$ & Caf & Rha-Ara & $\mathrm{H}$ & $\mathrm{OH}$ & $\mathrm{OH}$ & $\mathrm{H}$ \\
\hline Lavandulifolioside B (130) & $\mathrm{H}$ & $4^{\prime}$-methyl-Fer & Rha-Ara & $\mathrm{H}$ & $\mathrm{OCH}_{3}$ & $\mathrm{OH}$ & $\mathrm{H}$ \\
\hline Leucosceptoside A (131) & $\mathrm{H}$ & Fer & Rha & $\mathrm{H}$ & $\mathrm{OH}$ & $\mathrm{OH}$ & $\mathrm{H}$ \\
\hline Leucosceptoside B (132) & Api & Fer & Rha & $\mathrm{H}$ & $\mathrm{OH}$ & $\mathrm{OCH}_{3}$ & $\mathrm{H}$ \\
\hline Aeschynanthoside C (133) & $\mathrm{H}$ & Fer & Xyl & $\mathrm{H}$ & $\mathrm{OH}$ & $\mathrm{OCH}_{3}$ & $\mathrm{H}$ \\
\hline Leonoside B (Stachysoside D) (134) & $\mathrm{H}$ & Fer & Rha-Ara & $\mathrm{H}$ & $\mathrm{OH}$ & $\mathrm{OCH}_{3}$ & $\mathrm{H}$ \\
\hline Martynoside (135) & $\mathrm{H}$ & Fer & Rha & $\mathrm{H}$ & $\mathrm{OH}$ & $\mathrm{OCH}_{3}$ & $\mathrm{H}$ \\
\hline Campneoside I (136) & $\mathrm{H}$ & Caf & Rha & $\mathrm{OCH}_{3}$ & $\mathrm{OH}$ & $\mathrm{OH}$ & $\mathrm{H}$ \\
\hline
\end{tabular}


Table 24. Cont.

\begin{tabular}{|c|c|c|c|c|c|c|c|}
\hline Name & $\mathbf{R}_{1}$ & $\mathbf{R}_{2}$ & $\mathbf{R}_{3}$ & $\mathbf{R}_{4}$ & $\mathbf{R}_{5}$ & $\mathbf{R}_{6}$ & $\mathbf{R}$ \\
\hline Forsythoside B (137) & Api & Caf & Rha & $\mathrm{H}$ & $\mathrm{OH}$ & $\mathrm{OH}$ & $\mathrm{H}$ \\
\hline$\beta$-OH-Forsythoside B methyl ether (138) & Api & Caf & Rha & $\mathrm{OCH}_{3}$ & $\mathrm{OH}$ & $\mathrm{OH}$ & $\mathrm{H}$ \\
\hline Leonoside A (Stachysoside B) (139) & $\mathrm{H}$ & Fer & Rha-Ara & $\mathrm{H}$ & $\mathrm{OH}$ & $\mathrm{OH}$ & $\mathrm{H}$ \\
\hline * Stachysoside C (140) & $\mathrm{H}$ & Fer & Rha-Ara & $\mathrm{H}$ & $\mathrm{OH}$ & $\mathrm{OH}$ & $\mathrm{H}$ \\
\hline Lamiophloside A (141) & Api & Fer & Rha & $\mathrm{H}$ & $\mathrm{OCH}_{3}$ & $\mathrm{OH}$ & $\mathrm{H}$ \\
\hline Parvifloroside A (142) & $\stackrel{\mathrm{H}}{\mathrm{H}}$ & Caf & $\mathrm{H}$ & $\mathrm{H}$ & $\mathrm{OH}$ & $\mathrm{OH}$ & Rha \\
\hline Parvifloroside B (143) & Caf & $\mathrm{H}$ & $\mathrm{H}$ & $\mathrm{H}$ & $\mathrm{OH}$ & $\mathrm{OH}$ & Rha \\
\hline
\end{tabular}

Caf: Caffeic acid, Fer: Ferulic acid, Api: Apioside, Rha: Rhamnoside, Ara: Arabinoside, Xyl: Xyloside, ${ }^{*}$ might be synonym of Leonoside B.

Table 25. Chemical structures of phenylpropanoid glucosides from Stachys spp.

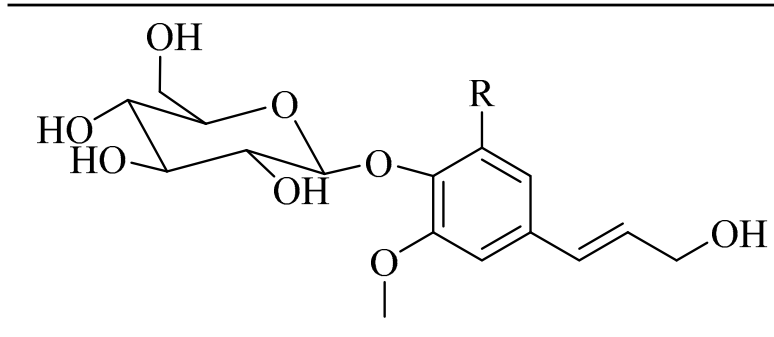

Coniferin $\mathrm{R}=\mathrm{H}(\mathbf{1 4 4}) \quad$ Syringin $\mathrm{R}=\mathrm{OCH}_{3}(\mathbf{1 4 5})$ 


\subsection{Iridoids}

Iridoids are among the major chemical compounds found in genus Stachys. According to Tundis et al. (2014), iridoids are considered as good chemotaxonomic markers of this genus [3]. Accumulating phytochemical studies have reported diverse types of iridoids [3]. The present review summarises all these studies, exemplifying 38 Stachys species which their iridoid cargo has been investigated (Tables 12 and 26). Harpagide (148; 31 species) and its acetyl derivative; 8 acetyl-harpagide (150; 28 species) are of common occurrence in genus Stachys and might be considered as characteristic iridoids of these plants. Furthermore, ajugol (146; 18 species), ajugoside (147; 18 species), melittoside (166; 17 species), monomelittoside (165; 4 species) and 5-allosyloxy-aucubin or 5-O-allopyranosyl-monomelittoside (167; 4 species/1 subsp.) were also mentioned in various species. Allobetonicoside (161) was firstly isolated from the aerial parts of S. officinalis [127] and then from the aerial parts of S. glutinosa [122] and of S. macrantha [117]. The latter study also mentioned the isolation of cinnamoyl-harpagide derivative, macranthoside (156), for the first time. To be mentioned that Jeker et al. (1989) also isolated 6-O-acetylmioporoside (155) from the aerial parts of S. officinalis [127]. In addition, two species revealed the presence of 8-epi-loganic acid (157), 8-epi-loganin (159) and gardoside (160) [20,56], as well as 7-O-acetyl-8-epi-loganic acid (158) was only mentioned from the aerial parts of S. spinosa [98]. Of note, Iannuzzi et al. (2019) isolated from the leaves of S. ocymastrum (syn. S. hirta L.) five iridoids which haven't been documented in other species, namely $6 \beta$-acetoxyipolamiide (172) 6ß-hydroxyipolamiide (173), ipolamiide (174), ipolamiidoside (175) and lamiide (176) [123]. A study conducted by Háznagy-Radnai (2006) examined the phytochemical profiles of Stachys spp. growing in Hungary, reporting the iridoid content of ten taxa [124]. Murata and co-workers (2008) isolated five new esters of monomelittoside from the aerial parts and roots of S. lanata [82]. In particular, stachysosides E (168), G-H (170-171) were found in roots, while stachysosides E (168) and F (169) were discovered from the aerial parts of the specific species. It is important to be mentioned the detection of a new iridoid diglycoside, $4^{\prime}$-O- $\beta$-D-galactopyranosyl-teuhircoside (162), which was isolated from the flowering aerial parts of S. alopecuros subsp. divulsa [119]. Muñoz et al. (2001) reported the presence of 5-desoxy-harpagide (151) and 5-desoxy-8-acetyl-harpagide (152) from the aerial parts of S. grandidentata [129]. Notably, this review unveiled some differences in iridoids among subgenera Stachys and Betonica. Firstly, it was observed that there is no report for the presence of monomelittoside or melittoside derivatives in the subgenus Betonica. Secondly, reptoside (153) was found in two species of subgenus Betonica (S. macrantha and S. officinalis) and not in the plants of subgenus Stachys. 
Table 26. Chemical structures of iridoids from Stachys spp.

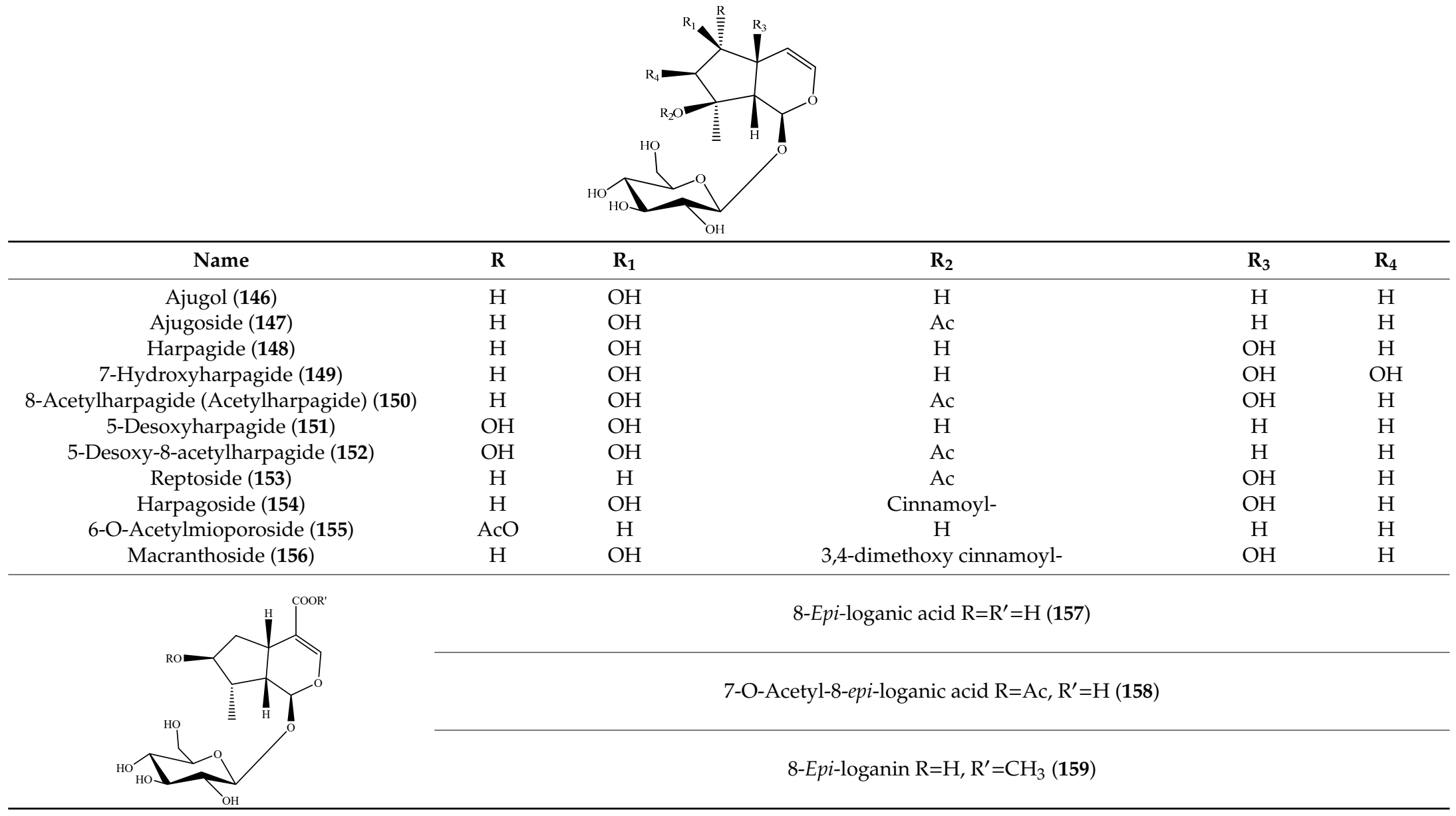


Table 26. Cont.

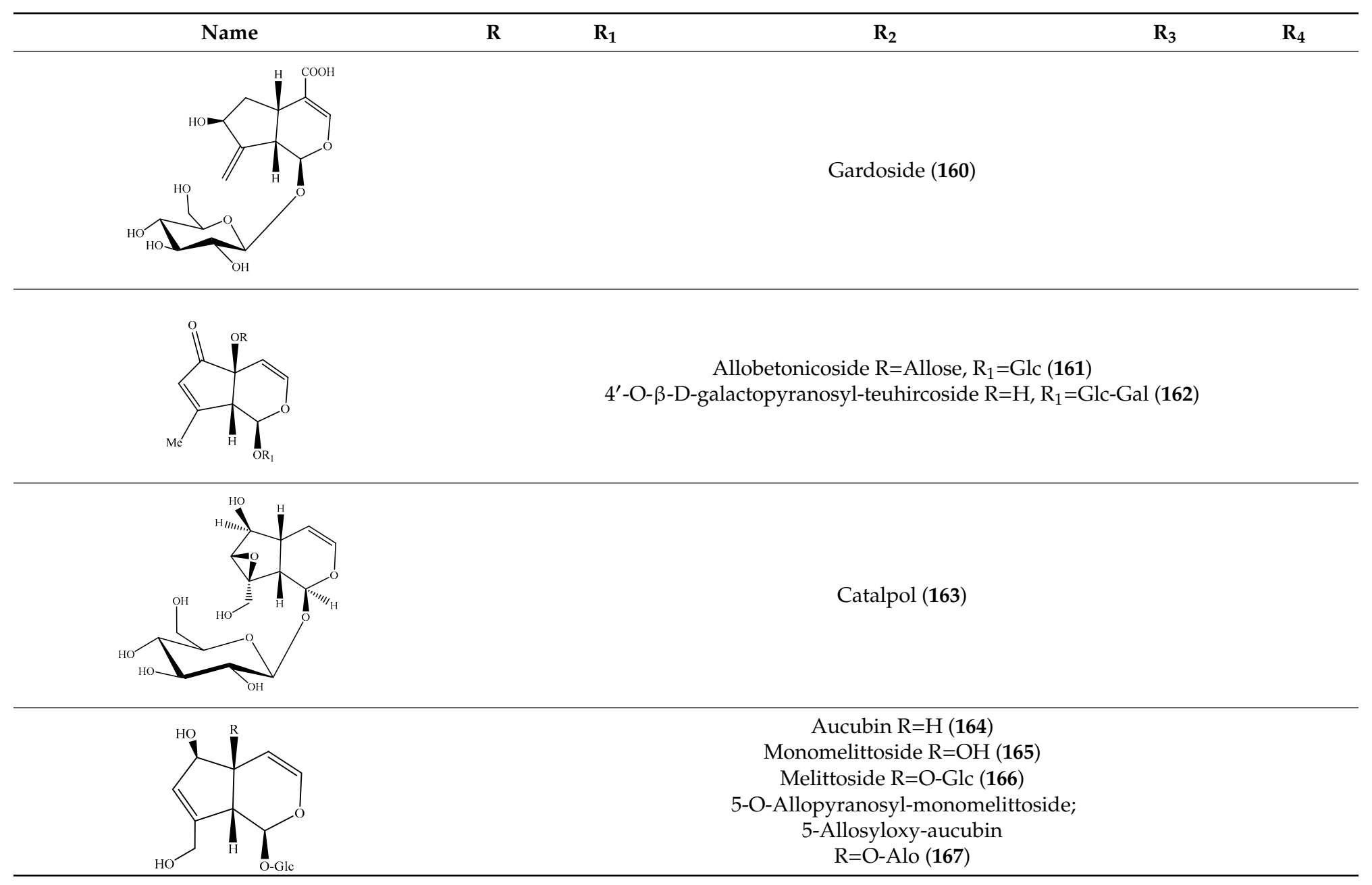


Table 26. Cont.

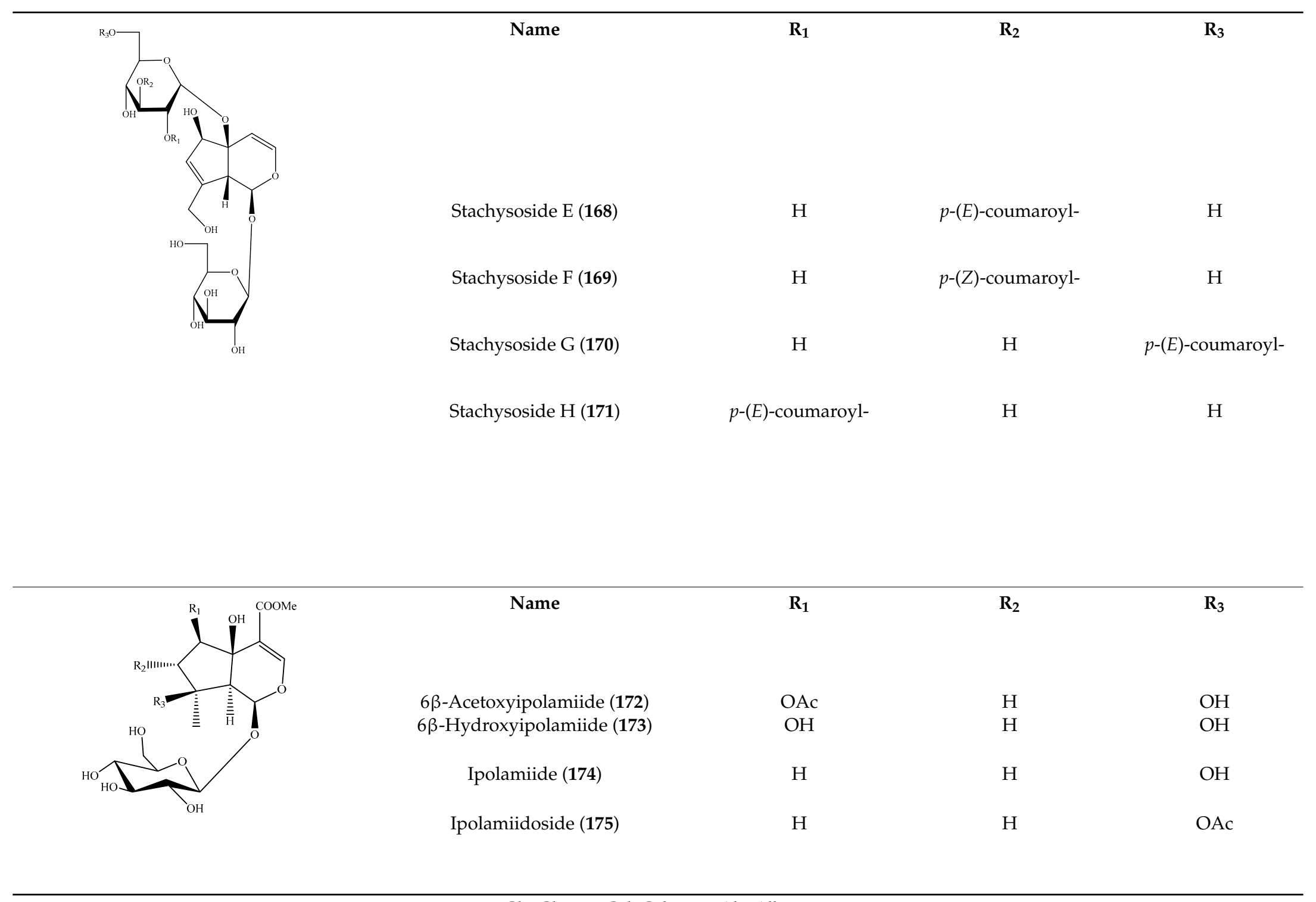




\subsection{Diterpenes}

A landmark study for diterpenes of genus Stachys is the review article of Piozzi and Bruno (2011), including all the reported diterpenoids from roots and aerial parts of Stachys spp [21]. Up to now, several types of diterpenes have been mentioned, comprising types of neo-clerodane, labdane, rosane and ent-kaurene skeleton (Tables 13 and 27). The most common type is the neo-clerodane skeleton derivates, as they were found in various species. S. aegyptiaca has thoroughly studied for its phytochemical profile. A study conducted by Hegazy et al. (2017) reported the discovery of three new neo-clerodane diterpenoids from the aerial parts of the aforementioned plant, namely stachaegyptins A-C (190-192) [106]. One year later, two new compounds were mentioned; stachaegyptins D-E (193-194) [131], while in a current work stachaegyptins F-H were isolated (195-197) [133]. Moreover, stachysperoxide (189) was isolated from the S. aegyptiaca [132]. These stachaegyptin derivatives and stachysperoxide reported only in the species S. aegyptiaca and might be a characteristic chemical compound of the specific plant of the section Ambleia. Derkach (1998) mentioned the compounds annuanone (cis-clerodane type) (181), stachylone (182) and stachone (183) in five species of the subgenus Stachys; S. atherocalyx, S. inflata, S. iberica and S. sylvatica [134]. Other neo-clerodane type diterpenes which were found in many species are roseostachenone (184), roseostachone (185), roseostachenol (186) and roseotetrol (187). Ruiu and co-workers (2015) explored the aerial parts of $S$. glutinosa, isolating roseostachenone and the new neo-clerodane diterpene, $3 \alpha, 4 \alpha$-epoxyroseostachenol (188) [107]. Furthermore, labdane type derivatives were occurred in the genus Stachys. Fazio et al. (1994) investigated the aerial parts of S. mucronata and isolated three labdane skeleton compounds; ribenone (198), ribenol (199) and 13-epi-sclareol (200) [57]. The latter compound has also been found in S. rosea [141]. Paternostro et al. (2000) studied the aerial parts of S. plumosa, determining the following labdane type derivatives (+)-6-deoxyandalusol (201), 13-epi-jabugodiol (202) and (+)-plumosol (203) [144]. The compound (+)-6-deoxyandalusol were also found in S. distans and S. ionica [139]. Some ent-kaurene derivatives were reported in S. aegyptiaca [130], S. lanata [135] and S. sylvatica [142]. Moreover, one abietane diterpenoid, horminone (211), was isolated from the aerial parts of S. mialhesii [103]. It is noteworthy to be underlay the presence of two rare rosane type diterpenes in the aerial parts of S. parviflora, namely stachyrosanes 1 (212) and 2 (213) [134]. In addition, six diterpene lactone derivatives, i.e., betolide (214), betonicolide (215) and betonicosides A-D (216-219) were found in the species S. officinalis [143,145] and S. scardica [143] of the subgenus Betonica.

In the context of chemotaxonomic significance, it could be observed that species of subgenus Stachys product mainly neo-clerodane and labdane type derivatives, while the plants of subgenus Betonica biosynthesized diterpene lactone derivatives. Thus, the latter derivatives might be recognised as characteristic chemotaxonomic markers of subgenus Betonica. Another important chemotaxonomic point is reported by Piozzi et al. (2002), mentioning that (+)-6-deoxyandalusol has been determined only in three Stachys species of eastern part of the Mediterranean region [139]. 
Table 27. Diterpenes from Stachys spp.

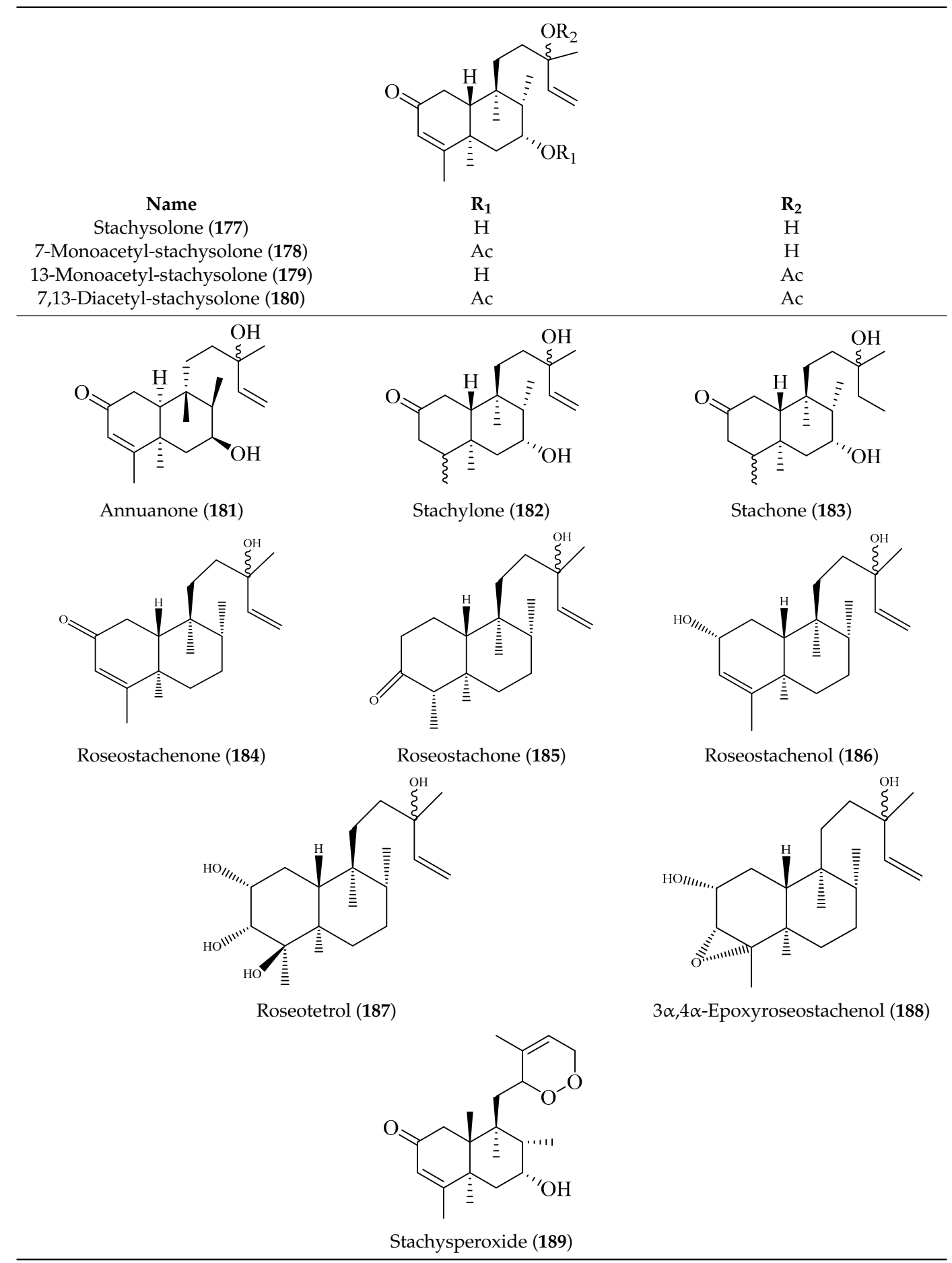


Table 27. Cont.

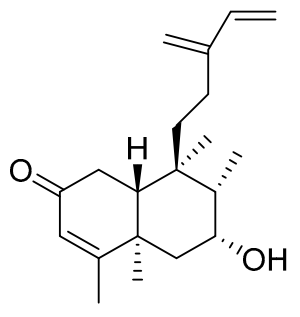

Stachaegyptin A (190)

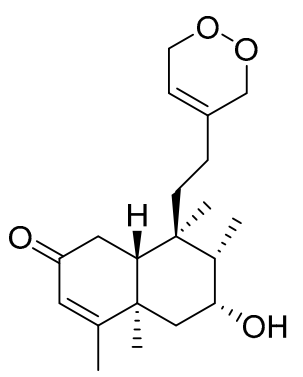

Stachaegyptin C (192)

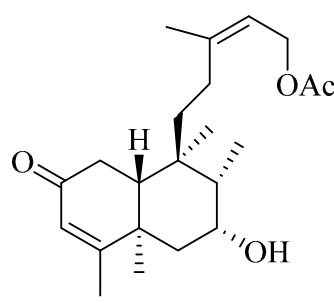

Stachaegyptin E (194)

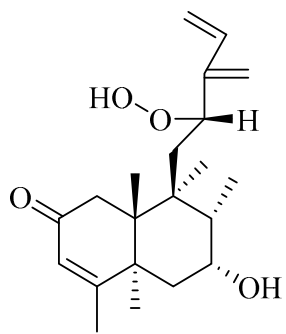

Stachaegyptin G (196)

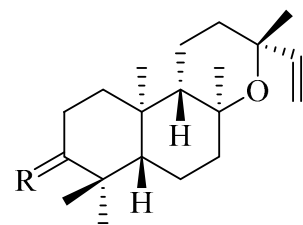

Ribenone $\mathrm{R}=\mathrm{O}$ (198)

Ribenol $\mathrm{R}=\alpha \mathrm{OH}, \beta \mathrm{H}(\mathbf{1 9 9})$

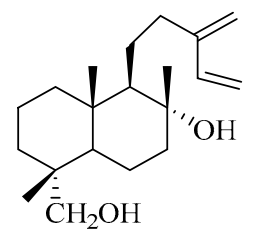

(+)-6-Deoxyandalusol (201)

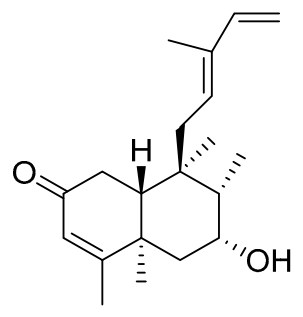

Stachaegyptin B (191)

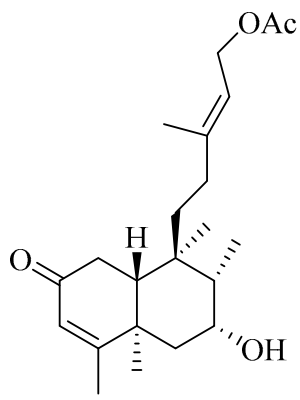

Stachaegyptin D (193)

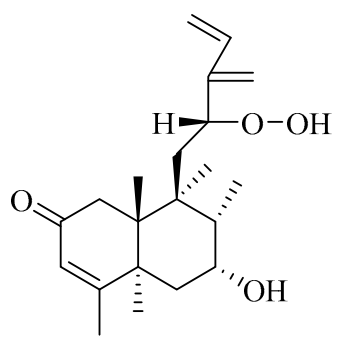

Stachaegyptin F (195)

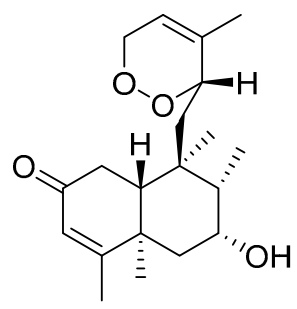

Stachaegyptin H (197)

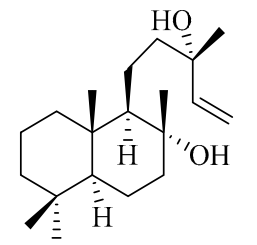

13-Epi-sclareol (200)

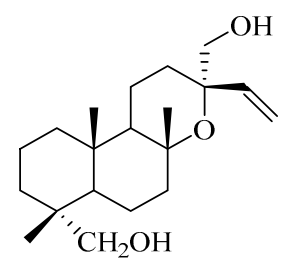

13-Epi-jabugodiol (202) 
Table 27. Cont.

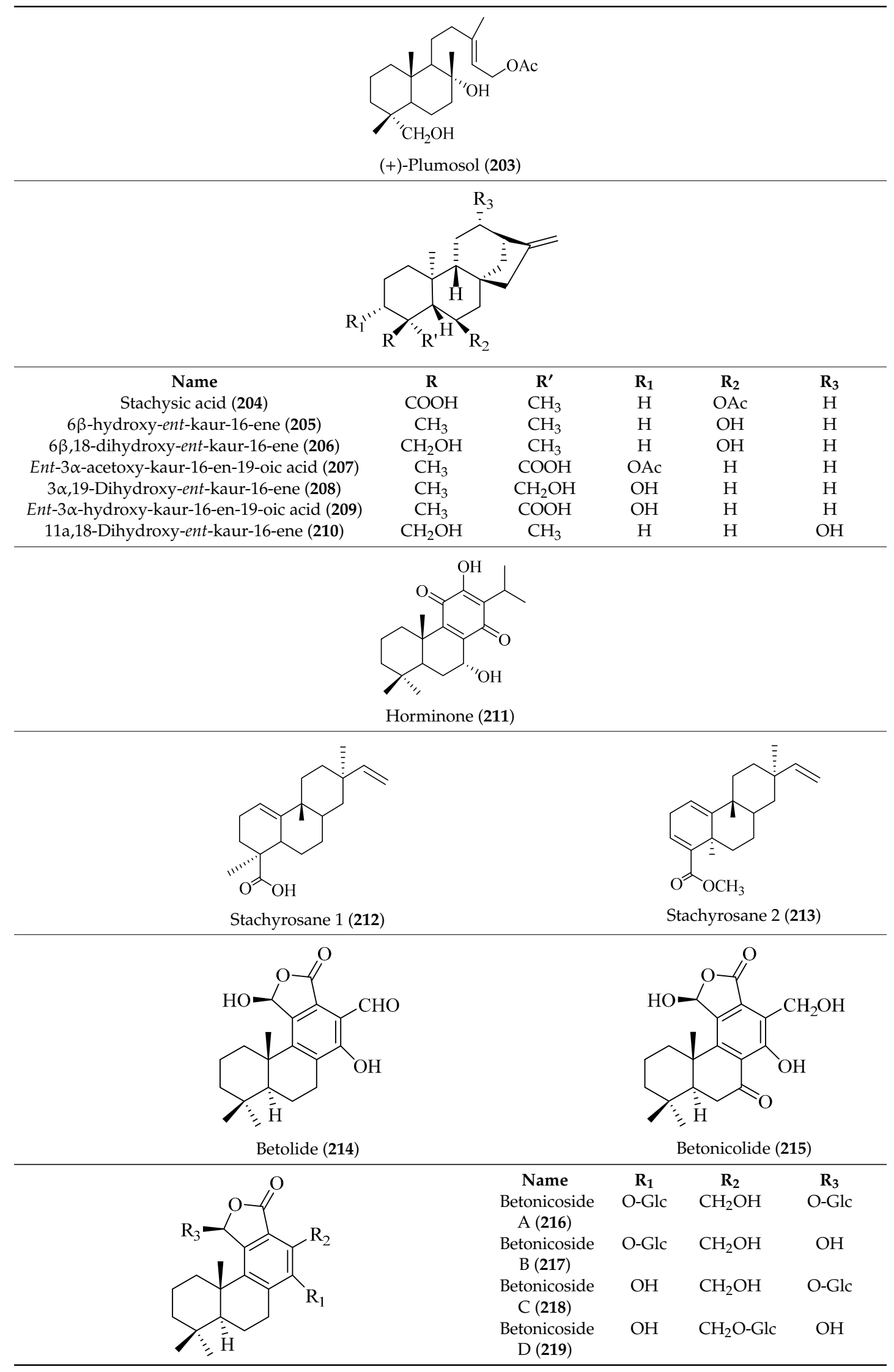

Glc: Glucose. 


\subsection{Triterpene Derivatives, Phytosterols and Phytoecdysteroids}

Triterpene derivatives and phytosterols are major secondary metabolites of Lamiaceae family. In genus Stachys, five phytosterol derivatives (220-224) were found in S. byzantina [17,35], S. annua [95], S. spinosa [99], S. tetragona [100], S. palustris [146] and S. alopecuros subsp. divulsa [119] (Tables 14 and 28). Furthermore, the triterpenoids; ursolic (226) and oleanolic (227) acids were only reported from the section Olisia (subg. Stachys) $[95,99,100]$. Kotsos et al. (2007) isolated an oleanolic lactone derivative (228) of the aerial parts of S. spinosa [99]. It is noteworthy to be mentioned the presence of saponin derivatives in genus Stachys (Tables 14 and 28). The first saponins isolated from this genus were from the water extract of the whole plant of S. riederi, including 8 stachyssaponins (I-VIII, 231-238) [147]. Afterwards, stachyssaponins A-B (229-230) were found from the methanol extract of the aerial parts of S. parviflora [63].

Few Stachys spp. include phytoecdysteroids (Tables 14 and 28). Ramazanov and co-workers (2016) isolated five phytoecdysteroids from S. hissarica [67], namely 20-hydroxyecdysone (239), polipodin B (240), integristeron A (241), 2-desoxy-20-hydroxyecdysone (242) and 2-desoxyecdyson (243). 
Table 28. Triterpene derivatives, Phytosterols and Phytoecdysteroids from Stachys spp.

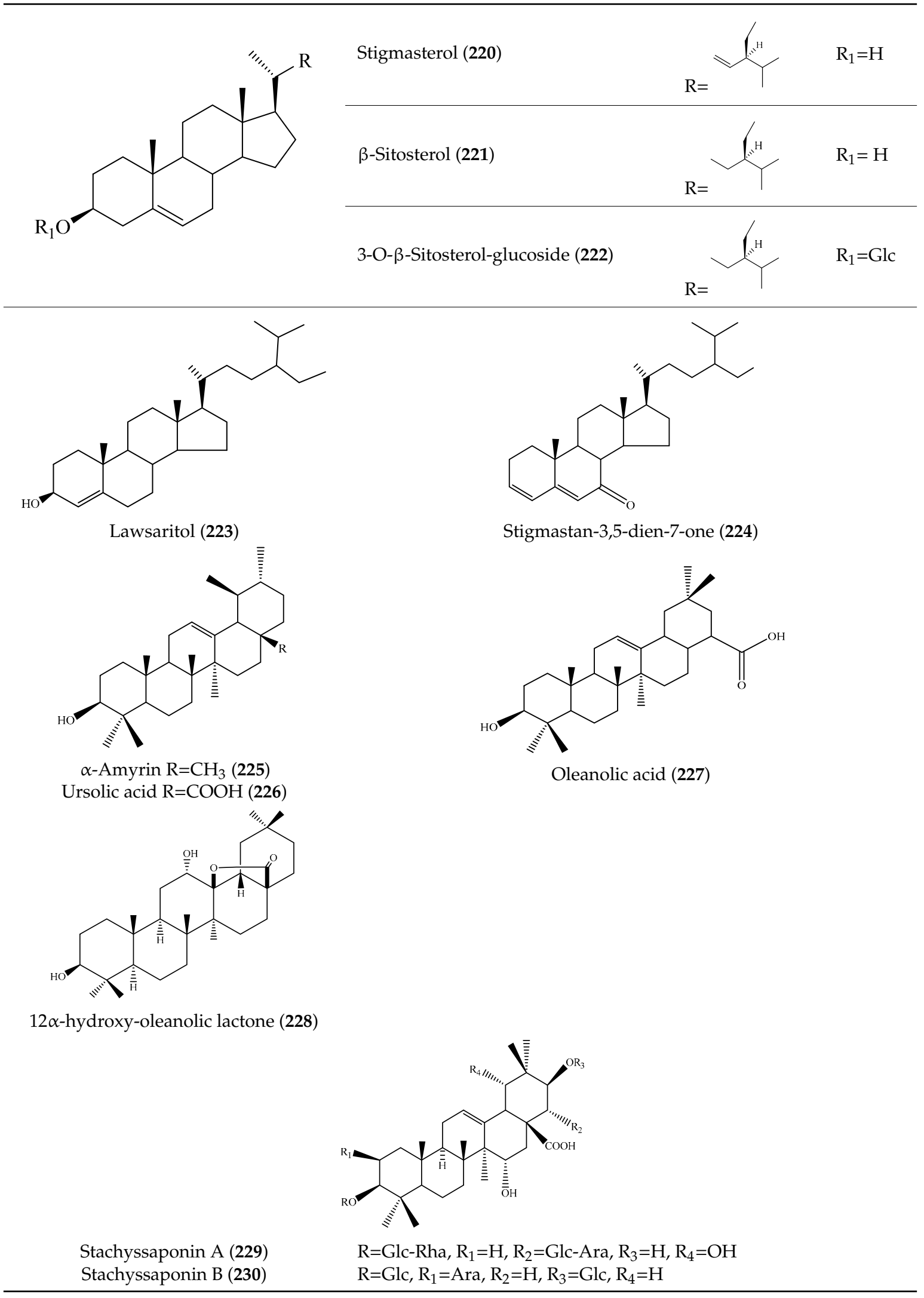


Table 28. Cont.

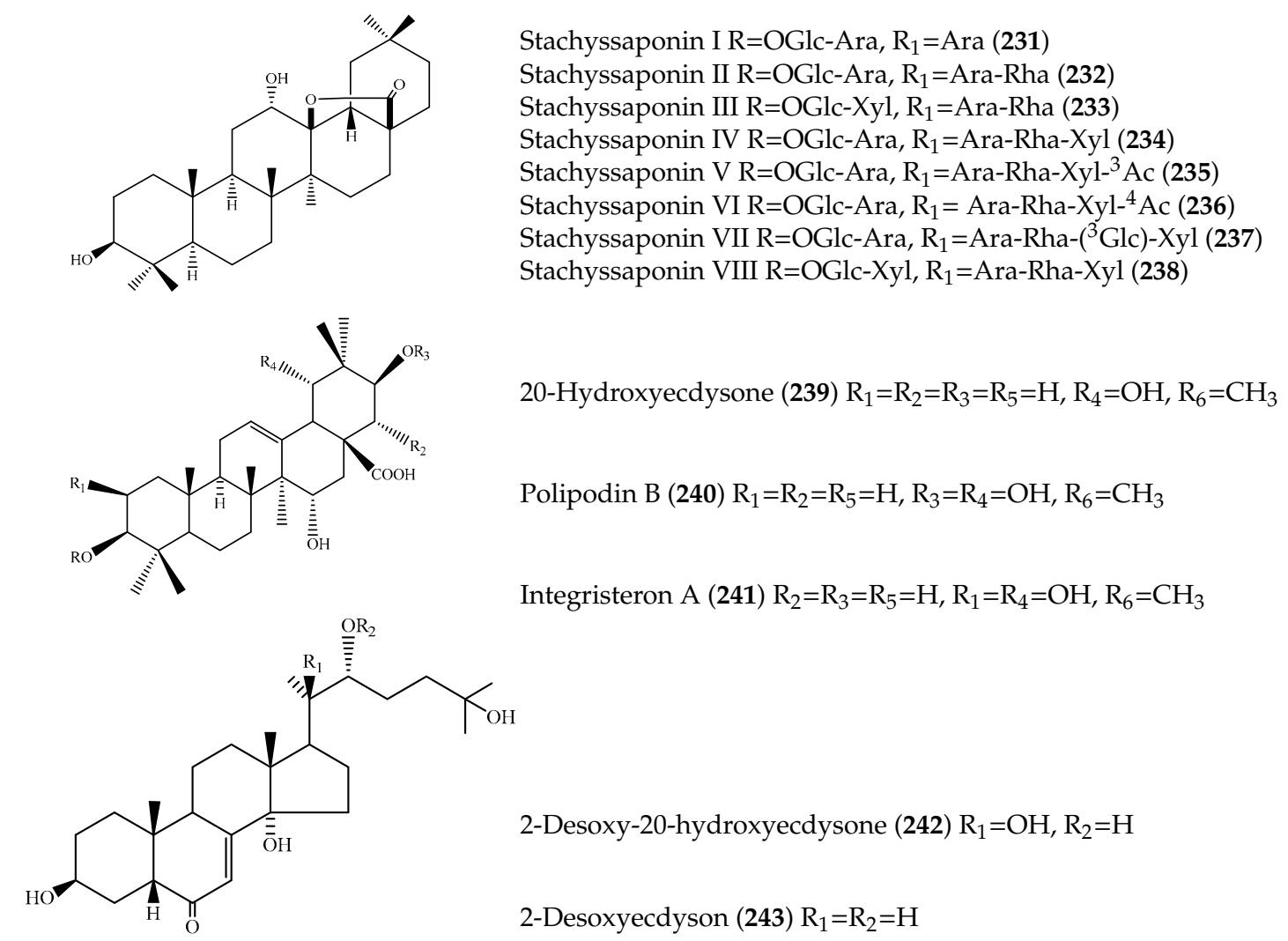

Glc: Glucose, Xyl: Xylose, Rha: Rhamnose, Ara: Arabinose.

\subsection{Other Chemical Categories}

Notable among the above-mentioned classes of compounds are the megastigmane derivatives from Stachys spp. (Tables 15 and 29). Takeda and colleagues (1997) isolated from the aerial parts of $S$. byzantina five bioactive compounds from this group, including byzantionosides A-B $(244,245)$, icariside B2 (246), (6R, 9R)- and (6R, 9S)-3-oxo- $\alpha$-ionol glucosides (247) and blumeol C glucoside (248) [148]. Furthermore, vomifoliol (249) and dehydrovomifoliol (250) were reported from the aerial parts of S. lanata, while citroside A (251) was isolated from the roots of this species [82]. This study also mentioned the presence of sugar ester (cistanoside F) from the roots of S. lanata [82]. At this point, we should note that few studies reported some oligosaccharides from Stachys spp. [3]. For instance, stachyose is a tetrasaccharide which consists one of the most common oligosaccharides in genus Stachys and shows beneficial effects for the gastrointestinal system as it can be directly consumed $[3,23,119,150]$. Precisely, the species S. sieboldii is a major source of this constituent $[27,151,152]$. Stachyose is an oligosaccharide, which can be directly consumed for the benefit of gastrointestinal system [150]. Furthermore, Yin and colleagues (2006) mentioned that the bitter taste of some Stachys species, such as S. annua and S. balansae, might be attributed to their bitter diterpene derivatives, like stachylone [22,151]. 
Table 29. Chemical structures of megastigmane derivatives from Stachys spp.

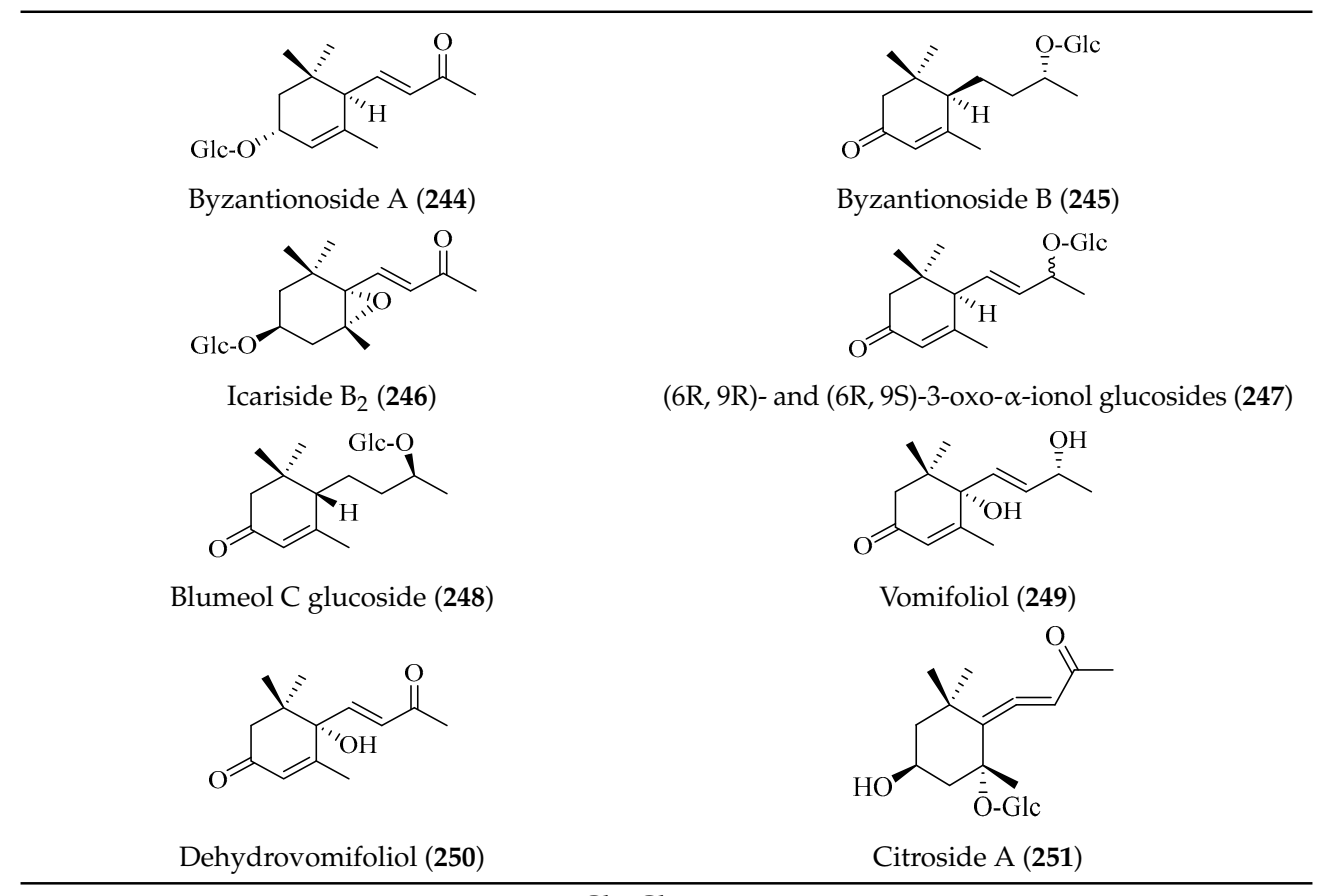

Glc: Glucose.

\section{Pharmacological Activities}

This section includes the most interesting pharmacological data of the last five years (from 2015 to 2020). Many studies exemplified the great antimicrobial, antioxidant and cytotoxic effects of the essential oils of these plants $[3,15]$. Tundis et al. (2014) described in detail the biological studies (in vitro and in vivo) of the essential oils, extracts and compounds [3]. Thus, in the present review, we focused on the current available pharmacological researches of the extracts and isolated compounds from Stachys spp. as they are presented in Table 30. 
Table 30. Pharmacological activities of Stachys spp.

\begin{tabular}{|c|c|c|c|}
\hline Species & Extract or Compound & Activity $^{a}$ & Ref \\
\hline S. aegyptiaca Pers. & Stachysolon diacetate (180) & $\begin{array}{c}\text { Cytotoxicity } \\
\text { HepG2 cell line } \mathrm{IC}_{50}: 59.5 \mu \mathrm{M}\end{array}$ & [132] \\
\hline \multirow{2}{*}{ S. affinis Bunge (=S. sieboldii Miq.) } & Ethyl acetate fraction & $\begin{array}{c}\text { Antioxidant } \\
\text { DPPH IC } 50: 0.85 \pm 0.04 \mu \mathrm{g} / \mathrm{mL} \\
\text { Superoxide radical scavenging activity: } 38.63-61.41 \% \\
\end{array}$ & [28] \\
\hline & Ethanol & $\begin{array}{l}\text { Cytotoxicity } \\
\text { K562 cell line; SH-SY5Y cell line; Caco-2 cell line: } \text { n.a. } \\
\text { Anti-ROS } \\
\text { K562 cell line; SH-SY5Y cell line; Caco-2 cell line } \mathrm{EC}_{50}: 0.0023 \mathrm{mg} / \mathrm{mL} ; 0.05 \mathrm{mg} / \mathrm{mL} ; 0.026 \mathrm{mg} / \mathrm{mL}\end{array}$ & [27] \\
\hline \multirow{3}{*}{ S. byzantina K. Koch. } & Methanol & $\begin{array}{c}\text { Antioxidant } \\
\text { Phosphomolybdenum (mmol TEs/g extract): } 1.49 \pm 0.12 \\
\text { ABTS (mg TEs/g extract): } 143.85 \pm 0.47 \\
\text { Nitric oxide (mmol TEs/g extract): } 1.84 \pm 0.02 \\
\text { CUPRAC (mg TEs/g extract): } 134.73 \pm 10.37\end{array}$ & \multirow{3}{*}[153]{} \\
\hline & Water & $\begin{array}{l}\text { DPPH (mg TEs/g extract): } 125.26 \pm 1.47 \\
\text { Superoxide anion }(\mathrm{mg} \text { TEs/g extract): } 50.68 \pm 2.05 \\
\text { FRAP (mg TEs/g extract): } 98.73 \pm 2.14 \\
\text { Chelating effect (mg EDTAEs/g extract): } 16.69 \pm 0.96\end{array}$ & \\
\hline & Ethyl acetate & 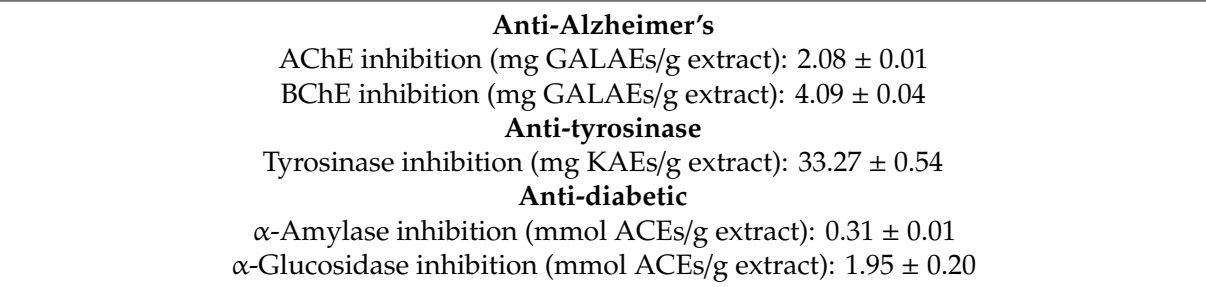 & \\
\hline
\end{tabular}


Table 30. Cont.

\begin{tabular}{|c|c|c|c|}
\hline Species & Extract or Compound & Activity $^{a}$ & Ref \\
\hline \multirow{5}{*}{ S. cretica L. subsp. smyrnaea Rech. f. } & Methanol & $\begin{array}{c}\text { Antioxidant } \\
\text { Ferrous ion chelating (mg EDTAEs/g dp): } 4.82 \pm 0.04 \\
\text { Phosphomolybdenum (mg TEs/g dp): } 71.94 \pm 4.56 \\
\text { DPPH (mg TEs/g dp): } 9.10 \pm 0.04 \\
\text { ABTS (mg TEs/g dp): } 17.36 \pm 0.07 \\
\text { CUPRAC (mg TEs/g dp): } 14.67 \pm 0.02 \\
\text { FRAP (mg TEs/g dp): } 12.98 \pm 0.11\end{array}$ & \multirow{5}{*}{ [81] } \\
\hline & Methanol & $\begin{array}{c}\text { Anti-Alzheimer's } \\
\text { AChE inhibition ( } \mu \mathrm{g} \text { GALAEs/g dp): } 343.78 \pm 10.79\end{array}$ & \\
\hline & Ethyl acetate & BChE inhibition ( $\mu \mathrm{g}$ GALAEs/g dp): $167.68 \pm 2.68$ & \\
\hline & Ethyl acetate & $\begin{array}{c}\text { Anti-tyrosinase } \\
\text { Tyrosinase inhibition (mg KAEs/g dp): } 2.45 \pm 0.05\end{array}$ & \\
\hline & Methanol & $\begin{array}{c}\text { Anti-diabetic } \\
\alpha \text {-Amylase inhibition (mg ACEs/g dp): } 61.47 \pm 0.05 \\
\alpha \text {-Glucosidase inhibition (mg ACEs/g dp): } 47.84 \pm 0.78\end{array}$ & \\
\hline \multirow{7}{*}{$\begin{array}{l}\text { S. cretica L. subsp. mersinaea (Boiss.) } \\
\text { Rech. f. }\end{array}$} & Water & $\begin{array}{c}\text { Antioxidant } \\
\text { Phosphomolybdenum (mmol TEs/g extract): } 2.17 \pm 0.21 \\
\text { DPPH (mg TEs/g extract): } 176.21 \pm 2.52\end{array}$ & \multirow{7}{*}{ [108] } \\
\hline & Methanol & $\begin{array}{c}\text { ABTS (mg TEs/g extract): } 292.67 \pm 1.53 \\
\text { CUPRAC (mg TEs/g extract): } 256.79 \pm 2.02 \\
\text { FRAP (mg TEs/g extract): } 236.44 \pm 2.96 \\
\text { Ferrous ion chelating (mg EDTAEs/g extract): } 18.57 \pm 0.04\end{array}$ & \\
\hline & Methanol & $\begin{array}{c}\text { Anti-Alzheimer's } \\
\text { AChE inhibition (mg GALAEs/g extract): } 2.03 \pm 0.15\end{array}$ & \\
\hline & Ethyl acetate & BChE inhibition (mg GALAEs/g extract): $0.39 \pm 0.01$ & \\
\hline & Ethyl acetate & $\begin{array}{c}\text { Anti-tyrosinase } \\
\text { Tyrosinase inhibition (mg KAEs/g extract): } 16.58 \pm 0.31\end{array}$ & \\
\hline & Ethyl acetate & $\begin{array}{c}\text { Anti-diabetic } \\
\alpha \text {-Amylase inhibition (mg ACEs/g extract): } 396.50 \pm 4.63\end{array}$ & \\
\hline & Methanol & $\alpha$-Glucosidase inhibition (mg ACEs/g extract): $734.47 \pm 4.32$ & \\
\hline
\end{tabular}


Table 30. Cont.

\begin{tabular}{|c|c|c|c|}
\hline Species & Extract or Compound & Activity $^{a}$ & Ref \\
\hline \multirow{4}{*}{ S. cretica L. subsp. vacillans Rech. f. } & Methanol & $\begin{array}{c}\text { Antioxidant } \\
\text { (mg TE/g extract) } \\
\text { DPPH: } 191.47 \pm 5.77 \\
\text { ABTS: } 213.93 \pm 21.83 \\
\text { CUPRAC: } 579.23 \pm 13.99 \\
\text { FRAP: } 254.40 \pm 8.58\end{array}$ & \multirow{4}{*}{ [112] } \\
\hline & Water & Ferrous ion chelating (mg EDTAE/g extract): $68.72 \pm 0.80$ & \\
\hline & Methanol & $\begin{array}{c}\text { Anti-tyrosinase } \\
\text { Tyrosinase inhibition (mg KAE/g extract): } 314.04 \pm 2.05\end{array}$ & \\
\hline & Methanol & $\begin{array}{l}\text { Anti-diabetic } \\
\alpha \text {-Amylase inhibition (mg ACE/g extract): } 433.99 \pm 5.10\end{array}$ & \\
\hline S. ehrenbergii Boiss. & Methanol & $\begin{array}{c}\text { Antioxidant } \\
\text { ABTS IC }_{50}: 52 \pm 7.5 \mathrm{mg} / \mathrm{mL} \\
\text { Cytotoxicity } \\
\text { A549 cell line } \mathrm{IC}_{50}: 420 \pm 104 \mu \mathrm{g} / \mathrm{mL}\end{array}$ & [154] \\
\hline S. glutinosa L. & Dichloromethane; Xanthomicrol (69) & $\begin{array}{c}\text { Opioid Receptors binding affinity (in silico) } \\
\mathrm{K}_{\mathrm{i}} \text { for } \mathrm{MOR}=10.3 \mu \mathrm{g} / \mathrm{mL}, \mathrm{K}_{\mathrm{i}} \text { for } \\
\mathrm{DOR}=9.0 \mu \mathrm{g} / \mathrm{mL} ; \mathrm{K}_{\mathrm{i}} \text { for } \mathrm{MOR}=0.83 \mu \mathrm{M}, \mathrm{K}_{\mathrm{i}} \text { for } \mathrm{DOR}=3.6 \mu \mathrm{M} \\
\text { Antinociceptive (in vivo) }\end{array}$ & [107] \\
\hline \multirow[t]{3}{*}{ S. guyoniana Noë ex Batt. } & $\begin{array}{l}\text { Chloroform } \\
n \text {-Butanol } \\
\text { Chloroform } \\
n \text {-Butanol }\end{array}$ & $\begin{array}{c}\text { Antioxidant } \\
\beta \text {-carotene IC } \text { IC }_{50}: 2.30 \pm 1.27 \mu \mathrm{g} / \mathrm{mL} \\
\text { DPPH IC }_{50}: 2.91 \pm 0.14 \mu \mathrm{g} / \mathrm{mL} \\
\text { ABTS IC } 50: 7.29 \pm 0.23 \mu \mathrm{g} / \mathrm{mL} \\
\text { CUPRAC A } 0.50: 0.15 \pm 0.05 \mu \mathrm{g} / \mathrm{mL} \\
\text { Metal chelating assay }(\%) \text { of inhibition at } 100 \mu \mathrm{g} / \mathrm{mL}: 48.00 \pm 1.71\end{array}$ & \multirow[t]{3}{*}{ [155] } \\
\hline & $n$-Butanol & $\begin{array}{l}\text { Anticholinesterase } \\
\text { AChE inhibition } \mathrm{IC}_{50}: 5.78 \pm 0.01 \mu \mathrm{g} / \mathrm{mL} \\
\text { BChE inhibition } \mathrm{IC}_{50}: 39.10 \pm 1.41 \mu \mathrm{g} / \mathrm{mL}\end{array}$ & \\
\hline & $\begin{array}{l}n \text {-Butanol; } \\
\text { Chloroform }\end{array}$ & $\begin{array}{l}\text { Antibacterial } \\
\text { MIC value: S. aureus } 32 \pm 0.90 \mu \mathrm{g} / \mathrm{mL} \text {, E. aerogenes } 32 \pm 0.70 \mu \mathrm{g} / \mathrm{mL} \text {; E. coli } 64 \pm 0.60 \mu \mathrm{g} / \mathrm{mL}\end{array}$ & \\
\hline S. hissarica Regel & - & Wound Healing (in vivo) & [67] \\
\hline
\end{tabular}


Table 30. Cont.

\begin{tabular}{|c|c|c|c|}
\hline Species & Extract or Compound & Activity $^{a}$ & Ref \\
\hline \multirow{3}{*}{$\begin{array}{c}\text { S. iberica var. densipilosa } \mathrm{R} . \\
\text { Bhattacharjee }\end{array}$} & Ethyl acetate; & $\begin{array}{c}\text { Antioxidant } \\
\text { ABTS (mg TEs/g extract): } 138.16 \pm 0.49 \\
\text { Nitric oxide (mmol TEs/g extract): } 1.81 \pm 0.01 \\
\text { Superoxide anion (mg TEs/g extract): } 41.31 \pm 1.64 \\
\text { CUPRAC (mg TEs/g extract): } 111.47 \pm 4.67\end{array}$ & \multirow{3}{*}{ [153] } \\
\hline & Water & $\begin{array}{c}\text { DPPH (mg TEs/g extract): } 82.52 \pm 1.62 \\
\text { FRAP (mg TEs/g extract): } 89.15 \pm 0.82 \\
\text { Chelating effect (mg EDTAEs/g extract): } 9.24 \pm 0.87\end{array}$ & \\
\hline & Ethyl acetate & 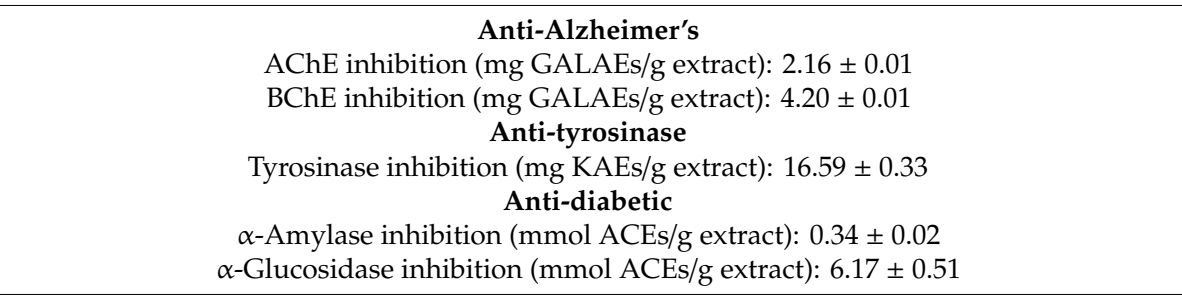 & \\
\hline S. iva Griseb. & Stachysetin (98) & $\begin{array}{c}\text { Anti-diabetic (in silico) } \\
\begin{array}{c}\text { Dipeptyl peptidase IV, peroxisome proliferator-active receptor gamma, aldose reductase, } \\
\text { glycogen kinase, pancreatic alpha amylase } \\
\text { precursor }\end{array}\end{array}$ & [56] \\
\hline \multirow[b]{2}{*}{ S. mialhesii Noé } & $\begin{array}{l}n \text {-Butanol; } \\
\text { Isoscutellarein-7-O-[6"'-O-acetyl]- } \beta \text {-D- } \\
\text { allopyranosyl-(1 } \rightarrow 2)-\beta \text {-D-glucoside (15) }\end{array}$ & $\begin{array}{c}\text { Antioxidant } \\
\text { DPPH IC } 50: 0.047 \pm 0.0048 \mathrm{mg} / \mathrm{mL} ; 0.066 \pm 0.002 \mathrm{mg} / \mathrm{mL}\end{array}$ & \multirow[b]{2}{*}{ [103] } \\
\hline & $n$-Butanol & $\begin{array}{c}\text { Acute toxicity (in vivo) } \\
\text { Not toxic (10 g/kg of extract) } \\
\text { Antinociceptive (in vivo) } \\
\text { Inhibition of the writhing response induced by acetic acid (dose: 10,000; } 5000 \mathrm{mg} / \mathrm{kg} \text { ) } 77.11 \% \text {, } \\
58.22 \% \\
\text { Antiinflammatory (in vivo) } \\
\text { Carrageenan-induced paw edema (dose: } 5000 \mathrm{mg} / \mathrm{kg} \text { ) } 52.03 \% \\
\text { Ulcerogenic (in vivo) } \\
\text { n.a. }\end{array}$ & \\
\hline S. mucronata Sieb. & $n$-Butanol fraction & Anti-radical & [156] \\
\hline
\end{tabular}


Table 30. Cont.

\begin{tabular}{|c|c|c|c|}
\hline Species & Extract or Compound & Activity $^{a}$ & Ref \\
\hline \multirow{6}{*}{ S. lavandulifolia Vahl. } & $\begin{array}{l}\text { Methanol Soxhlet extract; Arbutin (107), } \\
\text { Ethanol; Arbutin (107), } \\
\text { Methanol Soxhlet extract; Arbutin (107), }\end{array}$ & $\begin{array}{c}\text { Antioxidant } \\
\text { DPPH IC }_{50}: 25.0 \pm 1.1 \mu \mathrm{g} / \mathrm{mL} ; 62.5 \pm 0.9 \mu \mathrm{g} / \mathrm{mL} \\
\text { ABTS IC } \mathrm{I}_{50}: 19.9 \mu \mathrm{g} / \mathrm{mL} ; 45.7 \mu \mathrm{g} / \mathrm{mL} \\
\text { FRAP }(\mu \mathrm{M} \text { Fe(II)/g): } 44.5 \pm 1.0 ; 12.2 \pm 0.6\end{array}$ & \multirow{4}{*}{116} \\
\hline & Methanol; Ethanol & $\begin{array}{c}\beta \text {-carotene } \mathrm{IC}_{50}: 29.3 \mu \mathrm{g} / \mathrm{mL}(30 \mathrm{~min}), 60.3 \mu \mathrm{g} / \mathrm{mL}(60 \mathrm{~min}) ; 33.0 \mu \mathrm{g} / \mathrm{mL}(30 \mathrm{~min}), 34.6 \mu \mathrm{g} / \mathrm{mL} \text { (60 } \\
\end{array}$ & \\
\hline & Ethanol & $\begin{array}{c}\text { Anti-tyrosinase } \\
\text { Tyrosinase inhibition } \mathrm{IC}_{50}: 33.4 \pm 0.8 \mu \mathrm{g} / \mathrm{mL}\end{array}$ & \\
\hline & $\begin{array}{l}\text { Hexane } \\
\text { Dichloromethane }\end{array}$ & 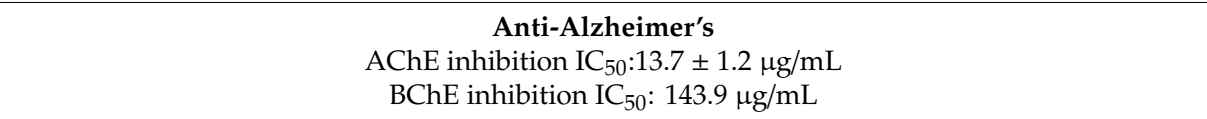 & \\
\hline & Chloroform & $\begin{array}{c}\text { Cytotoxicity } \\
\text { Brine Shrimp lethality test: } 121.8 \pm 5.6 \mu \mathrm{g} / \mathrm{mL}\end{array}$ & \multirow{2}{*}{ [13] } \\
\hline & Apigenin (1); Chrysosplenetin (84) & $\begin{array}{l}\text { MRC-5 cell line IC } \text { I0 }_{50}: 35.67 \mu \mathrm{g} / \mathrm{mL} ; \text { MDA-MB-231 cell line } \mathrm{IC}_{50}: 88.23 \mu \mathrm{g} / \mathrm{mL}, \mathrm{HT}-29 \text { cell line } \mathrm{IC}_{50} \text { : } \\
116.50 \mu \mathrm{g} / \mathrm{mL}\end{array}$ & \\
\hline $\begin{array}{l}\text { S. officinalis (L.) Trevis } \\
\text { (=Betonica officinalis L.) }\end{array}$ & $\begin{array}{l}\text { Acetone } \\
\text { Methanol }\end{array}$ & Genotoxicity & [157] \\
\hline S. ocymastrum (L.) Briq. (=S. hirta L.) & 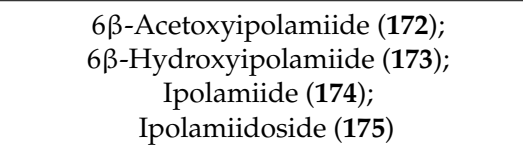 & Antiangiogenic (in vivo) & [123] \\
\hline $\begin{array}{l}\text { S. parviflora Benth. } \\
\text { (=Phlomidoschema parviflorum } \\
\text { (Benth.) Vved.) }\end{array}$ & Methanol & $\begin{array}{c}\text { Antioxidant } \\
\text { DDPH IC } 50: 76.87 \pm 0.57 \mu \mathrm{g} / \mathrm{mL} \\
\text { BCB IC } \text { I50 }_{50}: 188.47 \pm 0.76 \mu \mathrm{g} / \mathrm{mL} \\
\text { Cytotoxicity } \\
\text { A2780 cell line } \mathrm{IC}_{50}: \mathrm{n} . \mathrm{a} \\
\text { HCT cell line IC } \mathrm{IC}_{50}: \mathrm{n} . \mathrm{a} \\
\text { B16F10 cell line IC } \mathrm{I}_{50}: \mathrm{n} . \mathrm{a} \\
\text { Antibacterial } \\
\text { MIC: Bacillus cereus } 0.12 \mathrm{mg} / \mathrm{mL}\end{array}$ & [64] \\
\hline
\end{tabular}


Table 30. Cont.

\begin{tabular}{|c|c|c|c|}
\hline Species & Extract or Compound & Activity $^{a}$ & Ref \\
\hline \multirow{6}{*}{ S. pilifera Benth. } & Terpenoid fraction & $\begin{array}{c}\text { Cytotoxicity } \\
\text { HT29 cell line IC } \text { IC }_{50}: 46.44 \mu \mathrm{g} / \mathrm{mL}\end{array}$ & \multirow{2}{*}{ [45] } \\
\hline & $\begin{array}{l}70 \% \text { Methanol } \\
\text { Alkaloid fraction }\end{array}$ & $\begin{array}{c}\text { Antiproliferative } \\
\text { Caspase-8 increased 99\% } \\
\text { Caspase-9 increased } 85.38 \%\end{array}$ & \\
\hline & $70 \%$ Ethanol & Hepatoprotective (in vivo) & [158] \\
\hline & Hydroalcoholic & $\begin{array}{c}\text { Antioxidant (in vivo) } \\
\text { Hepatoprotective (in vivo) }\end{array}$ & [159] \\
\hline & Hydroalcoholic & $\begin{array}{c}\text { Antioxidant (in vivo) } \\
\text { Renoprotective (in vivo) }\end{array}$ & [19] \\
\hline & Water & Neuroprotective (in vivo) & [152] \\
\hline S. riederi var. japonica (Miq.) H. Hara & $80 \%$ Ethanol & $\begin{array}{c}\text { Antioxidant/Cytoprotective } \\
\text { UVA-irradiated human dermal fibroblasts (HDFs) } \\
\text { Cytotoxicity } \\
\text { HDFs: l.a./n.a }\end{array}$ & [160] \\
\hline \multirow[t]{3}{*}{ S. sieboldii Miq. (=S. affinis Bunge) } & $\begin{array}{c}n \text {-Hexane fraction } \\
n \text {-Hexane; } 85 \% \text { MeOH; } n \text {-BuOH; } \\
\text { water fractions }\end{array}$ & $\begin{array}{c}\text { Antioxidant } \\
\text { ROS inhibition: } 63 \% \\
\text { Increased GSH levels } \\
\text { Inhbited oxidative DNA damage }>90 \%\end{array}$ & [29] \\
\hline & (Root powder) & $\begin{array}{c}\text { Anti-obesity (in vivo) } \\
\text { Anti-dyslipidemic (in vivo) }\end{array}$ & [161] \\
\hline & $20 \%$ Ethanol & Memory protective (in vivo) & [162] \\
\hline S. sylvatica $\mathrm{L}$. & Hydroalcoholic & $\begin{array}{c}\text { Polycystic ovary syndrome (in vivo) }(500 \mathrm{mg} / \mathrm{kg})(\mathrm{mIU} / \mathrm{mL}) \\
\text { FSH } 5.95 \pm 0.02 \mathrm{mIU} / \mathrm{mL} \\
\text { LH } 6.48 \pm 0.09 \mathrm{mIU} / \mathrm{mL} \\
\text { Estrogen } 0.9 \pm 0.07 \mathrm{mIU} / \mathrm{mL} \\
\text { LH/FSH } 6.48 / 5.59 \mathrm{mIU} / \mathrm{mL}\end{array}$ & [47] \\
\hline
\end{tabular}


Table 30. Cont.

\begin{tabular}{|c|c|c|c|}
\hline Species & Extract or Compound & Activity $^{a}$ & Ref \\
\hline \multirow{4}{*}{ S. thirkei K. Koch. } & Methanol & $\begin{array}{c}\text { Antioxidant } \\
\beta \text {-carotene IC } \mathrm{IC}_{50}: 47.79 \pm 0.59 \mu \mathrm{g} / \mathrm{mL} \\
\text { DPPH IC } 50: 49.31 \pm 0.38 \mu \mathrm{g} / \mathrm{mL} \\
\text { ABTS IC } 50: 13.34 \pm 0.02 \mu \mathrm{g} / \mathrm{mL} \\
\text { CUPRAC absorbance } \%: 1.88 \pm 0.02\end{array}$ & \multirow{4}{*}{ [84] } \\
\hline & Acetone & $\begin{array}{c}\text { Anticholinesterase } \\
\text { AChE inhibition } \mathrm{IC}_{50}: 52.46 \pm 1.26 \% \\
\text { BChE inhibition } \mathrm{IC}_{50}: 75.04 \pm 1.91 \%\end{array}$ & \\
\hline & Methanol & $\begin{array}{c}\text { Cytotoxicity } \\
\text { A549 and L929 Fibroblast cells (100 mg/mL): n.a. }\end{array}$ & \\
\hline & Acetone; Methanol & 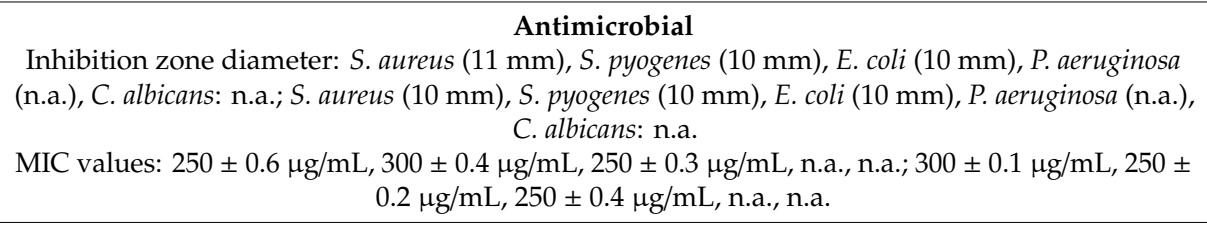 & \\
\hline S. tmolea Boiss. & Water & $\begin{array}{c}\text { Antioxidant } \\
\text { DPPH (mg TEs/g dp): } 50.88 \pm 1.55 \\
\text { ABTS (mg TEs/g dp): } 44.39 \pm 3.24 \\
\text { CUPRAC (mg TEs/g dp): } 87.57 \pm 0.83 \\
\text { FRAP (mg TEs/g dp): } 51.80 \pm 2.17 \\
\text { Phosphomolybdenum (mg TEs/g dp): } 40.58 \pm 3.45 \\
\text { Ferrous ion chelating (mg EDTAEs/g dp): } 1.10 \pm 0.03\end{array}$ & [85] \\
\hline
\end{tabular}

${ }^{\text {a }}$ Only the highest activity; n.a.:no activity; l.a.: low activity. 


\subsection{Antioxidant Activity/Cytoprotective}

Tundis et al. (2015) evaluated five extracts ( $n$-hexane, dichloromethane, methanol, methanol with Soxhlet apparatus and ethanol 70\% extract) from the aerial parts of $S$. lavandulifolia for their antioxidant activity, using $\beta$-carotene bleaching test, 2,2'-azino-bis(3-ethylbenzothiazoline-6-sulphonic acid (ABTS), 1,1-Diphenyl-2-picrylhydrazyl (DPPH), and Ferric Reducing Antioxidant Power (FRAP) assays [116]. The most polar extracts, ethanol $70 \%$ and methanol extracts, showed the highest radical scavenging activity against ABTS radical ( $\mathrm{IC}_{50}$ values of 19.9 and $22.8 \mu \mathrm{g} / \mathrm{mL}$, respectively), whereas the methanol extract Soxhlet apparatus was the most active in the DPPH method ( $\mathrm{IC}_{50}$ of $25.0 \mu \mathrm{g} / \mathrm{mL}$ ). In the $\beta$-carotene bleaching test, the methanol and ethanol extract demonstrated the stronger activity after $30 \mathrm{~min}$ of incubation $\left(\mathrm{IC}_{50}=29.3\right.$ and $33.0 \mu \mathrm{g} / \mathrm{mL}$, respectively) and the $\mathrm{IC}_{50}$ values were of 60.3 and $34.6 \mu \mathrm{g} / \mathrm{mL}$, respectively after $60 \mathrm{~min}$ of incubation. Moreover, they studied the antioxidant activity of bioactive secondary metabolites; arbutin (107), acteoside (118), monomelittoside (165), melittoside (166), 5-allosyloxy-aucubin (167), and stachysolone (177), reporting that in both DPPH and ABTS assays the most active compounds was arbutin (107) with $\mathrm{IC}_{50}$ values of 62.5 and $45.7 \mu \mathrm{g} / \mathrm{mL}$, respectively [116]. Another work investigated the antioxidant activity of three extracts of S. guyoniana, through $\beta$-carotene-linoleic acid, DPPH, ABTS, CUPric Reducing Antioxidant Capacity (CUPRAC) and metal chelating assays [155]. The chloroform extract had the highest antioxidant activity $\left(\mathrm{IC}_{50}=2.3 \pm 1.27 \mu \mathrm{g} / \mathrm{mL}\right)$ in $\beta$-carotene-linoleic acid and in ABTS method $\left(\mathrm{IC}_{50}=7.29 \pm 0.23 \mu \mathrm{g} / \mathrm{mL}\right)$. The $n$-butanol extract showed the better antioxidant capacity in DPPH test $\left(\mathrm{IC}_{50}=2.91 \pm 0.14 \mu \mathrm{g} / \mathrm{mL}\right)$ compared to other extracts and to the reference compound $\alpha$-tocopherol $\left(\mathrm{IC}_{50}=7.31 \pm 0.17 \mu \mathrm{g} / \mathrm{mL}\right.$ ), as well as it demonstrated highest activity in CUPRAC method $\left(\mathrm{A}_{0.50}=0.15 \pm 0.05 \mu \mathrm{g} / \mathrm{mL}\right)$ and in metal cheating assay (inhibition at $100 \mu \mathrm{g} / \mathrm{mL}$ : 48\%). In addition, Laggoune et al. (2016) demonstrated the great antioxidant properties in vivo of S. mialhesii [103]. Particularly, the $n$-butanol extract of the specific plant showed $\mathrm{IC}_{50}$ value of $0.047 \mathrm{mg} / \mathrm{mL}$ in $\mathrm{DPPH}$ assay, while the $\mathrm{IC}_{50}$ value of the isolated compound isoscutellarein-7-O-[6"' -O-acetyl]- $\beta$-D-allopyranosyl-(1 $\rightarrow 2)-\beta$-D-glucoside (15) was $0.066 \mathrm{mg} / \mathrm{mL}$ and the reference compound quercetin was $0.012 \mathrm{mg} / \mathrm{mL}$. Notably, they also mentioned that the extract (up to $10 \mathrm{~g} / \mathrm{kg}$ ) did not show any toxicity in mice during $24 \mathrm{~h}$ after administration. In addition, the antioxidant activity of three subspecies of $S$. cretica (i.e., S. cretica subsp. mersinaea; S. cretica subsp. smyrnaea; S. cretica subsp. vacillans) were investigated in different works $[81,108,112]$. The antioxidant capacity of the methanol extract of $S$. parviflora was measured, exhibiting an $\mathrm{IC}_{50}$ value of $76.87 \pm 0.57 \mu \mathrm{g} / \mathrm{mL}$ (DPPH method) and of $188.47 \pm 0.76 \mu \mathrm{g} / \mathrm{mL}$ ( $\beta$-carotene bleaching test; $\mathrm{BCB}$ ), while the standard compound, butylated hydroxytoluene (BHT), had stronger activity in both tests (DPPH test: $\mathrm{IC}_{50}=1.23 \pm 0.02 \mu \mathrm{g} / \mathrm{mL}$; BCB test: $34.31 \pm 0.40 \mu \mathrm{g} / \mathrm{mL}$ ) [64]. Guo et al. (2018) examined the antioxidant activity of five fractions from the $70 \%$ ethanol extract of tubers of S. affinis by DPPH assay and superoxide radical scavenging activity [28]. The ethyl acetate fraction showed extremely high antioxidant activity in DPPH method $\left(\mathrm{IC}_{50}=0.85 \pm 0.04 \mu \mathrm{g} / \mathrm{mL}\right)$ with $\alpha$-tocopherol as positive control $\left(\mathrm{IC}_{50}=18.68 \pm 0.51 \mu \mathrm{g} / \mathrm{mL}\right)$. They reported that this great antioxidant activity was attributed to the high content in phenolics and flavonoids of this fraction and confirmed the use of this plant as a natural antioxidant. Another work studied the antioxidant activity of the extracts and fractions of the same Stachys species on reactive oxygen species (ROS) production induced by $\mathrm{H}_{2} \mathrm{O}_{2}$ in HT-1080 cells [29]. In particular, the $n$-hexane fraction decreased $\mathrm{H}_{2} \mathrm{O}_{2}$-induced ROS and oxidative stress-induced DNA damage, as well as it increased glutathione (GSH) production. The species S. mucronata demonstrated strong anti-radical activity due to the high content in polyphenols [156]. A recent study conducted by Aminfar et al. (2019) described a chemometric-based approach in order to classify S. lanata by Gas Chromatography-Mass Spectrometry (GC-MS) fingerprints and to correlate their chemical constituents with their antioxidant capacity [35]. They identified eight antioxidant markers which could also serve as volatile markers. In addition, Elfalleh and co-workers (2019) demonstrated the differences of the antioxidant properties of the extracts of $S$. tmolea, reporting that water extract exhibited highest activity than methanol extract, using DPPH, ABTS, CUPRAC, FRAP, phosphomolybdenum and ferrous ion chelating methods [85]. A survey 
conducted by Hwang et al. (2019) demonstrated that the ethanol extract of S. riederi var. japonica exhibited antioxidant effects on ultraviolet A (UVA)-irradiated human dermal fibroblasts (HDFs), through suppression of ROS generation [160]. The antioxidant activity of the methanol extract of the Lebanese species S. ehrenbergii was measured by ABTS radical cation decolorization assay and the methanol extract showed an $\mathrm{IC}_{50}$ value of $52 \pm 7.5 \mathrm{mg} / \mathrm{mL}$ [154]. Furthermore, the chemical profile and some biological activities of three herbal teas in Anatolia were examined [84]. Among them, the methanol extract of $S$. thirkei showed strongest antioxidant capacity, through $\beta$-carotene $\left(\mathrm{IC}_{50}=47.79 \pm 0.59 \mu \mathrm{g} / \mathrm{mL}\right), \mathrm{DPPH}\left(\mathrm{IC}_{50}=49.31 \pm 0.38 \mu \mathrm{g} / \mathrm{mL}\right), \mathrm{ABTS}\left(\mathrm{IC}_{50}=13.34 \pm 0.02 \mu \mathrm{g} / \mathrm{mL}\right)$ and CUPRAC (absorbance\%: $1.88 \pm 0.02 \mu \mathrm{g} / \mathrm{mL}$ ) assays. Sadeghi et al. (2020) assessed the the antioxidant properties of hydroalcoholic extract of $S$. pilifera on nephrotoxicity induced with cisplatin (CP) in vivo (in rats), showing that the specific extract restored the antioxidant capacity, as well as it had renoprotective activity [19].

\subsection{Cytotoxicity and Antiproliferative Activity}

Venditti et al., (2017) investigated the cytotoxic activity and the anti-reactive oxygen species activity of the ethanol extract from tubers of the Chinese artichock (S. affinis) [27]. Regarding the cytotoxicity, the specific extract didn't demonstrate any activity in K562, SH-SY5Y and Caco-2 cell lines, even at the highest concentrations $(1.0 \mathrm{mg} / \mathrm{mL})$. The cytotoxic activity of extracts and isolated flavonoids from the aerial parts of $S$. lavandulifolia were studied by Delnavazi et al. (2018) through the 3-(4,5-dimethylthiazol-2-yl)-2,5-diphenyltetrazolium bromide (MTT) assay [13]. The dichloromethane extract showed the highest cytotoxic activity in brine shrimp lethality test $(\mathrm{BSLT})\left(\mathrm{LD}_{50}=121.8 \pm 5.6 \mu \mathrm{g} / \mathrm{mL}\right)$, while as a positive control was used podophyllotoxin $\left(\mathrm{LD}_{50}=3.1 \pm 0.6 \mu \mathrm{g} / \mathrm{mL}\right)$. Afterwards, they explored the cytotoxic activity of isolated flavonoids in three cancer cell lines (MDA-MB-231, HT-29 and MRC-5), using as reference compound tamoxifen. All the nine isolated flavonoids moderated the cytotoxicity activated on the studied cell lines. However, chrysosplenetin (84) was reported as the most active compound in the first two cell lines. In MRC-5 cell line, apigenin (1) exhibited the greatest activity. It is remarkable to point out that the specific study also mentioned the selective activity against cancer cells, reporting that chrysosplenetin (84), kumatakenin (79) and viscosine (78) exhibited higher selective toxicity against MDA-MB-231 cell line than tamoxifen. At this point, we should underlie that the great cytotoxic activity of these compounds is attributed to their substitutions with (poly)-methylated groups which increase this effect. Another study evaluated the methanol extract, the alkaloid and the terpenoid fractions of $S$. pilifera for their cytotoxic and antiproliferative activity in vitro (HT-29 cell line), indicating great results [45]. The terpenoid fraction was found to have the best cytotoxic activity compared to the other fractions and as reference compound was used cisplatin. Moreover, they investigated the antiproliferative activity, studying the effects on the activity of caspase- 8 and caspase- 9 , Nuclear factor- $\kappa \mathrm{B}$ (NF- $\mathrm{B}$ ) and Nitric Oxide (NO), reporting that the extract/fractions increased the activity of caspase-8/-9 and decreased NF-кB and subsequently NO level. Of note, they compared their results with previous data of cytotoxic activity in vitro of other Stachys species such as S. acerosa, S. benthamiana, S. floridana, S. lavandulifolia, S. obtusicrena, S. persica, S. pubescens and S. spectabilis. Three isolated compounds from the extract $\left(\mathrm{CH}_{2} \mathrm{Cl}_{2}: \mathrm{MeOH} 1: 1\right)$ of the aerial parts of $S$. aegyptiaca were investigated for the cytotoxic activity in HepG2 cell line, using MTT assay [132]. Precisely, the $\mathrm{IC}_{50}$ values of stachaegyptin D (193), stachysolon monoacetate (178) and stachysolon diacetate (180) were 94.7, 63.4 and $59.5 \mu \mathrm{M}$, respectively, with stachysolone diacetate being the most active. In another study, the cytotoxic effect of the ethanol extract of $S$. riederi var. japonica on UVA-irradiated HDFs was evaluated at different concentrations for $48 \mathrm{~h}$ by MTT assay, showing no or little cytotoxicity [160]. Shakeri et al. (2019) mentioned that the methanol extract of $S$. parviflora demonstrated no cytotoxic activity toward the cancer cell lines, namely A2780, HCT, and B16F10 in all tested concentrations (>100 $\mu \mathrm{g} / \mathrm{mL})$ [64]. Moreover, the genotoxic activity of the extracts from four different plants were investigated by Slapšyte and colleagues (2019) [157]. They reported that all the plant extracts induced DNA damage, using 
the comet assay, whereas the extract of S. officinalis induced the increase of sister chromatid exchange value. The methanol extract of the Lebanese species $S$. ehrenbergii was investigated for its antioxidant and cytotoxic activity [154]. The cytotoxicity was examined by MTT assay where the methanol extract showed the highest cytotoxicity $\left(\mathrm{IC}_{50}=420 \pm 104 \mu \mathrm{g} / \mathrm{mL}\right.$ ) at a concentration of $3000 \mathrm{mg} / \mathrm{mL}$.

\subsection{Polycystic Ovary Syndrome (PCOS)}

In Iran, S. sylvatica is used for the treatment of women with polycystic ovary syndrome (PCOS). A current study carried out by Alizadeh et al. (2020) evaluated the hydroalcoholic extract of this plant in a rat model of PCOS [47]. It was observed that the extract at the dose of $500 \mathrm{mg} / \mathrm{kg}$ increased gonadotropins FSH and LH $(5.95 \pm 0.02 \mathrm{mIU} / \mathrm{mL} ; 6.48 \pm 0.09 \mathrm{mIU} / \mathrm{mL})$ and reduced the level of estrogen $(0.9 \pm 0.07 \mathrm{mIU} / \mathrm{mL}$ ) compared to the PCOS group (FSH level: $1.69 \pm 0.08 \mathrm{mIU} / \mathrm{mL}$; LH level: $6.29 \pm 0.04 \mathrm{mIU} / \mathrm{mL}$; estrogen level: $1.42 \pm 0.05 \mathrm{mIU} / \mathrm{mL}$ ), causing the ratio of $\mathrm{LH} / \mathrm{FSH}$ to be close to 1:1 (6.48/5.59). According to the literature, this ratio LH/FSH is almost 1:1 in normal cases, while in PCOS women is higher e.g., 2:1 or 3:1. They also mentioned that these great results of the extract of S. sylvatica could be correlated to the flavonoid content of the plant. Previous studies showed that flavonoids could decrease the level of estrogen and could also act as GABA receptor agonists, regulating gonadotropins. Given that women with PCOS showed high concentrations of inflammation factors, they assumed that the extract could act as anti-inflammatory and antioxidant agent as flavonoids and iridoids demonstrated antioxidant and anti-inflammatory properties.

\subsection{Anticholinesterase and Anti-Alzheimer's Activity/Neuroprotective Activity}

The aqueous extract from the tubers of $S$. sieboldii ("chorogi") was studied in vivo in mice model for its neuroprotective potential [152]. Specifically, the study examined the effects of chorogi's extract on celebral ischemia and scopolamine-induced memory impairment, using as positive control the extract of Gingko biloba, proving that $S$. sieboldii improves the learning and memory dysfunction correlated with ischemic brain injury. Another work examined the cholinesterase inhibitory activity of S. lavandulifolia extracts and isolated compounds [116]. Specifically, the most active extract against anticholinesterase (AChE) was the $n$-hexane extract with an $\mathrm{IC}_{50}$ value of $13.7 \mu \mathrm{g} / \mathrm{mL}$. However, the dichloromethane extract was the most effective against butyrylcholinesterase $(\mathrm{BChE})\left(\mathrm{IC}_{50}=143.9 \mu \mathrm{g} / \mathrm{mL}\right)$ where its major constituent, stachysolone (177), inhibited the activity of this enzyme with a percentage of inhibition of $50 \%$ at $0.06 \mathrm{mg} / \mathrm{mL}$. Among the studied polar extracts, the methanol extract exhibited a selective inhibitory activity against $\mathrm{AChE}$ with an $\mathrm{IC}_{50}$ value of $211.4 \mu \mathrm{g} / \mathrm{mL}$ and the isolated compounds, arbutin (107) and 5-allosyloxy-aucubin (167), showed a percentage of inhibition of 50 and $23.1 \%$ at $0.06 \mathrm{mg} / \mathrm{mL}$, respectively, against AChE. Notably, the other constituents of this species were inactive at the maximum concentration tested of $0.25 \mathrm{mg} / \mathrm{mL}$. Ferhat et al. (2016) examined the AChE activity of $n$-butanol, the ethyl acetate and the chloroform extracts of the aerial parts of S. guyoniana, demonstrating that the $n$-butanol extract $\left(\mathrm{IC}_{50}=5.78 \pm 0.01 \mu \mathrm{g} / \mathrm{mL}\right)$ was a little less active than the used standard drug against Altzheimer's disease; galantamine ( $\mathrm{IC}_{50}=5.01 \pm 0.10 \mu \mathrm{g} / \mathrm{mL}$ ). Furthermore, they exhibited that this extract inhibited the $\mathrm{BChE}$, having an $\mathrm{IC}_{50}$ value of $39.1 \pm 1.41 \mu \mathrm{g} / \mathrm{mL}$ which was better than the standard $\left(\mathrm{IC}_{50}=39.10 \pm 1.41 \mu \mathrm{g} / \mathrm{mL}\right)$ [155]. Moreover, the anti-Alzheimer's activity of two subspecies of S. cretica (S. cretica subsp. smyrnaea; S. cretica subsp. mersinaea) were evaluated in different works $[81,108]$. In addition, the potential effects of $20 \%$ ethanol extract of S. sieboldii was evaluated against oxidative stress induced by $\mathrm{H}_{2} \mathrm{O}_{2}$ in SK-N-SH cells and memory enhancement in ICR mice [162]. This study showed that the daily intake of the extract (dose: $500 \mathrm{mg} / \mathrm{kg}$ ) through dietary supplementation produced memory enhancing effects in animals. Recently, Ertas and Yener (2020) reported that the acetone extract of $S$. thirkei demonstrated good activity against $\mathrm{AChE}$ and $\mathrm{BChE}$ with a percentage of inhibition of $52.46 \pm 1.26 \%$ and $75.04 \pm 1.91 \%$, respectively [84]. 


\subsection{Anti-tyrosinase Activity}

The anti-tyrosinase activity of the ethanol and methanol Soxhlet apparatus extracts of the aerial parts of $S$. lavandulifolia exhibited the best activity with $\mathrm{IC}_{50}$ values of $33.4 \pm 0.8$ and $42.8 \pm 1.1 \mu \mathrm{g} / \mathrm{mL}$ [116]. They underlay that the specific extracts were characterized by the phenolic compounds, acteoside (118) and arbutin (107), which are recognised as tyosinase inhibitors. Moreover, they evaluated the anti-tyrosinase activity of the isolated iridoids among which monomelittoside (165) and melittoside (166) showed $\mathrm{IC}_{50}$ values of $119.6 \pm 2.2$ and $163.1 \pm 3.1 \mu \mathrm{g} / \mathrm{mL}$ respectively, while 5-allosyloxy-aucubin (167) inhibited the enzyme with a percentage of $22.4 \%$ at a concentration of $200 \mu \mathrm{g} / \mathrm{mL}$. In addition, current works investigated the anti-tyrosinase activity of three subspecies of S. cretica (S. cretica subsp. smyrnaea; S. cretica subsp. mersinaea; S. cretica subsp. vacillans), reporting that the ethyl actetate extract was the most effective in the first two susbspecies ( $2.45 \mathrm{mg}$ KAEs/g; $16 \mathrm{mg}$ KAEs/g, respectively) [81,108]. Though, the methanol extract of $S$. cretica subsp. vacillans had the higher activity against tyrosinase $(314.04 \pm 2.05 \mathrm{mg} \mathrm{KAE} / \mathrm{g}$ extract) [112].

\subsection{Anti-diabetic Activity}

Bahadori et al. (2018) evaluated the anti-diabetic activity of the extracts of S. cretica subsp. smyrnaea [81]. Specifically, the methanol extract demonstrated strong anti-diabetic activity against $\alpha$-amylase (61.4 mg ACEs/g dry plant) and $\alpha$-glucosidase (47.8 mg ACEs/g dry plant), following by ethyl acetate extract. They assumed that the above good properties were attributed to the phenolic constituents of the methanol extract since the anti-glucosidase activity is associated with caffeic acid, trans-cinnamic acid, and vanillin, whereas the amylase inhibitory activity is related to kaempferol and $p$-hydroxybenzoic acid. A year later, the anti-diabetic activity of the extracts of S. cretica subsp. mersinaea was studied, reporting that the ethyl acetate extract had best activity against $\alpha$-amylase (396.50 mgACEs/g), while the methanol extract exerted strong activity against $\alpha$-glucosidase (734 mg ACEs/g) [108]. Furthermore, the $\alpha$-amylase inhibition of the methanol and water extract of $S$. cretica subsp. vacillans was evaluated, with the methanol extract exhibited stronger activity (433.99 $\pm 5.10 \mathrm{mg}$ ACE/g extract) [112]. Currently, Pritsas et al. (2020) studied the anti-diabetic activity in silico of 17 isolated compounds from the cultivated S. iva, mentioning that stachysetin (98) interacted with five out of ten proteins implicated in diabetes [56]. This is the only study reported a pharmacological activity of this rare compound.

\subsection{Antimicrobial Activity}

Regarding the antibacterial activity, the $n$-butanol extract of $S$. guyoniana showed strong activity against Staphylococcus aureus (MIC $=32 \pm 0.90 \mu \mathrm{g} / \mathrm{mL}$ ) and Enterobacter aerogenes (MIC $=32 \pm 0.70 \mu \mathrm{g} / \mathrm{mL}$ ), while it was not active against Pseudomonas aeruginosa and Morganella morganii [155]. The ethyl acetate extract demonstrated the best inhibition against Escherichia coli (MIC $=64 \pm 0.60 \mu \mathrm{g} / \mathrm{mL}$ ), whereas it didn't show any activity against $P$. aeruginosa and M. morganii. Shakeri et al. (2019) reported the antimicrobial activity of the methanol extract of the aerial parts of $S$. parviflora which exerted the highest activity against the Gram-positive bacterium, Bacillus cereus, with a MIC of $0.12 \mathrm{mg} / \mathrm{mL}$ [64]. Furthermore, the antimicrobial activity of extracts of $S$. thirkei against different microorganisms were studied according to inhibition zone diameter and MIC value [84]. The acetone and methanol extract demonstrated good activity against S. aureus, Streptococcus pyogenes and E. coli. Intriguingly, S. thirkeis' extracts were not active against $P$. aeruginosa (Gram-negative bacterium) and Candida albicans (yeast).

\subsection{Hepatoprotective}

The hepatoprotective property of the ethanol extract of S. pilifera was studied in carbon tetrachloride $\left(\mathrm{CCl}_{4}\right)$-induced hepatotoxicity in rats and indicated that this extract could act as hepatoprotective agent [158]. They assumed that this property might be also related to the strong antioxidant activity of the species. Later, Mansourian et al. (2019) exhibited the hepatoprotective and antioxidant activity 
of hydroalcoholic extract of S. pilifera on hepatotoxicity induced by acetaminophen (APAP) in male rats [159]. Precisely, the extract reduced hepatotoxicity by decreasing liver function markers/enzymes, aspartate aminotransferase (AST) and alanine aminotransferase (ALT) and protein carbonyl (PCO) compared to the APAP group. It also diminished the oxidative stress through inhibiting protein oxidation and inducing the activity of glutathione peroxidase (GPX) enzyme. So, they assumed that this great activity was attributed to the antioxidant activity of this plant.

\subsection{Others}

Ruiu et al. (2015) investigated the phytochemical profile of the dichloromethane extract of S. glutinosa and studied the binding affinity to $\mu$ and $\delta$ opioid receptors (MOR and DOR) [107]. The extract showed an interesting binding affinity for MOR (Ki values of $10.3 \mu \mathrm{g} / \mathrm{mL}$ ) and DOR (Ki values of $9.0 \mu \mathrm{g} / \mathrm{mL}$ ), while xanthomicrol (69) demonstrated the strongest opioid binding affinity to both opioid receptors ( $\mathrm{Ki}$ for MOR $=0.83 \mu \mathrm{M}, \mathrm{Ki}$ for DOR $=3.6 \mu \mathrm{M}$ ) with the highest MOR selectivity with a ratio $\mathrm{Ki}(\mathrm{DOR}) / \mathrm{Ki}(\mathrm{MOR})=4.4$. Notably, they reported that the existence of a further hydroxy group at the $3^{\prime}$ position like in sideritoflavone (70) reduced the binding affinity for MOR $(\mathrm{Ki}=18.5 \mu \mathrm{M})$, whereas the replacement of this group with a methoxy moiety, as in 8-methoxycirsilineol (71), eliminated the affinity for MOR $(\mathrm{Ki}>50 \mu \mathrm{M})$. Furthermore, they evaluated the antinociceptive activity of xanthomicrol in an animal model (in mice) of acute pain (tail-flick test). In another study, the $n$-butanol extract of $S$. mialhesii exhibited significant anti-inflammatory activity in vivo, reducing the weight of edema: $52.03 \%$ induced by carrageenan in the rat's paw, whereas indomethacin (dose: $5 \mathrm{mg} / \mathrm{kg}$; decrease $83.36 \%$ ) was used as a reference drug [103]. In the same study, the $n$-butanol extract exerted antinociceptive effect at dose-dependent manner. Ramazanov et al. (2016) evaluated the wound healing activity of the extract of S. hissarica on rats, showing that the extract improved the healing process of linear skin wounds at an oral dose of $10 \mathrm{mg} / \mathrm{kg}$ [67]. Of note, the wound healing activity of the extract was more effective than the known drug methyluracil (2,4-dioxo-6-methyl-1,2,3,4tetrahydropyrimidine), especially in case of alloxan induced diabetic animals. A study carried out by Iannuzzi et al. (2019) studied the antiangiogenic activity in two in vivo models (zebrafish embryos and chick chorioallantoic membrane assays) of the isolated compounds of the leaf extract of S. ocymastrum. The isolated compounds with the best antiangiogenic activity in both assays were $\beta$-hydroxyipolamiide (173) and ipolamiide (174) [123]. Recently, Lee et al. (2020) studied the anti-obesity and anti-dyslipidemic property of the roots powder of S. sieboldii in rats, following a high-fat and high-cholesterol diet (HFC) [161]. This powder demonstrated the anti-adipogenic and lipid-lowering effects through enhancing lipid metabolism.

Taken together all the above pharmacological studies, we could observe that these findings confirmed most of the traditional medicinal uses of Stachys spp. However, the present review unveiled that there are still species pharmacologically uncharted.

\section{Clinical Studies}

Through our literature survey, four clinical studies for the species S. lavandulifolia were revealed. The first clinical study carried out by Rahzani et al. (2013) reported the effects of the aqueous extract of the specific plant (dose; infusion from $3 \mathrm{~g}$ aerial parts of plant, twice daily) on the oxidative stress in 26 healthy humans, underlying that the participants demonstrated a significant reduction in oxidative stress [163]. In parallel, another randomized clinical trial (33 women) examined the effects of S. lavandulifolia and medroxyprogesterone acetate (MPA) in abnormal uterine bleeding (AUB) in PCOS [164]. This study exemplified that the infusion of the aerial parts of wood betony (dose; $5 \mathrm{~g}$ of plant in $100 \mathrm{~mL}$ boiling water; duration 3 months) showed a reduction of AUB, recommending its consumption for the treatment of AUB related to PCOS. They also mentioned that this result might be attributed to the flavonoid content of the plant and mainly to apigenin. In addition, Monji et al. (2018) evaluated on a clinical trial the therapeutic effects of standardized formulation of S. lavandulifolia on primary dysmenorrhea, indicating that the standardized capsules of plant's extract could diminuish 
the menstrual pain, and might be recommended as an auxiliary therapy or an alternative remedy to nonsteroidal antiinflammatory drugs (NSAIDs) with fewer side effects in primary dysmenorrhea [165]. Recently, a double-blind randomized clinical study mentioned the analgesic activity of the herbal tea of S. lavandulifolia (10 g in 200 cc of boiling water) in 50 patients with migraine [166], showing the capability of this herbal tisane to decrease and also improve the pain intensity in these patients. In addition, Ashtiani et al. (2019) considered that the therapeutic properties of this plant associated with its rich phytochemical profile which include iridoids, flavonoids and phenylethanoid glucosides [166].

To sum up, the above clinical studies confirm the ethnomedicinal uses of S. lavandulifolia as a traditional medicine. Although these promising results, more clinical studies should be performed for obtaining data for diverse Stachys spp. As a future prospective, further studies should strengthen the research of bioavailability, dosage, toxicity and potential drug interactions in order to endorse the observed pharmacological activities of these plants.

\section{Toxicity}

S. lavandulifolia is popularly claimed as an abortifacient agent by Iranian women. The effect of its hydroalcoholic extract on fertility was investigated, revealing that the extract had a dose dependent abortifacient activity. Thus, its use during pregnancy may cause abortion and consequently, the plant should be considered as contraindicated or be used with caution [167]. In addition, the nephrotoxicity of the same extract was studied on male Wistar rats and a mild degeneration of renal tubular epithelial cell after one month was observed, while in the second month the histologic lesions were significantly more. However, further studies need to evaluate renal complications of this plant in human [168]. Moreover, the acute and subchronic toxicological evaluation of S. lavandulifolia aqueous extract in rats indicated that the high dose $(2 \mathrm{~g} / \mathrm{kg})$ did not produce any symptoms of toxicity and there was no significant difference in body weights between the control and treatment groups of the animals [169].

\section{Conclusions}

In the present review, we attempted to describe in detail all the current knowledge and research advances of genus Stachys, focusing on pointing the significance of this genus as herbal supplement and medicine.

Taken together with all the analyzed studies in the current review, we categorized the used literature data into four categories according to their general characteristics; ethnobotanical (no of used studies: 48), phytochemical (no of used studies: 91), pharmacological (no of in vitro studies: 22, no of in vivo studies: 8 and 2 in silico study), clinical studies (no of used studies: 4) and reviews (no of used studies: 4). The general characteristics of the analyzed studies in the current review are showed in Table 31.

Table 31. General characteristics of the analyzed studies in the current review.

\begin{tabular}{ccc}
\hline Type of Data & No of Studies * & Years of Publication \\
\hline Ethnobotanical & 48 & since 1914 \\
\hline Phytochemical & 91 & since 1968 \\
\hline \multirow{2}{*}{ Pharmacological } & 22 (in vitro) & \\
\cline { 2 - 2 } & 8 (in vivo) & since 2015 \\
\hline Clinical studies & 2 (in silico) & \\
\hline Reviews & 4 & since 2013 \\
\hline
\end{tabular}

* N.B. It could be found more than one type of data in the same article. 
Several Stachys spp. have been used as traditional herbal medicines for thousands of years. Therefore, accumulating studies have been performed in order to explore the chemical compounds and the pharmacological properties of these species to validate their claimed ethnomedicinal properties. However, the present review data shows that there are still species phytochemically and pharmacologically unexplored. This comprehensive survey could serve as useful tool for scientists searching uncharted and interesting species to study, as well as it could be an informative guide for researchers aimed to identify leads for developing novel drugs. Although many pharmacological studies have demonstrated the great properties of these plants, only the clinical effects of one species have been investigated. As a result, further studies should be performed to validate the clinical efficiency of several Stachys spp. and if there is any potential toxicity. To be mentioned that there are still yet much to be done on the detailed documentation (safety and efficacy data) of genus Stachys in order to be developed an official monograph as a traditional use or well-established use plants.

Author Contributions: Conceptualization and supervision: H.S.; writing-original draft preparation: E.-M.T. \& C.B.; writing - review and editing: all authors. All authors have read and agreed to the published version of the manuscript.

Funding: This research received no external funding.

Conflicts of Interest: The authors declare no conflict of interest.

\section{References}

1. Bhattacharjee, R. Taxonomic studies in Stachys II. A new infragenic classification of Stachys L. [1980]. Notes R. Bot. Gard. Edinbourgh 2008, 38, 65-96.

2. Salmaki, Y.; Heubl, G.; Weigend, M. Towards a new classification of tribe Stachydeae (Lamiaceae): Naming clades using molecular evidence. Bot. J. Linn. Soc. 2019, 190, 345-358. [CrossRef]

3. Tundis, R.; Peruzzi, L.; Menichini, F. Phytochemical and biological studies of Stachys species in relation to chemotaxonomy: A review. Phytochemistry 2014, 102, 7-39. [CrossRef] [PubMed]

4. Koeva-Todorovska, J. The genus Stachys L. and the genus Betonica L. Flora of PR Bulgaria; BAS Publishing House: Sofia, Bulgaria, 1979; Volume 9, pp. 388-416.

5. Marin, P.; Grayer, R.; Grujic-Jovanovic, S.; Kite, G.; Veitch, N. Glycosides of tricetin methyl ethers as chemosystematic markers in Stachys subgenus Betonica. Phytochemistry 2004, 65, 1247-1253. [CrossRef]

6. Strid, A.; Tan, K. Mountain Flora of Greece. II; Edinburgh University Press: Edinburgh, UK, 1991; Volume 2, pp. 97-107.

7. Carnoy, A. Dictionnaire Étymologique des Noms Grecs de Plantes; Bibliothèque du Muséon: Louvain, Paris, $1959 ;$ p. 70.

8. André, J. Lexique des termes de botanique en latin. In Etudes et Commentaires; Librairie, C., Ed.; Klincksieck: Paris, France, 1956; p. 79.

9. Leonis, P. Lexicon Phytologicon; Librairie, C., Ed.; Athens, Greek, 1914; p. 912. (In Greek)

10. Valiakos, E.; Marselos, M.; Sakellaridis, N.; Constantinidis, T.; Skaltsa, H. Ethnopharmacological approach to the herbal medicines of the "Antidotes" in Nikolaos Myrepsos' Dynameron. J. Ethnopharmacol. 2015, 163, 68-82. [CrossRef]

11. Camangi, F.; Stefani, A. Le piante nella magia e nella superstizione: Alcuni esempi di pratiche popolari in Toscana. Riv. Preist. Etnogr. Stor. Nat. 2003, 1, 1-5.

12. Delazar, A.; Delnavazi, M.R.; Nahar, L.; Moghadam, S.B.; Mojarab, M.; Gupta, A.; Williams, A.S.; Rahman, M.M.; Sarker, S.D.; Mojarrab, M. Lavandulifolioside B: A new phenylethanoid glycoside from the aerial parts of Stachys lavandulifolia Vahl. Nat. Prod. Res. 2011, 25, 8-16. [CrossRef]

13. Delnavazi, M.-R.; Saiyarsarai, P.; Jafari-Nodooshan, S.; Khanavi, M.; Tavakoli, S.; Hadavinia, H.; Yassa, N. Cytotoxic flavonoids from the aerial parts of Stachys lavandulifolia Vahl. Pharm. Sci. 2018, 24, 332-339. [CrossRef]

14. Karioti, A.; Bolognesi, L.; Vincieri, F.F.; Bilia, A.R. Analysis of the constituents of aqueous preparations of Stachys recta by HPLC-DAD and HPLC-ESI-MS. J. Pharm. Biomed. Anal. 2010, 53, 15-23. [CrossRef] 
15. Gören, A.C.; Piozzi, F.; Akçiçek, E.; Kılıç, T.; Çarıkçı, S.; Mozioğlu, E.; Setzer, W.N. Essential oil composition of twenty-two Stachys species (mountain tea) and their biological activities. Phytochem. Lett. 2011, 4, 448-453. [CrossRef]

16. Öztürk, M.; Duru, M.E.; Aydoğmuş-Öztürk, F.; Harmandar, M.; Mahlıçlı, M.; Kolak, U.; Ulubelen, A. GC-MS analysis and antimicrobial activity of essential oil of Stachys cretica subsp. smyrnaea. Nat. Prod. Commun. 2009, 4, 109-114. [CrossRef] [PubMed]

17. Khanavi, M.; Sharifzadeh, M.; Hadjiakhoondi, A.; Shafiee, A. Phytochemical investigation and anti-inflammatory activity of aerial parts of Stachys byzanthina C. Koch. J. Ethnopharmacol. 2005, 97, 463-468. [CrossRef] [PubMed]

18. Jahani, R.; Khaledyan, D.; Jahani, A.; Jamshidi, E.; Kamalinejad, M.; Khoramjouy, M.; Faizi, M. Evaluation and comparison of the antidepressant-like activity of Artemisia dracunculus and Stachys lavandulifolia ethanolic extracts: An in vivo study. Res. Pharm. Sci. 2019, 14, 544. [CrossRef] [PubMed]

19. Sadeghi, H.; Mansourian, M.; Kokhdan, E.P.; Salehpour, Z.; Sadati, I.; Abbaszadeh-Goudarzi, K.; Asfaram, A.; Doustimotlagh, A.H. Antioxidant and protective effect of Stachys pilifera Benth against nephrotoxicity induced by cisplatin in rats. J. Food Biochem. 2020, 44, e13190. [CrossRef] [PubMed]

20. Meremeti, A.; Karioti, A.; Skaltsa, H.; Heilmann, J.; Sticher, O. Secondary metabolites from Stachys ionica. Biochem. Syst. Ecol. 2004, 32, 139-151. [CrossRef]

21. Piozzi, F.; Bruno, M. Diterpenoids from roots and aerial parts of the genus Stachys. Rec. Nat. Prod. 2011, 5, 1-11.

22. Gören, A.C. Use of Stachys species (Mountain tea) as herbal tea and food. Rec. Nat. Prod. 2014, 8, 71-82.

23. Kartsev, V.G.; Stepanichenko, N.N.; Auelbekov, S.A. Chemical composition and pharmacological properties of plants of the genus Stachys. Chem. Nat. Compd. 1994, 30, 645-654. [CrossRef]

24. Plant List. 2013. Available online: http://www.theplantlist.org/tpl1.1/record/kew-195205 (accessed on 4 August 2020).

25. The Euro + Med Plantbase. The Information Resource for Euro-Mediterranean Plant Diversity. Available online: http://ww2.bgbm.org/EuroPlusMed/query.asp (accessed on 4 August 2020).

26. International Plant Name Index (IPNI). Available online: https://www.ipni.org/n/459647-1 (accessed on 4 August 2020).

27. Venditti, A.; Frezza, C.; Celona, D.; Bianco, A.; Serafini, M.; Cianfaglione, K.; Fiorini, D.; Ferraro, S.; Maggi, F.; Lizzi, A.R.; et al. Polar constituents, protection against reactive oxygen species, and nutritional value of Chinese artichoke (Stachys affinis Bunge). Food Chem. 2017, 221, 473-481. [CrossRef]

28. Guo, H.; Saravanakumar, K.; Wang, M.-H. Total phenolic, flavonoid contents and free radical scavenging capacity of extracts from tubers of Stachys affinis. Biocatal. Agric. Biotechnol. 2018, 15, 235-239. [CrossRef]

29. Lee, J.W.; Wu, W.; Lim, S.Y. Effect of extracts from Stachys sieboldii Miq. on cellular reactive oxygen species and glutathione production and genomic DNA oxidation. Asian Pac. J. Trop. Biomed. 2018, 8, 485. [CrossRef]

30. Huang, W.; Gao, X.; Zhang, Y.; Jin, C.; Wang, X. The complete chloroplast genome sequence of Stachys sieboldii Miquel. (Labiatae), a kind of vegetable crop and Chinese medicinal material plant. Mitochondrial DNA Part B 2020, 5, 1832-1833. [CrossRef]

31. Asghari, G.; Akbari, M.; Asadi-Samani, M. Phytochemical analysis of some plants from Lamiaceae family frequently used in folk medicine in Aligudarz region of Lorestan province. Marmara Pharm. J. 2017, 21, 506. [CrossRef]

32. Lotfipour, F.; Nazemiyeh, H.; Fathi-Azad, F.; Garaei, N.; Arami, S.; Talat, S.; Sadegpour, F.; Hasanpour, R. Evaluation of antibacterial activities of some medicinal plants from North-West Iran. Iran. J. Basic Med. Sci. 2008, 11, 80-85.

33. Asnaashari, S.; Delazar, A.; Alipour, S.; Nahar, L.; Williams, A.; Pasdaran, A.; Mojarab, M.; Azad, F.; Sarker, S.D. Chemical composition, free-radical-scavenging and insecticidal activities of the aerial parts of Stachys byzantina. Arch. Biol. Sci. 2010, 62, 653-662. [CrossRef]

34. Naghibi, F.; Mosaddegh, M.; Motamed, M.M.; Ghorbani, A. Labiatae family in folk medicine in Iran: From ethnobotany to pharmacology. Iran. J. Pharm. Res. 2005, 2, 63-79.

35. Aminfar, P.; Abtahi, M.; Parastar, H. Gas chromatographic fingerprint analysis of secondary metabolites of Stachys lanata (Stachys byzantine C. Koch) combined with antioxidant activity modelling using multivariate chemometric methods. J. Chromatogr. A 2019, 1602, 432-440. [CrossRef] 
36. Maleki, N.; Garjani, A.; Nazemiyeh, H.; Nilfouroushan, N.; Sadat, A.E.; Allameh, Z.; Hasannia, N. Potent anti-inflammatory activities of hydroalcoholic extract from aerial parts of Stachys inflata on rats. J. Ethnopharmacol. 2001, 75, 213-218. [CrossRef]

37. Lazarević, J.; Palić, R.; Radulović, N.S.; Ristic, N.; Stojanovic, G. Chemical composition and screening of the antimicrobial and anti-oxidative activity of extracts of Stachys species. J. Serb. Chem. Soc. 2010, 75, 1347-1359. [CrossRef]

38. Amin, G. Popular medicinal plants of Iran, Iranian Research Institute of medicinal plants. Tehran 1991, 80, 1-66. (In Persian)

39. Rabbani, M.; Sajjadi, S.E.; Zarei, H. Anxiolytic effects of Stachys lavandulifolia Vahl on the elevated plus-maze model of anxiety in mice. J. Ethnopharmacol. 2003, 89, 271-276. [CrossRef] [PubMed]

40. Rabbani, M.; Sajjadi, S.-E.; Karimi-Firozjai, M.; Ghannadian, M. Bioactivity guided isolation of apigenin from Stachys lavandulifolia Vahl. in mice with anxiolytic effects. J. Herbmed Pharmacol. 2018, 7, 74-78. [CrossRef]

41. Hajhashemi, V.; Ghannadi, A.; Sedighifar, S. Analgesic and anti-inflammatory properties of the hydroalcoholic, polyphenolic and boiled extracts of Stachys lavandulifolia. Res. Pharm. Sci. 2007, 2, 92-98.

42. Arabsalehi, F.; Rahimmalek, M.; Ehtemam, M.H. Phytochemical and morphological variation of Stachys lavandulifolia Vahl. populations as affected by genotype $\times$ year interaction. Ind. Crop. Prod. 2018, 112, 342-352. [CrossRef]

43. Veisi, H.; Kazemi, S.; Mohammadi, P.; Safarimehr, P.; Hemmati, S. Catalytic reduction of 4-nitrophenol over Ag nanoparticles immobilized on Stachys lavandulifolia extract-modified multi walled carbon nanotubes. Polyhedron 2019, 157, 232-240. [CrossRef]

44. Fooladvand, Z.; Fazeli-nasab, B. Antibacterial activities of Stachys lavandulifolia Vahl. extract against eight bacteria. J. Herb. Drugs 2014, 5, 13-18.

45. Kokhdan, E.P.; Sadeghi, H.; Ghafoori, H.; Sadeghi, H.; Danaei, N.; Javadian, H.; Aghamaali, M.R. Cytotoxic effect of methanolic extract, alkaloid and terpenoid fractions of Stachys pilifera against HT-29 cell line. Res. Pharm. Sci. 2018, 13, 404-412. [CrossRef]

46. Maleki, F.; Valilou, M.M.S. Poulk plant (Stachys schtschegleevii) and its antibacterial specifications. Asian J. Res. Bot. 2019, 2, 1-13.

47. Alizadeh, F.; Ramezani, M.; Piravar, Z. Effects of Stachys sylvatica hydroalcoholic extract on the ovary and hypophysis-gonadal axis in a rat with polycystic ovary syndrome. Middle East Fertil. Soc. J. 2020, 25, 1-7. [CrossRef]

48. Polat, R.; Cakilcioglu, U.; Kaltalioğlu, K.; Ulusan, M.D.; Türkmen, Z. An ethnobotanical study on medicinal plants in Espiye and its surrounding (Giresun-Turkey). J. Ethnopharmacol. 2015, 163, 1-11. [CrossRef]

49. Altundag, E.; Öztürk, M. Ethnomedicinal studies on the plant resources of east Anatolia, Turkey. Procedia Soc. Behav. Sci. 2011, 19, 756-777. [CrossRef]

50. Mükemre, M.; Behçet, L.; Çakılcıŏlu, U.; Cakilcioglu, U. Ethnobotanical study on medicinal plants in villages of Çatak (Van-Turkey). J. Ethnopharmacol. 2015, 166, 361-374. [CrossRef]

51. Lucchetti, L.; Zitti, S.; Taffetani, F. Ethnobotanical uses in the Ancona district (Marche region, Central Italy). J. Ethnobiol. Ethnomed. 2019, 15, 1-33. [CrossRef] [PubMed]

52. Venditti, A.; Bianco, A.; Quassinti, L.; Bramucci, M.; Lupidi, G.; Damiano, S.; Papa, F.; Vittori, S.; Bini, L.M.; Giuliani, C.; et al. Phytochemical analysis, biological activity, and secretory structures of Stachys annua (L.) L. subsp. annua (Lamiaceae) from Central Italy. Chem. Biodivers. 2015, 12, 1172-1183. [PubMed]

53. Cornara, L.; La Rocca, A.; Marsili, S.; Mariotti, M.G. Traditional uses of plants in the Eastern Riviera (Liguria, Italy). J. Ethnopharmacol. 2009, 125, 16-30. [CrossRef]

54. Cornara, L.; La Rocca, A.; Terrizzano, L.; Dente, F.; Mariotti, M.G. Ethnobotanical and phytomedical knowledge in the North-Western Ligurian Alps. J. Ethnopharmacol. 2014, 155, 463-484. [CrossRef]

55. Mulas, M. Traditional uses of labiatae in the mediterranean area. Acta Hortic. 2006, 723, 25-32. [CrossRef]

56. Pritsas, A.; Tomou, E.-M.; Tsitsigianni, E.; Papaemmanouil, C.D.; Diamantis, D.A.; Chatzopoulou, P.; Tzakos, A.G.; Skaltsa, H. Valorisation of stachysetin from cultivated Stachys iva Griseb. as anti-diabetic agent: A multi-spectroscopic and molecular docking approach. J. Biomol. Struct. Dyn. 2020, 1-15. [CrossRef]

57. Fazio, C.; Passannanti, S.; Paternostro, M.; Arnold, N. Diterpenoids from Stachys mucronata. Planta Med. 1994, 60, 499. [CrossRef]

58. Łuczaj, Ł.; Svanberg, I.; Köhler, P. Marsh woundwort, Stachys palustris L. (Lamiaceae): An overlooked food plant. Genet. Resour. Crop. Evol. 2011, 58, 783-793. [CrossRef] 
59. Monigatti, M.; Bussmann, R.W.; Weckerle, C.S. Medicinal plant use in two Andean communities located at different altitudes in the Bolívar Province, Peru. J. Ethnopharmacol. 2013, 145, 450-464. [CrossRef] [PubMed]

60. Duarte, M.C.T.; Figueira, G.M.; Sartoratto, A.; Rehder, V.L.G.; Delarmelina, C. Anti-Candida activity of Brazilian medicinal plants. J. Ethnopharmacol. 2005, 97, 305-311. [CrossRef] [PubMed]

61. Gruenwald, J.; Brendleer, T.; Jaenicke, T. PDR for Herbal Medicines; Medical Economics Company: Montvale, NJ, USA, 2000; p. 832.

62. Rustaiyan, A.; Masoudi, S.; Ameri, N.; Samiee, K.; Monfared, A. Volatile constituents of Ballota aucheri Boiss., Stachys benthamiana Boiss. and Perovskia abrotanoides Karel. Growing wild in Iran. J. Essent. Oil Res. 2006, 18, 218-221. [CrossRef]

63. Ahmad, V.U.; Arshad, S.; Bader, S.; Iqbal, S.; Khan, A.; Khan, S.S.; Hussain, J.; Tareen, R.B.; Ahmed, A. New terpenoids from Stachys parviflora Benth. Magn. Reson. Chem. 2008, 46, 986-989. [CrossRef]

64. Shakeri, A.; D’Urso, G.; Taghizadeh, S.F.; Piacente, S.; Norouzi, S.; Soheili, V.; Asili, J.; Salarbashi, D. LC-ESI/LTQOrbitrap/MS/MS and GC-MS profiling of Stachys parviflora L. and evaluation of its biological activities. J. Pharm. Biomed. Anal. 2019, 168, 209-216. [CrossRef] [PubMed]

65. Kepekçi, R.A.; Polat, S.; Çoşkun, G.; Celik, A.; Bozkurt, A.S.; Yumrutaş, Ö.; Pehlivan, M. Preliminary characterization of phenolic acid composition and hepatoprotective effect of Stachys pumila. J. Food Biochem. 2016, 41, 12286. [CrossRef]

66. Kumar, D.; Bhat, Z.A. Apigenin 7-glucoside from Stachys tibetica Vatke and its anxiolytic effect in rats. Phytomedicine 2014, 21, 1010-1014. [CrossRef]

67. Ramazanov, N.S.; Bobayev, I.D.; Yusupova, U.Y.; Aliyeva, N.K.; Egamova, F.R.; Yuldasheva, N.Y.; Syrov, V.N. Phytoecdysteroids-containing extract from Stachys hissarica plant and its wound-healing activity. Nat. Prod. Res. 2016, 31, 593-597. [CrossRef]

68. El-Ansari, M.A.; Abdalla, M.F.; Saleh, N.A.M.; Barron, D.; Le Quere, J.L. Flavonoid constituents of Stachys aegyptiaca. Phytochemistry 1991, 30, 1169-1173. [CrossRef]

69. El-Ansari, M.A.; Nawwar, M.A.; Saleh, N.A.M. Stachysetin, a diapigenin-7-glucoside-p, p'-dihydroxy-truxinate from Stachys aegyptiaca. Phytochemistry 1995, 40, 1543-1548. [CrossRef]

70. El-Desoky, S.K.; Hawas, W.U.; Sharaf, M. A new flavone glycoside from Stachys aegyptiaca. Chem. Nat. Compd. 2007, 43, 542-543. [CrossRef]

71. Sharaf, M. Isoscutellarein 8-O-(6"-trans-p-coumaroyl)- $\beta$-D-glucoside from Stachys aegyptiaca. Fitoterapia 1998, 69, 355-357.

72. Komissarenko, N.F.; Sheremet, I.P.; Derkach, A.I.; Pakaln, D.A. Stachyflaside from Stachys inflata and St. atherocalyx. Chem. Nat. Compd. 1976, 12, 88. [CrossRef]

73. Komissarenko, N.F.; Derkach, A.I.; Sheremet, I.P.; Kovalev, I.P.; Gordienko, V.G.; Pakaln, D.A. Flavonoids of Stachys inflata. Chem. Nat. Compd. 1978, 14, 445-446. [CrossRef]

74. Nazemiyeh, H.; Shoeb, M.; Movahhedin, N.; Kumarasamy, Y.; Talebpour, A.; Delazar, A.; Lutfun, N.; Sarker, S. Phenolic compounds and their glycosides from Stachys schtscheglevii (Lamiaceae). Biochem. Syst. Ecol. 2006, 34, 721-723. [CrossRef]

75. Tomás-Barberán, F.A.; Gil, M.I.; Ferreres, F.; Tomás-Lorente, F. Flavonoid p-coumaroylglucosides and 8-hydroxyflavone allosylglucosides in some Labiatae. Phytochemistry 1992, 31, 3097-3102. [CrossRef]

76. Lakhal, H.; Boudiar, T.; Kabouche, A.; Laggoune, S.; Kabouche, Z.; Topçu, G. Antioxidant activity and flavonoids of Stachys ocymastrum. Chem. Nat. Compd. 2011, 46, 964-965. [CrossRef]

77. Skaltsa, H.; Bermejo, P.; Lazari, D.; Silván, A.M.; Skaltsounis, A.-L.; Sanz, A.; Abad, M.J. Inhibition of prostaglandin E2 and leukotriene $\mathrm{C} 4$ in mouse peritoneal macrophages and thromboxane B2 production in human platelets by flavonoids from Stachys chrysantha and Stachys candida. Biol. Pharm. Bull. 2000, 23, 47-53. [CrossRef]

78. Michailidou, A.-M. Phytochemical study of Stachys candida Bory \& Chaub. Master's Thesis, National and Kapodistrian University of Athens, Athens, Greece, 2018.

79. Serrilli, A.; Ramunno, A.; Piccioni, F.; Serafini, M.; Ballero, M. Flavonoids and iridoids from Stachys corsica. Nat. Prod. Res. 2005, 19, 561-565. [CrossRef]

80. Demirtaş, I.; Gecibesler, I.H.; Yaglioglu, A.S.; Yaglioglu, A.S. Antiproliferative activities of isolated flavone glycosides and fatty acids from Stachys byzantina. Phytochem. Lett. 2013, 6, 209-214. [CrossRef] 
81. Bahadori, M.B.; Kirkan, B.; Sarikurkcu, C. Phenolic ingredients and therapeutic potential of Stachys cretica subsp. smyrnaea for the management of oxidative stress, Alzheimer's disease, hyperglycemia, and melasma. Ind. Crop. Prod. 2019, 127, 82-87. [CrossRef]

82. Murata, T.; Endo, Y.; Miyase, T.; Yoshizaki, F. Iridoid glycoside constituents of Stachys lanata. J. Nat. Prod. 2008, 71, 1768-1770. [CrossRef]

83. Derkach, A.I.; Komissarenko, N.F.; Gordienko, V.G.; Sheremet, I.P.; Kovalev, I.P.; Pakaln, D.A. Flavonoids of Stachys spectabilis. Chem. Nat. Compd. 1980, 16, 128-130. [CrossRef]

84. Ertas, A.; Yener, I. A comprehensive study on chemical and biological profiles of three herbal teas in Anatolia; rosmarinic and chlorogenic acids. S. Afr. J. Bot. 2020, 130, 274-281. [CrossRef]

85. Elfalleh, W.; Kirkan, B.; Sarikurkcu, C. Antioxidant potential and phenolic composition of extracts from Stachys tmolea: An endemic plant from Turkey. Ind. Crop. Prod. 2019, 127, 212-216. [CrossRef]

86. Venditti, A.; Bianco, A.; Nicoletti, M.; Quassinti, L.; Bramucci, M.; Lupidi, G.; Vitali, L.A.; Papa, F.; Vittori, S.; Petrelli, D.; et al. Characterization of secondary metabolites, biological activity and glandular trichomes of Stachys tymphaea hausskn. from the Monti Sibillini National Park (Central Apennines, Italy). Chem. Biodivers. 2014, 11, 245-261. [CrossRef] [PubMed]

87. Sen, A.; Göğer, F.; Dogan, A.; Bitis, L. Two acylated isoscutellarein glucosides with anti-inflammatory and antioxidant activities isolated from endemic Stachys subnuda Montbret \& Aucher ex Benth. Acta Chim. Slov. 2019, 66, 831-838. [CrossRef]

88. Kostyuchenko, O.I.; Komissarenko, N.F.; Zinchenko, T.V.; Derkach, A.I. Diacetylstachyflaside from Stachys atherocalyx. Khim. Prir. Soedin. 1981, 3, 389-390.

89. Kostyuchenko, O.I.; Komissarenko, N.F.; Kovalev, I.P.; Derkach, A.I.; Gordienko, V.G. Acetylspectabiflaside from Stachys atherocalyx. Chem. Nat. Compd. 1982, 18, 170-172. [CrossRef]

90. Kostyuchenko, O.I.; Komissarenko, N.F.; Zinchenko, T.V.; Derkach, A.I.; Gordienko, V.G. Diacetylisostachyflaside and acetylisostachyflaside from Stachys atherocalyx. Chem. Nat. Compd. 1982, 18, 235-236. [CrossRef]

91. Lenherr, A.; Lahloub, M.F.; Sticher, O. Three flavonoid glucosides containing acetylated allose from Stachys recta. Phytochemistry 1984, 23, 2343-2345. [CrossRef]

92. Lenherr, A.; Meier, B.; Sticher, O. Modern HPLC as a tool for chemotaxonomical investigations: Iridoid glucosides and acetylated flavonoids in the group of Stachys recta. Planta Med. 1984, 50, 403-409. [CrossRef] [PubMed]

93. Sheremet, I.P.; Komissarenko, N.F. Flavonoid glucosides of Stachys annua. Khim. Prir. Soedin 1971, 5, 583.

94. Movsumov, I.S.; Yusifova, D.Y.; Suleimanov, T.A.; Mahiou-Leddet, V.; Herbette, G.; Baghdikian, B.; Ollivier, E.; Garayev, E.E.; Garayev, E.A. Biologically active compounds from chamaenerion angustifolium and Stachys annua growing in Azerbaidzhan. Chem. Nat. Compd. 2016, 52, 324-325. [CrossRef]

95. Movsumov, I.S.; Garayev, E.A.; Baghdikian, B.; Mabrouki, F.; Herbette, G.; Ollivier, E.; Suleimanov, T.A.; Garayev, E.E. Flavonoids from Stachys annua growing in Azerbaijan. Chem. Nat. Compd. 2018, 54, 261-262. [CrossRef]

96. Delazar, A.; Celik, S.; Göktürk, R.S.; Unal, O.; Nahar, L.; Sarker, S.D. Two acylated flavonoid glycosides from Stachys bombycina, and their free radical scavenging activity. Die Pharm. 2005, 60, 878-880. [CrossRef]

97. Zinchenko, T.V. Flavonoids of Stachys neglecta. Farm. Zhurnal 1969, 24, 28804.

98. Kotsos, M.; Aligiannis, N.; Mitaku, S.; Skaltsounis, A.-L.; Charvala, C. Chemistry of plants from Crete: Stachyspinoside, a new flavonoid glycoside and iridoids from Stachys spinosa. Nat. Prod. Lett. 2001, 15, 377-386. [CrossRef]

99. Kotsos, M.P.; Aligiannis, N.; Mitakou, S. A new flavonoid diglycoside and triterpenoids from Stachys spinosa L. (Lamiaceae). Biochem. Syst. Ecol. 2007, 35, 381-385. [CrossRef]

100. Afouxenidi, A.; Milošević-Ifantis, T.; Skaltsa, H. Secondary metabolites from Stachys tetragona Boiss. \& Heldr. ex Boiss. and their chemotaxonomic significance. Biochem. Syst. Ecol. 2018, 81, 83-85. [CrossRef]

101. Lenherr, A.; Mabry, T.J. Acetylated allose-containing flavonoid glucosides from Stachys anisochila. Phytochemistry 1987, 26, 1185-1188. [CrossRef]

102. Skaltsa, H.; Georgakopoulos, P.; Lazari, D.; Karioti, A.; Heilmann, J.; Sticher, O.; Constantinidis, T. Flavonoids as chemotaxonomic markers in the polymorfic Stachys swainsonii (Lamiaceae). Biochem. Syst. Ecol. 2007, 35, 317-320. [CrossRef] 
103. Laggoune, S.; Zeghib, A.; Kabouche, A.; Kabouche, Z.; Maklad, Y.A.; Leon, F.; Brouard, I.; Bermejo, J.; Calliste, C.A.; Duroux, J.L. Components and antioxidant, anti-inflammatory, anti-ulcer and antinociceptive activities of the endemic species Stachys mialhesii de Noé. Arab. J. Chem. 2016, 9, S191-S197. [CrossRef]

104. Zinchenko, T.V. Phenolic compounds of Stachys palustris. Chem. Nat. Compd. 1973, 6, 261-262. [CrossRef]

105. Litvinenko, V.I.; Aronova, B.N. Phenolic compounds of Betonica foliosa. Chem. Nat. Compd. 1968, 4, 270. [CrossRef]

106. Hegazy, M.-E.F.; Hamed, A.R.; El-Kashoury, E.-S.A.; Shaheen, A.M.; Tawfik, W.A.; Paré, P.W.; Abdel-Sattar, E.A. Stachaegyptin A-C: Neo clerodane diterpenes from Stachys aegyptiaca. Phytochem. Lett. 2017, 21, 151-156. [CrossRef]

107. Ruiu, S.; Anzani, N.; Orruù, A.; Floris, C.; Caboni, P.; Alcaro, S.; Maccioni, E.; Distinto, S.; Cottiglia, F. Methoxyflavones from Stachys glutinosa with binding affinity to opioid receptors: In Silico, in vitro, and in vivo studies. J. Nat. Prod. 2015, 78, 69-76. [CrossRef]

108. Bahadori, M.B.; Kirkan, B.; Sarikurkcu, C.; Ceylan, O. Metabolite profiling and health benefits of Stachys cretica subsp. mersinaea as a medicinal food. Ind. Crop. Prod. 2019, 131, 85-89. [CrossRef]

109. Venditti, A.; Serrilli, A.; Di Cecco, M.; Ciaschetti, G.; Andrisano, T.; Bianco, A. Phytochemical composition of polar fraction of Stachys germanica L. subsp. salviifolia (Ten.) Gams, a typical plant of Majella National Park. Nat. Prod. Res. 2013, 27, 190-193. [CrossRef]

110. Litvinenko, V.I. Some Questions of the Chemistry and Taxonomy of the Labiatae Family, the Plant Resources of the Ukraine, Their Isolation and Rational Use; Naukova Dumka: Kiev, Ukraine, 1973; p. 128.

111. Šliumpaitè, I.; Venskutonis, P.; Murkovic, M.; Ragažinskienė, O. Antioxidant properties and phenolic composition of wood betony (Betonica officinalis L., syn. Stachys officinalis L.). Ind. Crop. Prod. 2013, 50, 715-722. [CrossRef]

112. Kirkan, B. Antioxidant potential, enzyme inhibition activity, and phenolic profile of extracts from Stachys cretica subsp. vacillans. Ind. Crop. Prod. 2019, 140, 111639. [CrossRef]

113. Nishimura, H.; Sasaki, H.; Inagaki, N.; Chin, M.; Mitsuhashi, H.; Masao, C.; Chen, Z. Nine phenethyl alcohol glycosides from Stachys sieboldii. Phytochemistry 1991, 30, 965-969. [CrossRef]

114. Ikeda, T.; Miyase, T.; Ueno, A. Phenylethanoid glycosides from Stachys riederi. Nat. Med. 1994, 48, 32-38. (In Japanese)

115. Başaran, A.A.; Calis, I.; Anklin, C.; Nishibe, S.; Sticher, O. Lavandulifolioside: A new phenylpropanoid glycoside from Stachys lavandulifolia. Helv. Chim. Acta 1988, 71, 1483-1490. [CrossRef]

116. Tundis, R.; Bonesi, M.; Pugliese, A.; Nadjafi, F.; Menichini, F.; Loizzo, M.R. Tyrosinase, Acetyl- and Butyryl-cholinesterase inhibitory activity of Stachys lavandulifolia Vahl (Lamiaceae) and its major constituents. Rec. Nat. Prod. 2015, 9, 81-93.

117. Çalis, I.; Basaran, A.; Saracoglu, C.; Sticher, O. Iridoid and phenylopropanoid glycosides from Stachys macrantha. Phytochemistry 1992, 31, 167-169. [CrossRef]

118. Miyase, T.; Yamamoto, R.; Ueno, A. Phenylethanoid glycosides from Stachys officinalis. Phytochemistry 1996, 43, 475-479. [CrossRef]

119. Venditti, A.; Bianco, A.; Nicoletti, M.; Quassinti, L.; Bramucci, M.; Lupidi, G.; Vitali, L.A.; Petrelli, D.; Papa, F.; Vittori, S.; et al. Phytochemical analysis, biological evaluation and 2 micromorphological study of Stachys alopecuros (L.) Benth. subsp. divulsa (Ten.) Grande endemic to central Apennines, Italy. Fitoterapia 2013, 90, 94-103.

120. Ahmad, V.U.; Arshad, S.; Bader, S.; Ahmed, A.; Iqbal, S.; Tareen, R.B. New phenethyl alcohol glycosides from Stachys parviflora. J. Asian Nat. Prod. Res. 2006, 8, 105-111. [CrossRef]

121. Komissarenko, N.F.; Derkach, A.I.; Sheremet, I.P.; Pakaln, D.A. Iridoids of Stachys inflata and St. iberica. Khim. Prir. Soedin. 1979, 1, 99-100.

122. Serrilli, A.M.; Ramunno, A.; Piccioni, F.; Serafini, M.; Ballero, M.; Bianco, A. Monoterpenoids from Stachys glutinosa L. Nat. Prod. Res. 2006, 20, 648-652. [CrossRef] [PubMed]

123. Iannuzzi, A.M.; Camero, C.M.; D’ambola, M.; D’angelo, V.; Amira, S.; Bader, A.; Braca, A.; De Tommasi, N.; Germanò, M.P. Antiangiogenic Iridoids from Stachys ocymastrum and Premna resinosa. Planta Med. 2019, 85, 1034-1039. [CrossRef] [PubMed]

124. Háznagy-Radnai, E.; Czigle, S.; Janicsák, G.; Máthé, I. Iridoids of Stachys species growing in Hungary. J. Planar Chromatogr. Mod. TLC 2006, 19, 187-190. [CrossRef] 
125. Derkach, A.I.; Komissarenko, N.F.; Pakaln, D.A. Iridoids from some Stachys L. species. Rast Nye Resur. 1987, 23, 92-95.

126. Litvinenko, V.I.; Aronova, B.N. Iridoids of Betonica foliosa. Chem. Nat. Compd. 1968, 4, 269-270. [CrossRef]

127. Jeker, M.; Sticher, O.; Calis, I.; Rüedi, P. Allobetonicoside and 6-O-Acetylmioporoside: Two new iridoid glycosides from Betonica officinalis L. Helv. Chim. Acta 1989, 72, 1787-1791. [CrossRef]

128. Zinchenko, T.V. Stachys and Betonica iridoids. Farm. Zhurnal 1972, 27, 86-87.

129. Muñoz, O.; Peña, R.C.; Montenegro, G. Iridoids from Stachys grandidentata. Z. Nat. C 2001, 56, $902-903$. [CrossRef]

130. Melek, F.R.; Radwan, A.S.; El-Ansari, M.A.; El-Gindi, O.D.; Hilal, S.H.; Genenah, A.A. Diterpenes from Stachys aegyptiaca. Fitoterapia 1992, 63, 276.

131. Mohamed, A.E.-H.H.; Mohamed, N.S. A new trans-neo clerodane diterpene from Stachys aegyptiaca. Nat. Prod. Res. 2013, 28, 30-34. [CrossRef]

132. Mohamed, T.A.; ElShamy, A.I.; Hamed, A.R.; Shams, K.A.; Hegazy, M.-E.F. Cytotoxic neo-clerodane diterpenes from Stachys aegyptiaca. Phytochem. Lett. 2018, 28, 32-36. [CrossRef]

133. Hussien, T.A.; Mahmoud, A.A.; Mohamed, N.S.E.-D.; Shahat, A.A.; El-Seedi, H.R.; Hegazy, M.-E.F. New rare ent-clerodane diterpene peroxides from Egyptian Mountain Tea (Qourtom) and its chemosystem as herbal remedies and phytonutrients agents. Molecules 2020, 25, 2172. [CrossRef] [PubMed]

134. Derkach, A.I. Biologically active substances of some species of the genus Stachys L. of the flora of the Ukraine. Rastit. Nye Resur. 1998, 34, 57-61.

135. Piozzi, F.; Savona, G.; Hanson, J.R. Kaurenoid diterpenes from Stachys lanata. Phytochemistry 1980, 19, 1237-1238. [CrossRef]

136. Orgiyan, T.M.; Popa, D.P. Diterpenoids from Stachys annua. Khim. Prir. Soedin. 1969, 5, 5-6. [CrossRef]

137. Popa, D.P.; Orgiyan, T.M. The stereochemistry of stachysolone. Khim. Prir. Soedin. 1972, 8, 717-719. [CrossRef]

138. Popa, D.P.; Orgiyan, T.M. Minor diterpenoids of Stachys annua. Chem. Nat. Compd. 1974, 10, 410. [CrossRef]

139. Piozzi, F.; Paternostro, M.; Servettaz, O.; Arnold, N. Occurrence of (+)-6-desoxyandalusol in Stachys ionica and Stachys distans. Biochem. Syst. Ecol. 2002, 30, 887-889. [CrossRef]

140. Adinolfi, M.; Barone, G.; Lanzetta, R.; Laonigro, G.; Mangoni, L.; Parrilli, M. Diterpenes from Stachys recta. J. Nat. Prod. 1984, 47, 541-543. [CrossRef]

141. Fazio, C.; Paternostro, M.P.; Passannanti, S.; Piozzi, F. Further neo-clerodane diterpenoids from Stachys rosea. Phytochemistry 1994, 37, 501-503. [CrossRef]

142. Popa, D.P.; Pasechnik, G.S. Structure of stachysic acid-A new diterpenoid of the kaurane series. Chem. Nat. Compd. 1974, 10, 454-457. [CrossRef]

143. Bankova, V.; Koeva-Todorovska, J.; Stambolijska, T.; Ignatova-Groceva, M.-D.; Todorova, D.; Popov, S. Polyphenols in Stachys and Betonica species (Lamiaceae). Z. Nat. C 1999, 54, 876-880. [CrossRef]

144. Paternostro, M.P.; Maggio, A.M.; Piozzi, F.; Servettaz, O. Lavdane diterpenes from Stachys plumose. J. Nat. Prod. 2000, 63, 1166-1167. [CrossRef] [PubMed]

145. Miyase, T.; Yamamoto, R.; Ueno, A. Betonicosides A-D and betonicolide, diterpenoids from the roots of Stachys officinalis. Chem. Pharm. Bull. 1996, 44, 1610-1613. [CrossRef]

146. Ross, S.A.; Zinchenko, T.V. Triterpenoids and steroids from Stachys palustris. Farm. Zhurnal 1975, 30, 91-92. [PubMed]

147. Yamamoto, R.; Miyase, T.; Ueno, A. Stachyssaponins I-VIII, new oleanane-type triterpene saponins from Stachys riederi CHAMISSO. Chem. Pharm. Bull. 1994, 42, 1291-1296. [CrossRef]

148. Takeda, Y.; Zhang, H.-J.; Masuda, T.; Honda, G.; Otsuka, H.; Sezik, E.; Yesilada, E.; Sun, H. Megastigmane glucosides from Stachys byzantina. Phytochemistry 1997, 44, 1335-1337. [CrossRef]

149. Rodríguez-García, C.; Sánchez-Quesada, C.; Toledo, E.; Delgado-Rodríguez, M.; Gaforio, J.J. Naturally lignan-rich foods: A dietary tool for health promotion? Molecules 2019, 24, 917. [CrossRef]

150. Sørensen, M.; Penn, M.; El Mowafi, A.; Storebakken, T.; Chunfang, C.; Øverland, M.; Krogdahl, Å. Effect of stachyose, raffinose and soya-saponins supplementation on nutrient digestibility, digestive enzymes, gut morphology and growth performance in Atlantic salmon (Salmo salar, L.). Aquaculture 2011, 314, 145-152. [CrossRef] 
151. Yin, J.; Yang, G.; Wang, S.; Chen, Y. Purification and determination of stachyose in Chinese artichoke (Stachys sieboldii Miq.) by high-performance liquid chromatography with evaporative light scattering detection. Talanta 2006, 70, 208-212. [CrossRef]

152. Harada, S.; Tsujita, T.; Ono, A.; Miyagi, K.; Mori, T.; Tokuyama, S. Stachys sieboldii (Labiatae, Chorogi) protects against learning and memory dysfunction associated with ischemic brain injury. J. Nutr. Sci. Vitaminol. 2015, 61, 167-174. [CrossRef] [PubMed]

153. Sarikurkcu, C.; Kocak, M.S.; Uren, M.C.; Calapoglu, M.; Tepe, A.S. Potential sources for the management global health problems and oxidative stress: Stachys byzantina and S. iberica subsp. iberica var. densipilosa. Eur. J. Integr. Med. 2016, 8, 631-637. [CrossRef]

154. Abi-Rizk, A.; El Rayess, Y.; Iriti, M.; Tabet, E.; Mezher, R.; El Beyrouthy, M. Chemical composition, antitumor and antioxidant effects of four lebanese plants extracts on human pulmonary adenocarcinoma. Nat. Prod. Res. 2020, 1-4. [CrossRef] [PubMed]

155. Ferhat, M.; Erol, E.; Beladjila, K.A.; Çetintaş, Y.; Duru, M.E.; Öztürk, M.; Kabouche, A.; Kabouche, Z. Antioxidant, anticholinesterase and antibacterial activities of Stachys guyoniana and Mentha aquatica. Pharm. Biol. 2016, 55, 324-329. [CrossRef] [PubMed]

156. Grigorakis, S.; Makris, D.P. Characterisation of polyphenol-containing extracts from Stachys mucronata and evaluation of their antiradical activity. Medicines 2018, 5, 14. [CrossRef]

157. Slapšytè, G.; Dedonytė, V.; Adomėnienè, A.; Lazutka, J.R.; Kazlauskaitė, J.; Ragažinskienė, O.; Venskutonis, P.R. Genotoxic properties of Betonica officinalis, Gratiola officinalis, Vincetoxicum luteum and Vincetoxicum hirundinaria extracts. Food Chem. Toxicol. 2019, 134, 110815. [CrossRef]

158. Kokhdan, E.P.; Ahmadi, K.; Sadeghi, H.; Sadeghi, H.; Dadgary, F.; Danaei, N.; Aghamaali, M.R. Hepatoprotective effect of Stachys pilifera ethanol extract in carbon tetrachloride-induce hepatotoxicity in rats. Pharm. Biol. 2017, 55, 1389-1393. [CrossRef]

159. Mansourian, M.; Mirzaei, A.; Azarmehr, N.; Vakilpour, H.; Kokhdan, E.P.; Doustimotlagh, A.H. Hepatoprotective and antioxidant activity of hydroalcoholic extract of Stachys pilifera. Benth on acetaminophen-induced liver toxicity in male rats. Heliyon 2019, 5, e03029. [CrossRef]

160. Hwang, J.Y.; Yadav, A.K.; Jang, B.C.; Kim, Y.C. Antioxidant and cytoprotective effects of Stachys riederi var. japonica ethanol extract on UVA-irradiated human dermal fibroblasts. Int. J. Mol. Med. 2019, 43, 1497-1504.

161. Lee, J.K.; Lee, J.; Kim, Y.-K.; Lee, Y.; Ha, J.-H. Stachys sieboldii Miq. root attenuates weight gain and dyslipidemia in rats on a high-fat and high-cholesterol diet. Nutrients 2020, 12, 2063. [CrossRef]

162. Ravichandran, V.A.; Kim, M.; Han, S.K.; Cha, Y. Stachys sieboldii extract supplementation attenuates memory deficits by modulating BDNF-CREB and its downstream molecules, in animal models of memory impairment. Nutrients 2018, 10, 917. [CrossRef]

163. Rahzani, K.; Malekirad, A.A.; Zeraatpishe, A.; Hosseini, N.; Seify, S.M.R.; Abdollahi, M. Anti-oxidative stress activity of Stachys lavandulifolia aqueous extract in human. Cell J. 2013, 14, 314-317. [PubMed]

164. Jalilian, N.; Modarresi, M.; Rezaei, M.; Ghaderi, L.; Bozorgmanesh, M. Phytotherapeutic management of polycystic ovary syndrome: Role of aerial parts of wood betony (Stachys lavandulifolia). Phytother. Res. 2013, 27, 1708-1713. [CrossRef] [PubMed]

165. Monji, F.; Hashemian, F.; Surmaghi, M.-H.S.; Mohammadyari, F.; Ghiyaei, S.; Soltanmohammadi, A. Therapeutic effects of standardized formulation of Stachys lavandulifolia Vahl on primary dysmenorrhea: A randomized, double-blind, crossover, placebo-controlled pilot study. J. Altern. Complement. Med. 2018, 24, 1092-1098. [CrossRef] [PubMed]

166. Ashtiani, A.R.; Jadidi, A.; Hezave, A.; Safarabadi, M.; Pour, S.A.; Ghassami, K.; Mohammadbeigi, A. An analgesic effect of Stachys lavandulifolia in patients with migraine: A double-blind randomised clinical trial study. Adv. Hum. Biol. 2019, 9, 76. [CrossRef]

167. Jafarzadeh, L.; Rafieian-Kopaei, M.; Samani, R.A.; Asgari, A. The effect of hydroalcoholic extract of Stachys lavandulifolia Vahl on pregnant mice. EXCLI J. 2012, 11, 357-362.

168. Taghikhani, M.; Nasri, H.; Asgari, A.; Afrough, H.; Namjoo, A.; Ansari-Samani, R.; Shahinfard, N.; Rafieian-Kopaei, M. The renal toxicity of hydroalcoholic extract of Stachys lavandulifolia Vahl in Wistar rats. Life Sci. J. 2012, 9, 3025-3031.

169. Modarresi, M.; Hosseinzadeh, L.; Nematy, N.; Siavash-Haghighi, Z.; Ghanbari, K. Acute and subchronic toxicological evaluation of Stachys lavandulifolia aqueous extract in Wistar rats. Res. Pharm. Sci. 2015, 9, 165. 
(C) 2020 by the authors. Licensee MDPI, Basel, Switzerland. This article is an open access article distributed under the terms and conditions of the Creative Commons Attribution (CC BY) license (http://creativecommons.org/licenses/by/4.0/). 